\title{
APPROXIMATING QUASI-STATIONARY DISTRIBUTIONS WITH INTERACTING REINFORCED RANDOM WALKS
}

\author{
Amarjit Budhiraja, Nicolas Fraiman*(1) And Adam Waterbury
}

\begin{abstract}
We propose two numerical schemes for approximating quasi-stationary distributions (QSD) of finite state Markov chains with absorbing states. Both schemes are described in terms of certain interacting chains in which the interaction is given in terms of the total time occupation measure of all particles in the system and has the impact of reinforcing transitions, in an appropriate fashion, to states where the collection of particles has spent more time. The schemes can be viewed as combining the key features of the two basic simulation-based methods for approximating QSD originating from the works of Fleming and Viot (1979) and Aldous, Flannery and Palacios (1998), respectively. The key difference between the two schemes studied here is that in the first method one starts with $a(n)$ particles at time 0 and number of particles stays constant over time whereas in the second method we start with one particle and at most one particle is added at each time instant in such a manner that there are $a(n)$ particles at time $n$. We prove almost sure convergence to the unique QSD and establish Central Limit Theorems for the two schemes under the key assumption that $a(n)=o(n)$. When $a(n) \sim n$, the fluctuation behavior is expected to be non-standard. Some exploratory numerical results are presented to illustrate the performance of the two approximation schemes.
\end{abstract}

Mathematics Subject Classification. 60J10, 34F05, 60F10, 92D25.

Received October 20, 2020. Accepted December 17, 2021.

\section{INTRODUCTION}

Markov processes with absorbing states occur frequently in epidemiology [2], statistical physics [33], and population biology [29]. Quasi-stationary distributions (QSD) are the basic mathematical object used to describe the long time behavior of such Markov processes on non-absorption events. Just as stationary distributions of ergodic Markov processes make the law of the Markov process, initialized at that distribution, invariant at all times, quasi-stationary distributions are probability measures that leave the conditional law of the Markov process, on the event of non-absorption, invariant. QSD have been widely studied since the pioneering work of Kolmogorov [26], Yaglom [36] and Sevastyanov [32], cf. [14, 29, 30]. Numerical computation of QSD is an important problem and the goal of this work is to investigate two related approximation schemes for QSD of finite state Markov chains. Specifically, we consider the following setting.

Let $\Delta$ denote a finite set and consider a nonempty subset $\partial \Delta \subset \Delta$. Let $\Delta^{o} \doteq \Delta \backslash \partial \Delta$ and assume that $\Delta^{o}$ is nonempty. Let $\left\{Y_{n}\right\}$ be a Markov chain taking values in $\Delta$ with transition probability kernel $\left\{P_{x, y}\right\}_{x, y \in \Delta}$. We

Keywords and phrases: Quasi-stationary distributions, stochastic approximation, interacting particles, central limit theorem, reinforced random walks, self-interaction, Fleming-Viot particle approximations.

Department of Statistics and Operations Research, University of North Carolina at Chapel Hill. Chapel Hill, NC 27599, USA.

* Corresponding author: fraiman@email.unc.edu 
denote by $\mathbf{P}_{\nu}$ the probability measure under which $\left\{Y_{n}\right\}$ has initial distribution $\nu$, namely $\mathbf{P}_{\nu}\left(Y_{0} \in A\right)=\nu(A)$. If $\nu=\delta_{x}$ for some $x \in \Delta$, we write $\mathbf{P}_{x}$ instead of $\mathbf{P}_{\nu}$. We assume that $\left\{Y_{n}\right\}$ is absorbed upon entering $\partial \Delta$. In particular, for each $x \in \partial \Delta$,

$$
\mathbf{P}_{x}\left(Y_{1} \in \Delta^{o}\right)=0
$$

Without loss of generality, we assume that $\partial \Delta$ consists of a single point which we denote by 0 . Note that

$$
\mathbf{P}_{x}\left(Y_{1}=y\right)=P_{x, y} \quad \text { for } x, y \in \Delta .
$$

A probability measure $\mu$ on $\Delta^{o}$ is a quasi-stationary distribution (QSD) for the chain $\left\{Y_{n}\right\}$ if

$$
\mathbf{P}_{\mu}\left(Y_{n}=x \mid Y_{n} \in \Delta^{o}\right)=\mu(x) \text {, for all } x \in \Delta^{o} \text { and } n \in \mathbb{N} \text {. }
$$

A set $S \subset \Delta$ is called an irreducible class of the Markov chain if for each $x, y \in S$, there is some $n \in \mathbb{N}_{0}$ such that $\mathbf{P}_{x}\left(Y_{n}=y\right)>0$. We assume that $\Delta^{o}$ is an irreducible class of the Markov chain and that the Markov chain can reach $\partial \Delta$ from $\Delta^{o}$. The latter property means that $P_{z, 0}>0$ for some $z \in \Delta^{o}$. Under this irreducibility assumption on the chain it follows from Perron-Frobenius theory that there is a unique QSD for $\left\{Y_{n}\right\}$ which we denote by $\theta_{*}$; see ([14], Chap. 3). This probability measure on $\Delta^{o}$ can be characterized as the normalized left eigenvector of the substochastic matrix $\left\{P_{x, y}\right\}_{x, y \in \Delta^{\circ}}$ associated with some eigenvalue $\lambda \in(0,1)$. In particular, unlike invariant distributions for Markov processes, the QSD is characterized as a solution of a nonlinear equation and thus presents harder numerical challenges. In general, numerical linear algebra methods become difficult when the underlying transition probability matrix is large or ill-conditioned. Thus, it is natural to explore simulation-based approaches.

There have been two main simulation-based approaches for approximating QSD. These approaches originate from the works of Fleming and Viot [20] and Aldous et al. [1], respectively. In numerical schemes based on the ideas of Fleming and Viot (see $[12,16]$ ), one considers a collection of particles evolving independently according to the Markov chain with transition probability kernel $\left\{P_{x, y}\right\}$, and whenever a particle is absorbed it jumps instantly to the position of another particle selected at random. It is known that as both time and the number of particles tend to infinity, the empirical measure of the current positions of the particles converges almost surely to the unique QSD $\theta_{*}[6,16,34]$. The method of Aldous et al. (see $[6,10]$ ) approximates the QSD with the time occupation measure of a single particle that evolves according to the transition kernel $\left\{P_{x, y}\right\}$ between visits to 0 , and when it hits 0 it jumps to a previously visited position with probability proportional to the time the chain spent at that position.

There has been substantial recent progress in analyzing the convergence rates of these algorithms. Cérou et al. [13] proved a Central Limit Theorem (CLT) for the law of Fleming-Viot particle systems at a given fixed time under very general assumptions. Lelievre et al. [28] obtained an infinite-time version in the setting of finite space Markov chains, extending the ideas of Del Moral and Miclo [15]. For the Aldous, Flannery and Palacios scheme, Benaïm and Cloez [6] and, independently, Blanchet, Glynn and Zheng [10] proved a Central Limit Theorem, see also [17, 18].

The convergence properties of the method of Aldous et al. have recently been studied in other contexts as well. Benaïm et al. [7] established the convergence of such methods in compact state space, and Benaïm et al. [5] and Wang et al. [35] proved similar results for diffusions with soft and hard killing respectively.

Each of the approximation methods discussed above has benefits and shortcomings. Approximating with several particles helps the approximation better explore the space, particularly when the Markov process has metastable states where a scheme using a single particle can get stuck in place for long periods of time. On the other hand, as the number of particles approach infinity, a Fleming-Viot approximation approaches the conditional law of the Markov chain (conditioned on non-extinction) at some finite-time instant rather than the QSD, and thus in order to obtain a good approximation for the QSD one needs to run the algorithm over long time periods. This can be computationally expensive and numerical experiments (see Sect. 7) suggest 
that, with equivalent number of particle moves, a single particle reinforced random walk scheme of Aldous et al. performs better than a Fleming-Viot type scheme. This trade-off between the exploration of state space through multiple particles and the reinforcement of particle transition probabilities based on the time occupation measure motivates the present work, which studies two algorithms that combine desirable features of both approximation schemes.

The two schemes that we study consider a collection of particles that, unlike Fleming-Viot approximations in which interactions occur through the current particle states, are governed by interactions with the time occupancy measures of all particles. Specifically, when a particle is absorbed, it instantly jumps to a state with probability proportional to the total time spent at that position by all the particles in the collection. The main difference between the two schemes considered in this work is that in the first scheme we start with $a(n)$ particles at time 0 and the number of particles stays constant over time, whereas in the second scheme we add one particle at a time at some fixed rate so that there are $a(n)$ particles at time instant $n$. The approximation to the QSD is given by the combined (and suitably normalized) time occupation measure of all particles in the system. Our main results, Theorems 1.2, 1.3, 1.4, and 1.5 provide a.s. convergence to the QSD (i.e. strong law of large numbers) and central limit theorems for the two schemes. In Section 7 we present some exploratory numerical results on the performance of the two schemes and its comparison with the Fleming-Viot and Aldous et al. methods. The approach to the mathematical analysis of the two schemes is inspired by the methods used in [6], for the study of the Aldous et al. scheme based on the path of a single particle, and draws from techniques for establishing central limit results for general stochastic approximation schemes developed by Delyon [19] and Fort [21].

The theory of stochastic approximations (SA) has a long history, starting from the works of Robbins and Monro [31], and Kiefer and Wolfowitz [25]. Since then, it has found many applications and has developed into a thriving area of research $[9,11,27]$. In a typical stochastic approximation scheme one constructs a discrete time stochastic process whose continuous time interpolation over suitably slow decreasing time steps approaches the fixed point of a deterministic ordinary differential equation (ODE) as the continuous time parameter $t$ approaches infinity. One of the key differences, from this standard setting, in the analysis of our first scheme presented in Sections 2-4, is that instead of a single stochastic approximation sequence, one needs to study an array, indexed by $n$, of sequences such that for each $n$ the sequence can be viewed as a SA algorithm targeting the QSD as the number of steps increase. Our first result, Theorem 1.2, provides a strong law of large numbers for this array as $n$ and the number of time steps become large. This result also provides an almost sure upper bound on the rate of convergence which plays a crucial role later in the proof of the central limit theorem in Theorem 1.3. In order to establish a suitable rate of convergence, we introduce the notion of pseudo-trajectory sequences (see Def. 2.1), which is inspired by the ideas of asymptotic pseudo-trajectories, first introduced in [8] (see also $[3,6]$ ), and is well-suited for array-type schemes such as those considered here.

In Theorem 1.3 we establish a central limit theorem for the array by considering the $n$-th sequence run for $n$ time steps. The proof uses several ideas from Section 4 of [21]. In that work, the author considers a general SA algorithm which covers settings such as that of a controlled Markov chain that evolves, conditional on the past history of the system, according to a stochastic kernel depending on the current approximation. The proofs of [21] do not easily extend to array settings of the form considered in the current work and it turns out that the rate of convergence in Theorem 1.2 is key to suitably controlling the error arrays in the martingale decomposition of the SA sequences. One of the key requirements in the proofs is that $a(n)=o(n)$. Indeed, when $a(n) \sim n$, the errors due to the finite-time behavior of the collection of particles can accumulate and the fluctuation properties under the natural central limit scaling can be somewhat non-standard, see Remark 1.7 for a discussion of this point.

While in this work our focus is on approximating the QSD of a finite state Markov chain, the approach used to prove Theorems 1.2 and 1.3 is more generally applicable. In particular, the notion of a pseudo-trajectory sequence introduced in Definition 2.1 should be useful for obtaining bounds on the rate of convergence and establishing central limit theorems for other types of SA arrays.

The second numerical scheme is studied in Section 5. In this method the approximation is initialized with a single particle and as time progresses particles are added to the system. At each step at most one particle 
is added and the number of particles at time $n$ is denoted by $a(n)$. Once more, the combined time occupation measure of all particles is used to approximate the QSD and to replace particles that get absorbed. Since the number of particles changes over time, the analysis of error terms and the covariance structure gets more involved. In order to keep the presentation simple, here we restrict attention to the case where $a(n) \sim n^{\zeta}$ for some $\zeta \in(0,1)$. In Theorem 1.4 we prove a.s. convergence of the approximation to the QSD and in Theorem 1.5 we provide a central limit theorem for this approximation scheme.

One of the challenges in constructing stochastic approximation schemes, with provable central limit fluctuations, for approximating QSD using a large number of particles is to carefully analyze the contribution to the variance and bias due to the finite-time behavior of the dynamics and to suitably calibrate the weights given to particle states as time increases. Specifically, for the two algorithms studied in the current work, we find that in comparison to the single particle SA schemes studied in $[6,10]$, one needs to place higher weights on particle states at later time instants in order to suitably counterbalance the variability due to the finite-time behavior of the chains. This point is discussed further in Remark 1.6, however a precise understanding of relationships between size of SA arrays and time step sizes, for central limit results to hold, remains to be fully developed. Finally, we remark that in this work we consider SA arrays and sequences with time steps of order $1 / n$. Convergence and fluctuation results for interacting particle schemes with more general time steps satisfying appropriate decay conditions will be a topic for future study.

We now comment on some contributions of this work. One of the main challenges is that unlike previous works $[6,10]$ which require an analysis of a single SA sequence, here one needs to study asymptotic behavior of an SA array $\left\{\theta_{k}^{n}, n \in \mathbb{N}, k \in \mathbb{N}_{0}\right\}$. Specifically we need to understand the behavior as the time parameter $(k)$ and the number of particles $(n)$ increase simultaneously. The array feature of our SA scheme requires several new ideas. One of these is the extension of the notion of asymptotic pseudo-trajectories introduced in [8] to pseudo-trajectory sequences (see Def. 2.1). These sequences play a key role in providing a.s. upper bounds on rates of convergence of SA arrays that are uniform in a suitable sense, which in turn are used in a crucial way in the proof of the central limit in Theorem 1.3. Indeed, we expect that ideas based on pseudo-trajectory sequences will be useful for proving rate-of-convergence results and central limit theorems for a broader class of stochastic approximation arrays and will be of interest to researchers in stochastic approximation methods for other application areas. Proofs of results that provide rates of convergence bounds are inspired by [6], however there is one key step where new ideas are needed to control certain martingale difference terms in a uniform manner as time and number of particles increase simultaneously (see Lems. 2.4 and 2.9). Theorem 1.3 gives a novel form of CLT which describes fluctuations of the estimates as the number of particles and time increase simultaneously and we expect such fluctuation results to be of interest for other types of SA arrays. Proof strategy for the CLT is strongly influenced by Delyon [19] and Fort [21] but it also has one key new ingredient of leveraging the a.s. rates of convergence to show that various types of error terms decay sufficiently fast (see e.g. Prop. 4.1 and Cor. 4.2). These convergence rates are also key in estimating (in a uniform manner) the conditional covariances of certain noise arrays (see Lem. 4.5). The analysis reveals a subtle balance between the number of particles and relative contributions of early versus later time steps that is needed in the CLT proof. These points are discussed in detail in Remarks 1.6 and 1.7.

We now describe the two schemes in some detail.

\subsection{Description of the algorithms}

We denote by $\mathcal{P}\left(\Delta^{o}\right)$ the space of probability measures on $\Delta^{o}$. Letting $d \doteq\left|\Delta^{o}\right|, \mathcal{P}\left(\Delta^{o}\right)$ can be identified with the $(d-1)$-dimensional simplex

$$
\mathcal{S} \doteq\left\{x \in \mathbb{R}_{+}^{d}: \sum_{i=1}^{d} x_{i}=1\right\}
$$


For notational convenience, elements of $\Delta^{o}$ will be labeled as $\{1,2, \ldots, d\}$. For each $\nu \in \mathcal{P}\left(\Delta^{o}\right)$, we consider a transition probability kernel $K[\nu]$ on $\Delta^{o}$ given by

$$
K[\nu]_{x, y} \doteq P_{x, y}+P_{x, 0} \nu(y) \quad \text { for } x, y \in \Delta^{o} .
$$

For each $\nu \in \mathcal{P}\left(\Delta^{o}\right)$, the Markov chain associated with the transition probability kernel $K[\nu]$ is irreducible, and we denote the corresponding unique invariant distribution by $\pi(\nu)$. Define

$$
h(\nu) \doteq \pi(\nu)-\nu \quad \text { for } \nu \in \mathcal{P}\left(\Delta^{o}\right) .
$$

It is well known that $h: \mathcal{S} \rightarrow T \mathcal{S} \doteq\left\{x \in \mathbb{R}^{d}: \sum_{i=1}^{d} x_{i}=0\right\}$ is a smooth function and the Jacobian matrix $\nabla h\left(\theta_{*}\right)$ is a Hurwitz matrix, in particular there is some $L>0$ such that the eigenvalues of $\nabla h\left(\theta_{*}\right)$ have their real parts bounded above by $-L$; see Corollary 2.3 of [6].

The approximation algorithms described below are given in terms of a certain step size sequence denoted by $\left\{\gamma_{n}\right\}_{n=1}^{\infty}$, and we assume that for some $\gamma_{*}>0$, we have

$$
\gamma_{k+1} \doteq \frac{\gamma_{*}}{k+N_{*}}, k \in \mathbb{N}_{0}
$$

where $N_{*}=\left\lfloor\gamma_{*}\right\rfloor+1$. This choice of $N_{*}$ ensures that $\gamma_{k+1} \leq 1$ for each $k \in \mathbb{N}_{0}$, which in turn guarantees that the two algorithms' estimates for the QSD are probability measures. In general, $N_{*}$ can be taken to be any positive real such that $\gamma_{k+1} \leq 1$ for each $k \in \mathbb{N}_{0}$. Let $\{a(n)\}_{n \in \mathbb{N}}$ be a sequence of positive integers increasing to $\infty$.

Algorithm I. For fixed $x_{0} \in \Delta^{o}$, we consider a collection $\left\{X_{k}^{i, n}\right\}_{1 \leq i \leq a(n), n \in \mathbb{N}, k \in \mathbb{N}_{0}}$ of $\Delta^{o}$-valued random variables, an array $\left\{\theta_{k}^{n}\right\}_{n \in \mathbb{N}, k \in \mathbb{N}_{0}}$ of $\mathcal{P}\left(\Delta^{o}\right)$-valued random measures, and a collection $\left\{\mathcal{F}_{k}^{n}\right\}_{n \in \mathbb{N}, k \in \mathbb{N}_{0}}$ of $\sigma$-fields given on some probability space $(\Omega, \mathcal{F}, \mathbf{P})$, defined recursively as follows. For $n \in \mathbb{N}$ and $k=0$, let

$$
X_{0}^{i, n} \doteq x_{0}, \quad 1 \leq i \leq a(n), \quad \mathcal{F}_{0}^{n} \doteq\{\emptyset, \Omega\} \quad \text { and } \quad \theta_{0}^{n} \doteq \delta_{x_{0}}
$$

Having defined the above random variables and $\sigma$-fields for some $k \in \mathbb{N}_{0}$ and all $n \in \mathbb{N}$, define, for each $n \in \mathbb{N}$ and $1 \leq i \leq a(n)$

$$
\mathbf{P}\left(X_{k+1}^{1, n}=y_{1}, \ldots, X_{k+1}^{a(n), n}=y_{a(n)} \mid \mathcal{F}_{k}^{n}\right)=\prod_{i=1}^{a(n)} K\left[\theta_{k}^{n}\right]_{x_{i}, y_{i}} .
$$

on the set $\left\{X_{k}^{1, n}=x_{1}, \ldots, X_{k}^{a(n), n}=x_{a(n)}\right\}$. The filtration is extended as

$$
\mathcal{F}_{k+1}^{n} \doteq \mathcal{F}_{k}^{n} \vee \sigma\left(X_{k+1}^{1, n}, \ldots, X_{k+1}^{a(n), n}\right)
$$

and the new estimate of the QSD is given by

$$
\theta_{k+1}^{n} \doteq\left(1-\gamma_{k+1}\right) \theta_{k}^{n}+\gamma_{k+1} \frac{1}{a(n)} \sum_{i=1}^{a(n)} \delta_{X_{k+1}^{i, n}}
$$


We are interested in the asymptotic behavior of $\theta_{n} \doteq \theta_{n}^{n}$. In order to write $\left\{\theta_{k}^{n}\right\}_{n=1}^{\infty}$ as a stochastic approximation (SA) algorithm, for $1 \leq i \leq a(n)$ and $k \in \mathbb{N}_{0}$, let

$$
\epsilon_{k+1}^{n} \doteq \frac{1}{a(n)} \sum_{i=1}^{a(n)} \epsilon_{k+1}^{i, n} \quad \text { where } \quad \epsilon_{k+1}^{i, n} \doteq \delta_{X_{k+1}^{i, n}}-\pi\left(\theta_{k}^{n}\right) .
$$

Then the evolution for the QSD approximation $\theta_{k}^{n}$ from (1.4) can be rewritten as

$$
\theta_{k+1}^{n}=\theta_{k}^{n}+\gamma_{k+1}\left(h\left(\theta_{k}^{n}\right)+\epsilon_{k+1}^{n}\right) .
$$

Note that when $\gamma_{*}=1$ and $N_{*}=1, \theta_{k}^{n}$ is simply the empirical measure of the previous $(k+1) a(n)$ states of the $a(n)$ particles in the system.

Algorithm II. In order to distinguish from the notation used for the first scheme, we will use bold symbols to denote some key quantities with slightly different definitions than those in the definition of the first algorithm. In this method, rather than starting with $a(n)$ particles, we will start with 1 particle at time 0 and add particles over time. This algorithm is therefore described by a single sequence of random variables rather than by an array. In particular $a(n)$ will denote the number of particles at the $n$-th time step rather than the number of particles in the $n$-th sequence in the array. Here $\{a(n)\}$ is a non-decreasing sequence of integers satisfying the following:

(A) $a(0)=1$.

(B) For each $n \in \mathbb{N}, a(n+1)-a(n) \leq 1$.

(C) The number of particles at instant $n$ is $a(n)$ and there is some $\zeta \in(0,1)$ such that the $n$-th particle is added at time step $b(n)=\left\lfloor n^{1 / \zeta}\right\rfloor$.

The above properties in particular say that $a(n) \sim n^{\zeta}$ and the sequence $\{b(n)\}$ satisfies $b(1) \doteq 0$, and

$$
b(n) \doteq \inf \{m>b(n-1): a(m)=a(n-1)+1\} .
$$

The above conditions for $\{a(n)\}$ are satisfied, for example, when $a(n) \doteq \max \left\{1,\left\lfloor n^{\zeta}\right\rfloor\right\}$ for some $\zeta \in(0,1)$. Furthermore, as noted in Remark 1.1, condition (B) above can be replaced by the assumption of uniform boundedness of the increments $a(n+1)-a(n)$. We will also need a $\{1, \ldots, a(n)\}$ valued random variable $\iota_{n}$ which will tell us where to add the new particle at time instant $n+1$ if $a(n+1)=a(n)+1$. The precise manner in which this particle is added is not important and one can use an arbitrary non-anticipative rule for doing so. More precisely, the scheme is given as follows.

Consider a collection $\left\{\boldsymbol{X}_{n}^{i}\right\}_{1 \leq i \leq a(n+1), n \in \mathbb{N}_{0}}$ of $\Delta^{o}$-valued random variables, a sequence $\left\{\iota_{n}\right\}_{n \in \mathbb{N}_{0}}$ of random variables with $\iota_{n}$ taking values in $\{1, \ldots, a(n)\}$, a sequence $\left\{\boldsymbol{\theta}_{n}\right\}_{n \in \mathbb{N}_{0}}$ of $\mathcal{P}\left(\Delta^{o}\right)$-valued random measures, and a sequence $\left\{\mathcal{F}_{n}\right\}_{n \in \mathbb{N}_{0}}$ of $\sigma$-fields given on some probability space $(\Omega, \mathcal{F}, \mathbf{P})$, recursively defined as follows. We let

$$
\boldsymbol{X}_{0}^{1} \doteq x_{0}, \quad \iota_{0} \doteq 1, \quad \mathcal{F}_{0} \doteq\{\emptyset, \Omega\} \quad \text { and } \quad \boldsymbol{\theta}_{0} \doteq \delta_{x_{0}}
$$

Note $a(0)=a(1)=1$. We let $\iota_{1} \doteq 1$. Having defined $\left\{\boldsymbol{X}_{n}^{i}\right\}_{1 \leq i \leq a(n+1)}, \boldsymbol{\theta}_{n}, \iota_{n+1}$ and $\mathcal{F}_{n}$, define the elements for the next step as follows:

- Conditioned on $\mathcal{F}_{n}$, particles evolve according to the kernel $K\left[\boldsymbol{\theta}_{n}\right]$ independently. In particular, if no branching occurs, namely $a(n+2)=a(n+1)$, then

$$
\mathbf{P}\left(\boldsymbol{X}_{n+1}^{1}=y_{1}, \ldots, \boldsymbol{X}_{n+1}^{a(n+2)}=y_{a(n+2)} \mid \mathcal{F}_{n}\right)=\prod_{i=1}^{a(n+1)} K\left[\boldsymbol{\theta}_{n}\right]_{x_{i}, y_{i}}
$$


on the set $\left\{\boldsymbol{X}_{n}^{1}=x_{1}, \ldots, \boldsymbol{X}_{n}^{a(n+1)}=x_{a(n+1)}\right\}$. On the other hand, if a branching event occurs, i.e. $a(n+2)=$ $a(n+1)+1$, on the set $\left\{\boldsymbol{X}_{n}^{1}=x_{1}, \ldots, \boldsymbol{X}_{n}^{a(n+1)}=x_{a(n+1)}\right.$ and $\left.\iota_{n+1}=\ell\right\}$, the particle with index $\ell$ will replicate, the new particle be given the index $a(n+2)$, and

$$
\mathbf{P}\left(\boldsymbol{X}_{n+1}^{1}=y_{1}, \ldots, \boldsymbol{X}_{n+1}^{a(n+2)}=y_{a(n+2)} \mid \mathcal{F}_{n}\right)=\left(\prod_{i=1}^{a(n+1)} K\left[\boldsymbol{\theta}_{n}\right]_{x_{i}, y_{i}}\right) K\left[\boldsymbol{\theta}_{n}\right]_{x_{\ell}, y_{a(n+2)}} .
$$

- With $\mathcal{G}_{n+1} \doteq \mathcal{F}_{n} \vee \sigma\left\{\boldsymbol{X}_{n+1}^{1}, \ldots, \boldsymbol{X}_{n+1}^{a(n+2)}\right\}$ and $\mathcal{H}_{n+1}$ an arbitrary $\sigma$-field independent of $\mathcal{G}_{n+1}$, let $\iota_{n+2}$ be an arbitrary $\mathcal{G}_{n+1} \vee \mathcal{H}_{n+1}$ measurable random variable with values in $\{1, \ldots, a(n+2)\}$.

- Let $\mathcal{F}_{n+1}=\mathcal{F}_{n} \vee \sigma\left(\boldsymbol{X}_{n+1}^{1} \ldots, \boldsymbol{X}_{n+1}^{a(n+2)}\right) \vee \sigma\left(\iota_{n+2}\right)$.

- Finally, let the new QSD estimate be

$$
\boldsymbol{\theta}_{n+1} \doteq\left(1-\gamma_{n+1}\right) \boldsymbol{\theta}_{n}+\gamma_{n+1} \frac{1}{a(n+1)} \sum_{i=1}^{a(n+1)} \delta_{\boldsymbol{X}_{n+1}^{i}} .
$$

Note that by construction, $\boldsymbol{\theta}_{n},\left\{\boldsymbol{X}_{n}^{i}\right\}_{1 \leq i \leq a(n+1)}$, and $\iota_{n+1}$ are $\mathcal{F}_{n}$ measurable for all $n \in \mathbb{N}_{0}$. Also note that $\iota_{n+1}$ plays a role in the definition of the measure $\boldsymbol{\theta}_{n+2}$ only when $a(n+2)=a(n+1)+1$.

In order to write $\boldsymbol{\theta}_{n}$ as a SA algorithm, we define, for $1 \leq i \leq a(n+1)$ and $n \in \mathbb{N}_{0}$,

$$
\boldsymbol{\epsilon}_{n+1} \doteq \frac{1}{a(n+1)} \sum_{i=1}^{a(n+1)} \boldsymbol{\epsilon}_{n+1}^{i} \quad \text { where } \quad \boldsymbol{\epsilon}_{n+1}^{i} \doteq \delta_{\boldsymbol{X}_{n+1}^{i}}-\pi\left(\boldsymbol{\theta}_{n}\right)
$$

Then the evolution equation in (1.8) can be rewritten as

$$
\boldsymbol{\theta}_{n+1}=\boldsymbol{\theta}_{n}+\gamma_{n+1}\left(h\left(\boldsymbol{\theta}_{n}\right)+\boldsymbol{\epsilon}_{n+1}\right) .
$$

Remark 1.1. Condition (B) describing the sequence $\{a(n)\}$ can be replaced by the following assumption: there is some $c \in[1, \infty)$ such that for each $n \in \mathbb{N}, a(n+1)-a(n) \leq c$. Our restriction to the case when $c=1$ is done for notational convenience, and the case when $c \geq 1$ can be handled similarly.

\subsection{Statement of results}

We first describe the results for Algorithm I, namely the algorithm given by (1.6). The following theorem proves that the approximation scheme converges a.s. to the unique QSD $\theta_{*}$ and provides an a.s. upper bound on the rate at which $\left\{\theta_{k}^{n}\right\}$ converges to $\theta_{*}$.

Theorem 1.2. As $n \rightarrow \infty, \theta_{n}^{n} \rightarrow \theta_{*}$ almost surely. Furthermore, for each $p \in(0,1)$, there is a $\beta>0$, such that for $\mathbf{P}$-a.e. $\omega$, there is a $n_{0} \equiv n_{0}(\omega) \in \mathbb{N}$ such that for all $n \geq n_{0}$ and $n^{p} \leq k \leq n$,

$$
\left\|\theta_{k}^{n}-\theta_{*}\right\| \leq k^{-\beta} .
$$

Note that the constant $p$ appearing in Theorem 1.2 determines the length of the time interval (for each $n$ ) over which the result provides an a.s. upper bound on the algorithm's rate of convergence. In particular, the smaller $p$ is, the longer the interval $\left[n^{p}, n\right]$ is. Therefore, by choosing a smaller value of $p$, one can ensure some additional uniformity (in time) of the rate of convergence. However, this has some trade-offs, as the constant $\beta$ determining the rate of convergence depends on $p$ as well, and, in general, may decrease as $p$ decreases. Note that, since the intervals $\left[n^{p}, n\right]$ decrease with $p$, for any $p^{*} \in(0,1)$, the estimate in the above theorem holds for all $p \in\left(p^{*}, 1\right]$ with the same choice of $\beta$ and $n_{0}$. 
Theorem 1.2 is proved in Section 2. The next theorem provides a central limit theorem for the sequence $\left\{\theta_{n}^{n}\right\}$. Define the sequence $\left\{\sigma_{n}\right\}_{n=1}^{\infty}$ by

$$
\sigma_{n} \doteq \sqrt{a(n) / \gamma_{n}}
$$

This sequence will give the scaling factor in the CLT. The covariance matrix for the limiting Gaussian distribution is given in terms of a nonnegative definite matrix $U_{*}$ which is introduced later in (3.2). For the CLT we will need additional conditions on the step sizes and the number of particles in the system. Recall from Section 1.1 that $L>0$ is a constant such that the eigenvalues of $\nabla h\left(\theta_{*}\right)$ have their real parts bounded above by $-L$. The additional conditions on $\gamma_{*}$ and $L$ required for Theorem 1.3 and Theorem 1.5 and their comparison with the conditions in [6] are discussed in Remark 1.6.

Theorem 1.3. Suppose that $a(n) / n \rightarrow 0$ as $n \rightarrow \infty$ and $\gamma_{*}>L^{-1}$. Then, as $n \rightarrow \infty$,

$$
\sigma_{n}\left(\theta_{n}^{n}-\theta_{*}\right) \stackrel{\mathcal{L}}{\rightarrow} \mathcal{N}(0, V)
$$

where $V$ is the solution to the Lyapunov equation

$$
U_{*}+\nabla h\left(\theta_{*}\right) V+V \nabla h\left(\theta_{*}\right)^{T}+\gamma_{*}^{-1} V=0,
$$

$U_{*}$ is the nonnegative definite matrix given by (3.2), and $\stackrel{\mathcal{L}}{\rightarrow}$ denotes convergence in distribution.

Theorem 1.3 is proved in Section 4 by combining results from Sections 2 and 3. The Lyapunov equation (1.12) characterizes the asymptotic covariance of the algorithm's (scaled) fluctuations around the QSD. Such Lyapunov equations are frequently used to characterize the asymptotic covariance structure of stochastic approximation techniques such as those considered in this work (see e.g., [21], Thm. 2.1 and [19], Thm. 24). For fundamental results regarding the existence and uniqueness of solutions to such Lyapunov equations, see [24] and references therein.

The following are our main results for Algorithm II given by (1.10). The first result proves the a.s. convergence of the scheme. This time we don't provide convergence rates as it turns out that unlike the proof of Theorem 1.3, the proof of Theorem 1.5 does not require the use of convergence rates. Recall that, in order to distinguish from the quantities that appear in Algorithm I, we denote the corresponding quantities in boldface.

Theorem 1.4. As $n \rightarrow \infty, \boldsymbol{\theta}_{n} \rightarrow \theta_{*}$ almost surely.

Theorem 1.4 is proved in Section 5 .

Our final result gives a CLT for Algorithm II. Proof is given in Section 6 .

Theorem 1.5. Suppose that $a(n) / n \rightarrow 0$ as $n \rightarrow \infty$ and $\gamma_{*}>L^{-1}$. Then, as $n \rightarrow \infty$,

$$
\sigma_{n}\left(\boldsymbol{\theta}_{n}-\theta_{*}\right) \stackrel{\mathcal{L}}{\rightarrow} \mathcal{N}(0, \boldsymbol{V})
$$

where $V$ is the solution to the Lyapunov equation

$$
U_{*}+\nabla h\left(\theta_{*}\right) \boldsymbol{V}+\boldsymbol{V} \nabla h\left(\theta_{*}\right)^{T}+(1+\zeta) \gamma_{*}^{-1} \boldsymbol{V}=0
$$

and $U_{*}$ is the nonnegative definite matrix given by (3.2).

Remark 1.6. The condition $\gamma_{*}>L^{-1}$ is used in an important way in the proofs of CLT in Theorems 1.3 and 1.5. We note that the CLT for a single particle scheme given in [6] allows for any $\gamma_{*}>(2 L)^{-1}$. Thus, we find that for CLT results here we need larger step sizes than those allowed for the single particle scheme. Larger step sizes correspond to placing higher weights on particle states at later time instants. This need for 
suitably emphasizing later time points more arises in order to counterbalance the variability due to the large number of particles at any fixed time instant. The fact that a large number of particles at any fixed instant can qualitatively change the behavior of the fluctuations is further discussed in Remark 1.7.

Remark 1.7. Recall that for the CLT results we require that $a(n)=o(n)$. This condition is crucial in obtaining the estimates on the discrepancy array (resp. sequence) given in Lemma 4.3 (resp. Prop. 6.6). As noted in the Introduction, when $a(n) \sim n$ one expects nonstandard fluctuation behavior under the natural CLT scaling. To see this, consider the elementary setting of a collection of i.i.d. Markov chains. Specifically, let $\left\{X_{m}^{n}, m \in \mathbb{N}_{0}\right\}_{n \in \mathbb{N}}$ be a collection of i.i.d. irreducible Markov chains on $\Delta^{o}$ with transition probability kernel $K_{0}$ and stationary distribution $\theta_{*}$. For simplicity suppose that $X_{0}^{n}=x_{0}$ for all $n \in \mathbb{N}$, for some $x_{0} \in \Delta^{o}$. Define

$$
\theta_{m}^{n}=\frac{1}{m a(n)} \sum_{k=1}^{m} \sum_{i=1}^{a(n)}\left(\delta_{X_{k}^{i}}-\theta_{*}\right), \quad m, n \in \mathbb{N} .
$$

It is straightforward to show that if $a(n)=o(n)$, then, as $n \rightarrow \infty$,

$$
\sqrt{a(n) n}\left(\theta_{n}^{n}-\theta_{*}\right) \stackrel{\mathcal{L}}{\rightarrow} \mathcal{N}\left(0, U_{*}\right) .
$$

where $U_{*}$ is defined in a similar manner as in (3.2). However when $a(n) \sim a_{*} n$ for some $a_{*} \in(0, \infty)$, a different behavior emerges, and in fact the asymptotic mean of the scaled differences $\sqrt{a(n) n}\left(\theta_{n}^{n}-\theta_{*}\right)$ is nonzero as $n \rightarrow \infty$. In particular, one can easily see that

$$
\sqrt{a(n) n}\left(\theta_{n}^{n}-\theta_{*}\right) \stackrel{\mathcal{L}}{\rightarrow} \mathcal{N}\left(\alpha_{*}, U_{*}\right),
$$

where

$$
\alpha_{*} \doteq a_{*}\left[\left(K_{0} Q_{0}\right)_{x_{0}, \cdot}-\theta_{*}\left(K_{0} Q_{0}\right)\right], \quad \theta_{*}\left(K_{0} Q_{0}\right) \doteq \sum_{x \in \Delta^{o}}\left(K_{0} Q_{0}\right)_{x,}, \theta_{*}(x)
$$

where $Q_{0}$ is defined as in (1.15) on replacing on its right side $K[\nu]$ with $K_{0}$ and $\Pi(\nu)$ with the $d \times d$ matrix $\left[\theta_{*}, \theta_{*}, \cdots\right]^{T}$. For the stochastic approximation algorithms considered in this work, in order to study the limit behavior when $a(n) \sim n$ one will need to carefully analyze the limiting behavior of state dependency in the (appropriately scaled) discrepancy array/sequence, which describes the deviations of the linearized evolution from the underlying stochastic approximation algorithm (see discussion in Sect. 1.3) in order to identify the asymptotic 'drift' in the Gaussian limit. This study will be taken up elsewhere.

Remark 1.8. Since in Algorithm II one particle is added at a time and at time $k$ there are $a(k)$ particles, a more natural choice of the central limit scaling than $\sigma_{n}$ is given by the sequence

$$
\beta_{n} \doteq\left(\gamma_{n} \frac{1}{n} \sum_{k=1}^{n} \frac{1}{a(k)}\right)^{-1 / 2} .
$$

From Theorem 1.5 it follows immediately that

$$
\beta_{n}\left(\boldsymbol{\theta}_{n}-\theta_{*}\right) \stackrel{\mathcal{L}}{\rightarrow} \mathcal{N}(0, \tilde{\boldsymbol{V}}),
$$

where $\tilde{\boldsymbol{V}}$ is the unique solution to the Lyapunov equation

$$
(1-\zeta) U_{*}+(1+\zeta) \gamma_{*}^{-1} \tilde{\boldsymbol{V}}+\nabla h\left(\theta_{*}\right) \tilde{\boldsymbol{V}}+\tilde{\boldsymbol{V}} \nabla h\left(\theta_{*}\right)^{T}=0 .
$$


On the other hand, recall that for Algorithm I the central limit theorem takes the form

$$
\sigma_{n}\left(\theta_{n}^{n}-\theta_{*}\right) \stackrel{\mathcal{L}}{\rightarrow} \mathcal{N}(0, V)
$$

where $V$ is the solution to (1.12). The quantities $V$ and $\tilde{\boldsymbol{V}}$ can be viewed as the 'per-particle' asymptotic covariance matrices for the two numerical schemes.

\subsection{Decomposition and linearization}

One of the key ingredients in the proofs is the following explicit representation of the solution of Poisson's equation associated with the transition probability kernel $K[\cdot]$. For a proof, see Lemma 5.1 of [3].

Lemma 1.9. For each $\nu \in \mathcal{P}\left(\Delta^{o}\right)$, let $\Pi(\nu)$ be the $d \times d$ matrix with entries $\Pi(\nu)_{x, y}=\pi(\nu)_{y}$. Then for each $\nu \in \mathcal{P}\left(\Delta^{o}\right)$, the matrix

$$
Q[\nu] \doteq-\int_{0}^{\infty}[\exp (t(K[\nu]-I))-\Pi(\nu)] \mathrm{d} t
$$

is well-defined and the map $\nu \mapsto Q[\nu]$ is continuously differentiable. Furthermore,

$$
(I-K[\nu]) Q[\nu]=Q[\nu](I-K[\nu])=I-\Pi(\nu) .
$$

Using the above result, and following [3,6], we decompose the noise in Algorithm I given in (1.5) in the following manner: for each $n \in \mathbb{N}$ and $1 \leq i \leq a(n)$, write

$$
\begin{aligned}
\epsilon_{k+1}^{i, n} & =\delta_{X_{k+1}^{i, n}}-\pi\left(\theta_{k}^{n}\right)=\left(X_{k+1}^{i, n}\right)^{T}\left(I-\Pi\left(\theta_{k}^{n}\right)\right) \\
& =\left(X_{k+1}^{i, n}\right)^{T}\left(I-K\left[\theta_{k}^{n}\right]\right) Q\left[\theta_{k}^{n}\right]=Q\left[\theta_{k}^{n}\right]_{X_{k+1}^{i, n}, \cdot}-\left(K\left[\theta_{k}^{n}\right] Q\left[\theta_{k}^{n}\right]\right)_{X_{k+1}^{i, n},} .
\end{aligned}
$$

Then we can write $\epsilon_{k+1}^{i, n}=e_{k+1}^{i, n}+r_{k+1}^{i, n}$ where

$$
e_{k+1}^{i, n} \doteq Q\left[\theta_{k}^{n}\right]_{X_{k+1}^{i, n}, \cdot}-\left(K\left[\theta_{k}^{n}\right] Q\left[\theta_{k}^{n}\right]\right)_{X_{k}^{i, n},,}, r_{k+1}^{i, n} \doteq\left(K\left[\theta_{k}^{n}\right] Q\left[\theta_{k}^{n}\right]\right)_{X_{k}^{i, n}, \cdot}-\left(K\left[\theta_{k}^{n}\right] Q\left[\theta_{k}^{n}\right]\right)_{X_{k+1}^{i, n},}
$$

For each $n, k \in \mathbb{N}$, define the error and remainder arrays $\left\{e_{k}^{n}\right\}$ and $\left\{r_{k}^{n}\right\}$ by

$$
e_{k+1}^{n} \doteq \frac{1}{a(n)} \sum_{i=1}^{a(n)} e_{k+1}^{i, n}, \quad r_{k+1}^{n} \doteq \frac{1}{a(n)} \sum_{i=1}^{a(n)} r_{k+1}^{i, n} .
$$

Then the algorithm defined in (1.4) can be written as

$$
\theta_{k+1}^{n} \doteq \theta_{k}^{n}+\gamma_{k+1} h\left(\theta_{k}^{n}\right)+\gamma_{k+1} e_{k+1}^{n}+\gamma_{k+1} r_{k+1}^{n} .
$$

Along with the above evolution equation, it will be helpful to consider the linearized evolution array $\left\{\mu_{k}^{n}\right\}$ given by

$$
\mu_{0}^{n} \doteq 0 \quad \text { and } \quad \mu_{k+1}^{n} \doteq\left(I+\gamma_{k+1} \nabla h\left(\theta_{*}\right)\right) \mu_{k}^{n}+\gamma_{k+1} e_{k+1}^{n}
$$

and to study the discrepancy array $\left\{\rho_{k}^{n}\right\}$ given by

$$
\rho_{0}^{n} \doteq \theta_{0}^{n}-\theta_{*} \quad \text { and } \quad \rho_{k}^{n} \doteq\left(\theta_{k}^{n}-\theta_{*}\right)-\mu_{k}^{n} .
$$


Note that $\theta_{k}^{n}-\theta_{*}=\mu_{k}^{n}+\rho_{k}^{n}$ for all $k \in \mathbb{N}_{0}$. As in [21], the proof of Theorem 1.3 relies on two steps: the first is to prove a central limit theorem for the sequence $\left\{\mu_{n}^{n}\right\}$ (with suitable scaling), and the second is to show that under the central limit scaling, the sequence $\left\{\rho_{n}^{n}\right\}$ tends to 0 in probability.

We follow a similar approach for Algorithm II introduced in (1.8). This time we define the error and remainder sequences $\left\{\boldsymbol{e}_{n+1}\right\}$ and $\left\{\boldsymbol{r}_{n+1}\right\}$ by

$$
\boldsymbol{e}_{n+1} \doteq \frac{1}{a(n+1)} \sum_{i=1}^{a(n+1)} e_{n+1}^{i}, \quad \boldsymbol{r}_{n+1} \doteq \frac{1}{a(n+1)} \sum_{i=1}^{a(n+1)} r_{n+1}^{i}
$$

where the terms for each particle are given by

$$
\begin{aligned}
& e_{n+1}^{i} \doteq Q\left[\boldsymbol{\theta}_{n}\right]_{\boldsymbol{X}_{n+1}^{i},}-\left(K\left[\boldsymbol{\theta}_{n}\right] Q\left[\boldsymbol{\theta}_{n}\right]\right)_{\boldsymbol{X}_{n}^{i},}, \\
& r_{n+1}^{i} \doteq\left(K\left[\boldsymbol{\theta}_{n}\right] Q\left[\boldsymbol{\theta}_{n}\right]\right)_{\boldsymbol{X}_{n}^{i}, \cdot}-\left(K\left[\boldsymbol{\theta}_{n}\right] Q\left[\boldsymbol{\theta}_{k}\right]\right)_{\boldsymbol{X}_{n+1}^{i},},
\end{aligned}
$$

Then the sequence $\left\{\boldsymbol{\theta}_{n}\right\}$ defined in (1.8) can be rewritten as

$$
\boldsymbol{\theta}_{n+1}=\boldsymbol{\theta}_{n}+\gamma_{n+1} h\left(\boldsymbol{\theta}_{n}\right)+\gamma_{n+1} \boldsymbol{e}_{n+1}+\gamma_{n+1} \boldsymbol{r}_{n+1} .
$$

We also introduce the linearized evolution sequence $\left\{\boldsymbol{\mu}_{k}\right\}$ given by

$$
\boldsymbol{\mu}_{0} \doteq 0 \quad \text { and } \quad \boldsymbol{\mu}_{n+1} \doteq\left(I+\gamma_{n+1} \nabla h\left(\theta_{*}\right)\right) \boldsymbol{\mu}_{n}+\gamma_{n+1} \boldsymbol{e}_{n+1}
$$

and we define the discrepancy sequence $\left\{\boldsymbol{\rho}_{k}\right\}$ by

$$
\boldsymbol{\rho}_{0} \doteq \boldsymbol{\theta}_{0}-\theta_{*} \quad \text { and } \quad \boldsymbol{\rho}_{n+1} \doteq \boldsymbol{\theta}_{n+1}-\theta_{*}-\boldsymbol{\mu}_{n+1} \text {. }
$$

The proof once more proceeds by first establishing a central limit theorem for the linearized evolution and then showing that the discrepancy is asymptotically negligible.

\subsection{Notation}

The following notation will be used. Convergence in distribution of random variables $Z_{n}$ to $Z$ will be denoted as $Z_{n} \stackrel{\mathcal{L}}{\rightarrow} Z$. Constants in the proofs of various estimates will be denoted as $\kappa, \kappa_{1}, \kappa_{2}, \cdots$; their values may change from one proof to next. For a space $S, m \in \mathbb{N}$ and a bounded $h: S \rightarrow \mathbb{R}^{m},\|h\|_{\infty} \doteq \sup _{s \in S}\|h(s)\|$. For nonnegative sequences $\left\{a_{n}\right\},\left\{b_{n}\right\}$ we write $a_{n} \sim b_{n}$, if $a_{n} / b_{n} \rightarrow 1$ as $n \rightarrow \infty$. For a vector $v \in \mathbb{R}^{d}$, the $j$-th coordinate will be denoted as $v(j)$ or $v_{j}$. We denote by $\mathcal{C}^{0} \doteq C^{0}\left(\mathbb{R}_{+}, \mathcal{P}\left(\Delta^{o}\right)\right)$ the space of continuous $\mathcal{P}\left(\Delta^{o}\right)$ valued functions on $[0, \infty)$ endowed with the topology of uniform convergence on compact intervals. Recall that a sequence $\left\{x_{n}\right\}$ from $\mathbb{R}_{+}$to $\mathcal{P}\left(\Delta^{o}\right)$ converges to a limit $x_{*}$ in $\mathcal{C}^{0}$ if and only if for each $T>0$,

$$
\lim _{n \rightarrow \infty} \sup _{0 \leq s \leq T}\left\|x_{n}(t)-x_{*}(t)\right\|=0 .
$$

For $x \in \mathcal{C}^{0}$ we let $\|x\|_{T, *} \doteq \sup _{0 \leq s \leq T}\|x(s)\|$. Recall that the topology on $\mathcal{C}^{0}$ is induced by the metric

$$
d(x, y) \doteq \sum_{T=1}^{\infty} 2^{-T} \min \left\{1,\|x-y\|_{T, *}\right\}, \quad x, y \in \mathcal{C}^{0}
$$




\subsection{Organization}

The paper is organized as follows. In Section 2 we prove a.s. convergence of Algorithm I and provide some associated rate of convergence bounds (Thm. 1.2). In Section 3 we analyze the noise terms of Algorithm I. Combining results of Sections 2 and 3, in Section 4 we prove the central limit theorem for this algorithm stated in Theorem 1.3. In Section 5 we prove a.s. convergence for Algorithm II and in Section 6 we establish the corresponding CLT. Finally, in Section 7 we present some exploratory numerical experiments.

\section{Convergence of Algorithm I}

This section is dedicated to the proof of Theorem 1.2. In Section 2.1 we introduce a notion of pseudo-trajectory sequences for the flow induced by $h$ that is motivated by ideas of asymptotic pseudo trajectories introduced first in [8] (see also [4, 6]) and which is more well-suited for the array-type stochastic approximations studied here. In Section 2.2 we show that the sequence $\left\{\hat{\theta}^{n}\right\}$ of continuous time processes obtained from a suitable interpolation of our stochastic approximation array $\left\{\theta_{k}^{n}\right\}$ satisfies the pseudo-trajectory sequence property introduced in Section 2.1 and finally, in Section 2.3 we use this fact to complete the proof of Theorem 1.2.

\subsection{Pseudo-trajectory sequences}

Consider the sequence of algorithm update time instants $\left\{\tau_{k}\right\}$ associated with the SA, defined as

$$
\tau_{0}=0, \tau_{k} \doteq \sum_{j=1}^{k} \gamma_{j}, k \in \mathbb{N}
$$

For $r \in \mathbb{R}_{+}$, we let $\tau_{r} \doteq \tau_{\lfloor r\rfloor}$. For $\nu \in \mathcal{P}\left(\Delta^{o}\right)$, consider the ODE associated with the flow induced by $h$,

$$
\dot{\Phi}(t)=h(\Phi(t)), \quad \Phi(0)=\nu
$$

We denote the solution to (2.2) with initial condition $\Phi(0)=\nu$ by $\left\{\Phi_{t}(\nu)\right\}$.

We now introduce a notion of a pseudo-trajectory sequence that will be convenient for our purposes.

Definition 2.1. For $\lambda<0$ and $p \in(0,1]$, we say that a sequence $\left\{X_{n}\right\} \subset \mathcal{C}^{0}$ is a $\left(\lambda, \tau_{n}, p\right)$-pseudo-trajectory sequence (PTS) for $\Phi$ if for all $T>0$ and $\epsilon>0$, there is an $n_{0} \in \mathbb{N}$ such that for all $n \geq n_{0}$ and $0 \leq j \leq L_{n} \doteq$ $L_{n}(p, T) \doteq\left\lfloor\frac{1}{T}\left(\tau_{n}-\frac{\tau_{n} p}{2}\right)\right\rfloor+1$, and $t_{n, j} \doteq \frac{\tau_{n} p}{2}+j T$,

$$
\sup _{0 \leq u \leq 2 T}\left\|X_{n}\left(t_{n, j}+u\right)-\Phi_{u}\left(X_{n}\left(t_{n, j}\right)\right)\right\| \leq \exp \left\{(\lambda+\epsilon) t_{n, j}\right\}
$$

The following lemma provides an upper bound for the rate at which a $\left(\lambda, \tau_{n}, p\right)$-PTS converges to $\theta_{*}$. Recall that the largest eigenvalue of $\nabla h\left(\theta_{*}\right)$ is bounded above by $-L<0$.

Lemma 2.2. Suppose that for some $\lambda<0$ and $p \in(0,1),\left\{X_{n}\right\}$ is a $\left(\lambda, \tau_{n}, p\right)$-PTS for $\Phi$. Then there is some $\beta>0$ and $n_{0} \in \mathbb{N}$ such that for all $n \geq n_{0}$, if $n^{p} \leq m \leq n$, then

$$
\left\|X_{n}\left(\tau_{m}\right)-\theta_{*}\right\| \leq \exp \left(-\beta \tau_{m}\right)
$$

Proof. Fix $\alpha_{1} \in(0, L)$. Then we can find (cf. [6], Lem. 2.1) some $T \in(0, \infty)$ so that for all $\nu \in \mathcal{P}\left(\Delta^{o}\right)$,

$$
\sup _{T \leq u \leq 2 T}\left\|\Phi_{u}(\nu)-\theta_{*}\right\| \leq \exp \left(-\alpha_{1} T\right)\left\|\nu-\theta_{*}\right\|
$$


For $n^{p} \leq m \leq n$, let $0 \leq j(m) \leq L_{n}$ be such that $t_{n, j(m)+1} \leq \tau_{m} \leq t_{n, j(m)+1}+T$ and let $u(m) \doteq \tau_{m}-t_{n, j(m)}$, so that

$$
\tau_{m}=\left(\tau_{m}-t_{n, j(m)}\right)+t_{n, j(m)}=u(m)+t_{n, j(m)} .
$$

Note that $u(m) \in[T, 2 T]$. Now, fix $\epsilon \in(0,-\lambda)$, and let $\alpha_{2} \doteq-(\lambda+\epsilon)>0$. Define $\alpha \doteq \alpha_{1} \wedge \alpha_{2}$. Since $\left\{X_{n}\right\}$ is a $\left(\lambda, \tau_{n}, p\right)$-PTS for $\Phi$, we can find some $n_{0}$ such that for all $n \geq n_{0}$ and for each $n^{p} \leq m \leq n$,

$$
\begin{aligned}
\left\|X_{n}\left(\tau_{m}\right)-\theta_{*}\right\| & \leq\left\|X_{n}\left(u(m)+t_{n, j(m)}\right)-\Phi_{u(m)}\left(X_{n}\left(t_{n, j(m)}\right)\right)\right\|+\left\|\Phi_{u(m)}\left(X_{n}\left(t_{n, j(m)}\right)\right)-\theta_{*}\right\| \\
& \leq \exp \left(-\alpha t_{n, j(m)}\right)+\exp (-\alpha T)\left\|X_{n}\left(t_{n, j(m)}\right)-\theta_{*}\right\| \\
& \leq \exp \left(-\alpha \frac{\tau_{n^{p}}}{2}\right)+\exp (-\alpha T)\left\|X_{n}\left(t_{n, j(m)}\right)-\theta_{*}\right\| .
\end{aligned}
$$

Iterating this for an additional $j(m)$ times, we see that there are $\kappa_{i} \equiv \kappa_{i}(\alpha, T) \in(0, \infty)$ such that if $n \geq n_{0}$ and $m \geq n^{p}$, then

$$
\begin{aligned}
\left\|X_{n}\left(\tau_{m}\right)-\theta_{*}\right\| & \leq \exp \left(-\alpha \frac{\tau_{n^{p}}}{2}\right)\left(\sum_{k=0}^{j(m)} \exp (-\alpha k T)\right)+\kappa_{1} \exp (-\alpha(j(m)+1) T) \\
& \leq \kappa_{2}\left(\exp \left(-\alpha \frac{\tau_{n^{p}}}{2}\right)+\exp (-\alpha(j(m)+1) T)\right) \\
& =\kappa_{2}\left(\exp \left(-\alpha \frac{\tau_{n^{p}}}{2}\right)+\exp (-\alpha(j(m)+2) T) \exp (\alpha T)\right) \\
& \leq \kappa_{3}\left(\exp \left(-\alpha \frac{\tau_{n^{p}}}{2}\right)+\exp (-\alpha(j(m)+2) T)\right)
\end{aligned}
$$

Note that, by our choice of $j(m)$,

$$
T(j(m)+2) \geq \tau_{m}-\frac{\tau_{n^{p}}}{2} \geq \frac{\tau_{n^{p}}}{2} .
$$

Also note that for $k \in \mathbb{N}$

$$
\gamma_{*}\left(\log \left(k+N_{*}\right)-\log \left(N_{*}\right)\right) \leq \tau_{k} \leq 1+\gamma_{*} \log \left(k+N_{*}-1\right)
$$

from which it follows that, there is a $\kappa_{4} \in(0, \infty)$ and $n_{1} \geq n_{0}$ such that and all $n \geq n_{1}, \frac{\tau_{n} p}{2} \geq \kappa_{4} \tau_{n}$. Combining the above two observations with (2.3), we have for all $n \geq n_{1}$ and $n^{p} \leq m \leq n$

$$
\left\|X_{n}\left(\tau_{m}\right)-\theta_{*}\right\| \leq 2 \kappa_{3} \exp \left(-\alpha \frac{\tau_{n^{p}}}{2}\right) \leq 2 \kappa_{3} \exp \left(-\alpha \kappa_{4} \tau_{m}\right)
$$

The result follows.

\subsection{The algorithm as a pseudo-trajectory sequence}

In this section we show that a suitable continuous time interpolation of the array $\left\{\theta_{k}^{n}\right\}$ is a PTS for $\Phi$ in the sense of Definition 2.1. For $n \in \mathbb{N}$, let $\hat{\theta}^{n}$ be the continuous-time process defined as

$$
\hat{\theta}^{n}\left(\tau_{k}+t\right) \doteq \theta_{k}^{n}+t \frac{\theta_{k+1}^{n}-\theta_{k}^{n}}{\tau_{k+1}-\tau_{k}}, t \in\left[0, \gamma_{k+1}\right) \text { and } k \in \mathbb{N}_{0}
$$


We write $\bar{\theta}^{n}(\cdot)$ to denote the analogous continuous-time process obtained by piecewise constant interpolations of $\left\{\theta_{k}^{n}\right\}$. We will prove in this section that, with $\lambda=-\left(2 \gamma_{*}\right)^{-1}$ and arbitrary $p \in(0,1),\left\{\hat{\theta}^{n}\right\}$ is a $\left(\lambda, \tau_{n}, p\right)$-PTS for $\Phi$. Towards that end, let

$$
\bar{\epsilon}^{n}\left(\tau_{k}+t\right) \doteq \epsilon_{k+1}^{n}, \quad t \in\left[0, \gamma_{k+1}\right), k \in \mathbb{N}_{0}
$$

and define

$$
\Delta\left(n, t_{n, j}, T\right) \doteq \sup _{0 \leq u \leq 2 T}\left\|\int_{t_{n, j}}^{t_{n, j}+u} \bar{\epsilon}^{n}(s) \mathrm{d} s\right\|, \quad 0 \leq j \leq L_{n}
$$

where $L_{n}=L_{n}(p, T)$ is as in Definition 2.1. In Lemma 2.3 we provide an estimate relating $\hat{\theta}^{n}$ with $\Delta(\cdot, \cdot, \cdot)$ that is used to prove asymptotic properties of $\left\{\hat{\theta}^{n}\right\}$. The proof is a consequence of the Lipschitz property of $h$ and Grönwall's lemma. Define $m: \mathbb{R}_{+} \rightarrow \mathbb{N}_{0}$ by

$$
m(t) \doteq \sup \left\{k \geq 0: t \geq \tau_{k}\right\}, t \geq 0 .
$$

Lemma 2.3. For each $T \in(0, \infty)$ there is a $C \doteq C(T) \in(0, \infty)$ such that for all $n \in \mathbb{N}$ and $k \leq L_{n}$,

$$
\sup _{0 \leq u \leq 2 T}\left\|\hat{\theta}^{n}\left(t_{n, k}+u\right)-\Phi_{u}\left(\hat{\theta}^{n}\left(t_{n, k}\right)\right)\right\| \leq C(T)\left[\Delta\left(n, t_{n, k}, T\right)+\gamma_{m\left(t_{n, k}\right)}\right]
$$

Proof. Fix $T \in(0, \infty)$. Note that, for $n \in \mathbb{N}$ and $t \geq 0$,

$$
\hat{\theta}^{n}(t)=\hat{\theta}^{n}(0)+\int_{0}^{t}\left[h\left(\bar{\theta}^{n}(s)\right)+\bar{\epsilon}^{n}(s)\right] \mathrm{d} s .
$$

Define

$$
A_{n, k}(s) \doteq \int_{t_{n, k}}^{t_{n, k}+s}\left[h\left(\bar{\theta}^{n}(u)\right)-h\left(\hat{\theta}^{n}(u)\right)\right] \mathrm{d} u, \quad B_{n, k}(s) \doteq \int_{t_{n, k}}^{t_{n, k}+s} \bar{\epsilon}^{n}(u) \mathrm{d} u .
$$

Then, for $0 \leq s \leq 2 T$,

$$
\hat{\theta}^{n}\left(t_{n, k}+s\right)=\hat{\theta}^{n}\left(t_{n, k}\right)+\int_{0}^{s} h\left(\hat{\theta}^{n}\left(t_{n, k}+u\right)\right) \mathrm{d} u+A_{n, k}(s)+B_{n, k}(s) .
$$

Also,

$$
\Phi_{s}\left(\hat{\theta}^{n}\left(t_{n, k}\right)\right)=\hat{\theta}^{n}\left(t_{n, k}\right)+\int_{0}^{s} h\left(\Phi_{u}\left(\hat{\theta}^{n}\left(t_{n, k}\right)\right)\right) \mathrm{d} u
$$

Letting $K$ denote the Lipschitz constant of $h$ we see that

$$
\begin{aligned}
\left\|\hat{\theta}^{n}\left(t_{n, k}+s\right)-\Phi_{s}\left(\hat{\theta}^{n}\left(t_{n, k}\right)\right)\right\| & =\left\|\int_{0}^{s}\left[h\left(\hat{\theta}^{n}\left(t_{n, k}+u\right)\right)-h\left(\Phi_{u}\left(\hat{\theta}^{n}\left(t_{n, k}\right)\right)\right)\right] \mathrm{d} u+A_{n, k}(s)+B_{n, k}(s)\right\| \\
& \leq K \int_{0}^{s}\left\|\hat{\theta}^{n}\left(t_{n, k}+u\right)-\Phi_{u}\left(\hat{\theta}^{n}\left(t_{n, k}\right)\right)\right\| \mathrm{d} u+\left\|A_{n, k}(s)\right\|+\left\|B_{n, k}(s)\right\|,
\end{aligned}
$$


for all $s \geq 0$. Next for each $u \in\left[t_{n, k}, t_{n, k}+2 T\right]$,

$$
\hat{\theta}^{n}(u)-\bar{\theta}^{n}(u)=\hat{\theta}^{n}(u)-\hat{\theta}^{n}\left(\tau_{m(u)}\right)=\int_{\tau_{m(u)}}^{u}\left[h\left(\bar{\theta}^{n}(s)\right)+\bar{\epsilon}^{n}(s)\right] \mathrm{d} s .
$$

Note that, with $\kappa_{1} \doteq\|h\|_{\infty}+2$, for $u \in\left[t_{n, k}, t_{n, k}+2 T\right]$,

$$
\left\|\int_{\tau_{m(u)}}^{u}\left(h\left(\bar{\theta}^{n}(s)\right)+\bar{\epsilon}^{n}(s)\right) \mathrm{d} s\right\| \leq \kappa_{1}\left(u-\tau_{m(u)}\right) \leq \kappa_{1} \gamma_{m(u)} \leq \kappa_{1} \gamma_{m\left(t_{n, k}\right)},
$$

Combining the above estimate with (2.8), it follows that for $0 \leq s \leq 2 T$,

$$
\left\|A_{n, k}(s)\right\| \leq K \int_{t_{n, k}}^{t_{n, k}+s}\left\|\bar{\theta}^{n}(u)-\hat{\theta}^{n}(u)\right\| \mathrm{d} u \leq 2 K T \kappa_{1} \gamma_{m\left(t_{n, k}\right)} .
$$

The result now follows on using the estimate (2.9) in (2.7), recalling the definition of $B_{n, k}$ and $\Delta\left(n, t_{n, k}, T\right)$, and applying Grönwall's lemma.

Lemma 2.9 provides the key estimate in the proof that $\left\{\hat{\theta}^{n}\right\}$ is a PTS for $\Phi$. The main ingredients in its proof are Lemmas 2.4, 2.5, 2.6, and 2.7 given below. Consider the following decomposition of the algorithm's noise given in terms of $\delta_{k+1}^{\ell, i, n}$ defined as, for $1 \leq i \leq a(n)$,

$$
\delta_{k+1}^{\ell, i, n}(x) \doteq\left\{\begin{array}{lr}
\gamma_{k+1} Q\left[\theta_{k}^{n}\right]_{X_{k+1}^{i}, x}-\gamma_{k+1}\left(K\left[\theta_{k}^{n}\right] Q\left[\theta_{k}^{n}\right]\right)_{X_{k}^{i}, x} & \ell=1 \\
\gamma_{k+1}\left(K\left[\theta_{k}^{n}\right] Q\left[\theta_{k}^{n}\right]\right)_{X_{k}^{i}, x}-\gamma_{k}\left(K\left[\theta_{k}^{n}\right] Q\left[\theta_{k}^{n}\right]\right)_{X_{k}^{i}, x} & \ell=2 \\
\gamma_{k}\left(K\left[\theta_{k}^{n}\right] Q\left[\theta_{k}^{n}\right]\right)_{X_{k}^{i}, x}-\gamma_{k+1}\left(K\left[\theta_{k+1}^{n}\right] Q\left[\theta_{k+1}^{n}\right]\right)_{X_{k+1}^{i}, x} & \ell=3 \\
\gamma_{k+1}\left(K\left[\theta_{k+1}^{n}\right] Q\left[\theta_{k+1}^{n}\right]\right)_{X_{k+1}^{i}, x}-\gamma_{k+1}\left(K\left[\theta_{k}^{n}\right] Q\left[\theta_{k}^{n}\right]\right)_{X_{k+1}^{i}, x} & \ell=4
\end{array}\right.
$$

For each $1 \leq \ell \leq 4$, let

$$
\delta_{k+1}^{\ell, n} \doteq \frac{1}{a(n)} \sum_{i=1}^{a(n)} \delta_{k+1}^{\ell, i, n} \quad \text { and observe that } \quad \gamma_{k+1} \epsilon_{k+1}^{n}=\sum_{\ell=1}^{4} \delta_{k+1}^{\ell, n}
$$

since

$$
\gamma_{k+1} \epsilon_{k+1}^{i, n}=\gamma_{k+1}\left(Q\left[\theta_{k}^{n}\right]_{X_{k+1}^{i}, x}-\left(K\left[\theta_{k}^{n}\right] Q\left[\theta_{k}^{n}\right]\right)_{X_{k+1}^{i}, x}\right)=\sum_{\ell=1}^{4} \delta_{k+1}^{\ell, i, n}
$$

The following lemma estimates the error term corresponding to $\ell=1$. Henceforth in this section we assume that $p \in(0,1)$ and $T \in(0, \infty)$ are fixed, and $\lambda \doteq-\left(2 \gamma_{*}\right)^{-1}$. Recall the quantities $L_{n}$ and $t_{n, j}$ from Definition 2.1.

Lemma 2.4. Let $q \geq 2$. There is a $n_{0} \in \mathbb{N}$ and $C(q, T) \in(0, \infty)$ such that for all $n \geq n_{0}$ and all $0 \leq k \leq L_{n}$,

$$
\mathbf{E}\left(\sup _{0 \leq u \leq 2 T}\left\|\sum_{j=m\left(t_{n, k}\right)}^{m\left(t_{n, k}+u\right)} \delta_{j+1}^{1, n}\right\|^{q}\right) \leq C(q, T) \exp \left(q \lambda t_{n, k}\right)
$$


Proof. Note that for each $n \in \mathbb{N},\left\{\delta_{j}^{1, n}\right\}_{j=1}^{\infty}$ is adapted to $\left\{\mathcal{F}_{j}^{n}\right\}_{j=1}^{\infty}$. Additionally, from (1.3), for all $n \in \mathbb{N}, j \in \mathbb{N}_{0}$,

$$
\mathbf{E}\left[\delta_{j+1}^{1, n} \mid \mathcal{F}_{j}\right]=\gamma_{j+1} \frac{1}{a(n)} \sum_{i=1}^{a(n)} \mathbf{E}\left[\left(Q\left[\theta_{j}^{n}\right]_{X_{j+1}^{i, n},}-K\left[\theta_{j}^{n}\right] Q\left[\theta_{j}^{n}\right]_{X_{j}^{i, n}, .}\right) \mid \mathcal{F}_{j}^{n}\right]=0
$$

Also, for all $n \in \mathbb{N}$ and $j \in \mathbb{N}_{0}$,

$$
\left\|\delta_{j+1}^{1, n}\right\| \leq \frac{1}{a(n)} \sum_{i=1}^{a(n)}\left\|\delta_{j+1}^{1, i, n}\right\| \leq \gamma_{j+1} \frac{1}{a(n)} \sum_{i=1}^{a(n)}\left\|Q\left[\theta_{j}^{n}\right]_{X_{j+1}^{i, n}, \cdot}-K\left[\theta_{j}^{n}\right] Q\left[\theta_{j}^{n}\right]_{X_{j}^{i, n}, \cdot}\right\| \leq \kappa_{1} \gamma_{j+1}
$$

for some $\kappa_{1} \in(0, \infty)$. Thus, for each $n \in \mathbb{N},\left\{\delta_{j}^{1, n}\right\}_{j=1}^{\infty}$ is a martingale difference sequence, and so from Burkholder's inequality we can find a $\kappa_{2}(q) \in(0, \infty)$ such that for all $n \in \mathbb{N}$ and $0 \leq k \leq L_{n}$,

$$
\begin{aligned}
f_{n, k}(q) & \doteq \mathbf{E}\left(\sup _{0 \leq u \leq 2 T}\left\|\sum_{i=m\left(t_{n, k}\right)}^{m\left(t_{n, k}+u\right)} \delta_{i+1}^{1, n}\right\|^{q}\right) \leq \mathbf{E}\left(\sup _{m\left(t_{n, k}\right) \leq j \leq m\left(t_{n, k}+2 T\right)}\left\|\sum_{i=m\left(t_{n, k}\right)}^{j} \delta_{i+1}^{1, n}\right\|^{q}\right) \\
& \leq \kappa_{2}(q) \mathbf{E}\left(\left[\sum_{i=m\left(t_{n, k}\right)}^{m\left(t_{n, k}+2 T\right)}\left\|\delta_{i+1}^{1, n}\right\|^{2}\right]^{q / 2}\right) \leq \kappa_{2}(q) \kappa_{1}^{q}\left[\sum_{i=m\left(t_{n, k}\right)}^{m\left(t_{n, k}+2 T\right)} \gamma_{i+1}^{2}\right]^{q / 2} \\
& \leq \kappa_{2}(q) \kappa_{1}^{q}\left[m\left(t_{n, k}+2 T\right)-m\left(t_{n, k}\right)+1\right]^{q / 2} \gamma_{m\left(t_{n, k}\right)}^{q} .
\end{aligned}
$$

Next note that, for some $\kappa_{3}(T) \in(0, \infty)$ and $n_{0} \in \mathbb{N}$,

$$
\left[m\left(t_{n, k}+2 T\right)-m\left(t_{n, k}\right)+1\right] \leq \kappa_{3}(T) \exp \left(\frac{t_{n, k}}{\gamma_{*}}\right)
$$

and for all $n \geq n_{0}$

$$
\gamma_{m\left(t_{n, k}\right)} \leq \frac{\gamma_{*} e}{N_{*}} \exp \left(-\frac{t_{n, k}}{\gamma_{*}}\right)
$$

Thus, there is some $\kappa_{4}(q, T) \in(0, \infty)$ such that for all $n \geq n_{0}$,

$$
f_{n, k}(q) \leq \kappa_{4}(q, T) \exp \left(-\frac{q}{2 \gamma_{*}} t_{n, k}\right)=\kappa_{4}(q, T) \exp \left(\lambda q t_{n, k}\right) .
$$

The result follows.

The next three lemmas, namely Lemmas 2.5, 2.6, and 2.7 will be used to bound the remaining error terms.

Lemma 2.5. There is a $C \in(0, \infty)$ such that for all $T>0, n \in \mathbb{N}$ and $k \leq L_{n}$

$$
\sup _{0 \leq u \leq 2 T}\left\|\sum_{j=m\left(t_{n, k}\right)}^{m\left(t_{n, k}+u\right)} \delta_{j+1}^{2, n}\right\| \leq C \gamma_{m\left(t_{n, k}\right)} .
$$


Proof. Fix $n \in \mathbb{N}$ and let

$$
\kappa \doteq \sup _{\theta \in \mathcal{P}\left(\Delta^{o}\right), x \in \Delta^{o}}\left\|(K[\theta] Q[\theta])_{x, \cdot}\right\|<\infty
$$

Then for each $1 \leq i \leq a(n),\left\|\delta_{j+1}^{2, i, n}\right\| \leq \kappa\left(\gamma_{j+1}-\gamma_{j}\right)$ for all $j \in \mathbb{N}_{0}$, and so for each $k \leq L_{n}$,

$$
\sup _{0 \leq u \leq 2 T}\left\|\sum_{j=m\left(t_{n, k}\right)}^{m\left(t_{n, k}+u\right)} \delta_{j+1}^{2, n}\right\| \leq \sup _{0 \leq u \leq 2 T} \sum_{j=m\left(t_{n, k}\right)}^{m\left(t_{n, k}+u\right)} \frac{1}{a(n)} \sum_{i=1}^{a(n)}\left\|\delta_{j+1}^{2, i, n}\right\| \leq \kappa \gamma_{m\left(t_{n, k}\right)} .
$$

The result follows.

Lemma 2.6. There is a $C \in(0, \infty)$ such that for all $T>0, n \in \mathbb{N}$ and $0 \leq k \leq L_{n}$.

$$
\sup _{0 \leq u \leq 2 T}\left\|\sum_{j=m\left(t_{n, k}\right)}^{m\left(t_{n, k}+u\right)} \delta_{j+1}^{3, n}\right\| \leq C \gamma_{m\left(t_{n, k}\right)}
$$

Proof. Let $b_{j}^{i, n}(\cdot) \doteq \gamma_{j} K\left[\theta_{j}^{n}\right] Q\left[\theta_{j}^{n}\right]_{X_{j}^{i}, \text {, }}$ so that $\delta_{j+1}^{3, i, n}=b_{j}^{i, n}-b_{j+1}^{i, n}$. Then, with $\kappa$ as in $(2.16)$, we have that $\left\|b_{j}^{i, n}\right\| \leq \kappa \gamma_{j}$. Thus

$$
\begin{aligned}
\left\|\sum_{j=m\left(t_{n, k}\right)}^{m\left(t_{n, k}+u\right)} \delta_{j+1}^{3, n}\right\| & =\left\|\sum_{j=m\left(t_{n, k}\right)}^{m\left(t_{n, k}+u\right)} \frac{1}{a(n)} \sum_{i=1}^{a(n)}\left(b_{j}^{i, n}-b_{j+1}^{i, n}\right)\right\| \\
& \leq \frac{1}{a(n)} \sum_{i=1}^{a(n)}\left\|b_{m\left(t_{n, k}\right)}^{i, n}-b_{m\left(t_{n, k}+u\right)+1}^{i, n}\right\| \leq 2 \kappa \gamma_{m\left(t_{n, k}\right)} .
\end{aligned}
$$

The result follows.

Lemma 2.7. Fix $T \in(0, \infty)$. There is a $C \in(0, \infty)$ such that for all $n \in \mathbb{N}$ and $0 \leq k \leq L_{n}$

$$
\sup _{0 \leq u \leq 2 T}\left\|\sum_{j=m\left(t_{n, k}\right)}^{m\left(t_{n, k}+u\right)} \delta_{j+1}^{4, n}\right\| \leq C \gamma_{m\left(t_{n, k}\right)}
$$

Proof. Using the boundedness and Lipschitz property of $K(\cdot)$ and $Q(\cdot)$, we see that for some $\kappa_{1} \in(0, \infty)$, $\left\|\delta_{j+1}^{4, i, n}\right\| \leq \kappa_{1} \gamma_{j+1}\left\|\theta_{j+1}^{n}-\theta_{j}^{n}\right\|$. Also, from (1.6),

$$
\left\|\theta_{j+1}^{n}-\theta_{j}^{n}\right\|=\gamma_{j+1}\left\|h\left(\theta_{j}^{n}\right)+\epsilon_{j+1}^{n}\right\| \leq 3 \gamma_{j+1} .
$$

Thus, for $0 \leq u \leq 2 T$,

$$
\begin{aligned}
\left\|\sum_{j=m\left(t_{n, k}\right)}^{m\left(t_{n, k}+u\right)} \delta_{j+1}^{4, n}\right\|=\left\|\sum_{j=m\left(t_{n, k}\right)}^{m\left(t_{n, k}+u\right)} \frac{1}{a(n)} \sum_{i=1}^{a(n)} \delta_{j+1}^{4, i, n}\right\| & \leq 3 \kappa_{1} \sum_{j=m\left(t_{n, k}\right)}^{m\left(t_{n, k}+u\right)} \gamma_{j+1}^{2} \\
& \leq 3 \kappa_{1} \gamma_{m\left(t_{n, k}\right)+1}^{2}\left[m\left(t_{n, k}+2 T\right)-m\left(t_{n, k}\right)+1\right] .
\end{aligned}
$$


The result now follows on noting that from (2.14) and (2.15), for some $\kappa_{2} \in(0, \infty)$

$$
\gamma_{m\left(t_{n, k}\right)+1}^{2}\left[m\left(t_{n, k}+2 T\right)-m\left(t_{n, k}\right)+1\right] \leq \kappa_{2} \gamma_{m\left(t_{n, k}\right)} \exp \left(\frac{t_{n, k}}{\gamma_{*}}\right) \exp \left(-\frac{2 t_{n, k}}{\gamma_{*}}\right) \leq \kappa_{2} \gamma_{m\left(t_{n, k}\right)} .
$$

The following corollary is used in the proof of Lemma 2.9. Recall the definition of $r_{k+1}^{n}$ from (1.18). For a collection of events $\left\{A_{n, k}: 0 \leq k \leq L_{n}, n \in \mathbb{N}\right\}$ we denote

$$
\left\{A_{n, k} \text { i.o. }\right\} \doteq\left\{\omega: \omega \in A_{n, k} \text { for infinitely many }(n, k) \text {, s.t. } 0 \leq k \leq L_{n}, n \in \mathbb{N}\right\} \text {. }
$$

Corollary 2.8. Fix $T \in(0, \infty)$. Then, for each $C \in(0, \infty)$

$$
\mathbf{P}\left(\sup _{0 \leq u \leq 2 T}\left\|\sum_{j=m\left(t_{n, k}\right)}^{m\left(t_{n, k}+u\right)} \gamma_{j+1} r_{j+1}^{n}\right\|+C \gamma_{m\left(t_{n, k}\right)}>\frac{1}{2} \exp \left(\lambda t_{n, k}\right) \text { i.o. }\right)=0 .
$$

Proof. Fix $T, C \in(0, \infty)$. From Lemmas 2.5, 2.6, and 2.7, and $(2.15)$, for some $\kappa_{1} \in(0, \infty), n_{0} \in \mathbb{N}$, and all $n \geq n_{0}$,

$$
\begin{aligned}
\sup _{0 \leq u \leq 2 T}\left\|\sum_{j=m\left(t_{n, k}\right)}^{m\left(t_{n, k}+u\right)} \gamma_{j+1} r_{j+1}^{n}\right\|+C \gamma_{m\left(t_{n, k}\right)} & \leq \sum_{\ell=2}^{4} \sup _{0 \leq u \leq 2 T}\left\|\sum_{j=m\left(t_{n, k}\right)}^{m\left(t_{n, k}+u\right)} \delta_{j+1}^{\ell, n}\right\|+C \gamma_{m\left(t_{n, k}\right)} \\
& \leq \kappa_{1} \gamma_{m\left(t_{n, k}\right)} \leq \frac{\kappa_{1} \gamma_{*} e}{N_{*}} \exp \left(-\frac{t_{n, k}}{\gamma_{*}}\right)
\end{aligned}
$$

The last expression can be bounded by $\frac{1}{2} \exp \left(\lambda t_{n, k}\right)$ for $n$ sufficiently large. The result follows.

We now present the key estimate that will be used to prove Theorem 1.2.

Lemma 2.9. For each $T>0$,

$$
\limsup _{n \rightarrow \infty} \sup _{k \leq L_{n}} \frac{1}{t_{n, k}} \log \Delta\left(n, t_{n, k}, T\right) \leq \lambda \quad \text { a.s. }
$$

Proof. Fix $T \in(0, \infty)$ and $\epsilon \in(0,-\lambda)$. Write $\sigma=\lambda+\epsilon$. From the boundedness of $\epsilon_{k+1}^{n}$, we can find $\kappa_{1} \in(0, \infty)$ such that

$$
\begin{aligned}
& \Delta\left(n, t_{n, k}, T\right)=\sup _{0 \leq u \leq 2 T}\left\|\int_{t_{n, k}}^{t_{n, k}+u} \bar{\epsilon}^{n}(s) \mathrm{d} s\right\|
\end{aligned}
$$

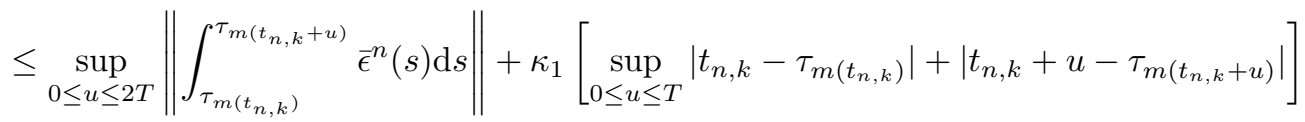

$$
\begin{aligned}
& \leq \sup _{0 \leq u \leq 2 T}\left\|\sum_{j=m\left(t_{n, k}\right)}^{m\left(t_{n, k}+u\right)} \gamma_{j+1} \epsilon_{j+1}^{n}\right\|+2 \kappa_{1} \gamma_{m\left(t_{n, k}\right)}
\end{aligned}
$$


From (2.12) it follows that

$$
\Delta\left(n, t_{n, k}, T\right) \leq \sup _{0 \leq u \leq 2 T}\left\|\sum_{j=m\left(t_{n, k}\right)}^{m\left(t_{n, k}+u\right)} \delta_{j+1}^{1, n}\right\|+\sup _{0 \leq u \leq 2 T}\left\|\sum_{j=m\left(t_{n, k}\right)}^{m\left(t_{n, k}+u\right)} \gamma_{j+1} r_{j+1}^{n}\right\|+2 \kappa_{1} \gamma_{m\left(t_{n, k}\right)} .
$$

Thus

$$
\begin{aligned}
\mathbf{P}\left(\Delta\left(n, t_{n, k}, T\right) \geq\right. & \left.\exp \left(t_{n, k} \sigma\right) \quad \text { i.o. }\right) \leq \mathbf{P}\left(\sup _{0 \leq u \leq 2 T}\left\|\sum_{j=m\left(t_{n, k}\right)}^{m\left(t_{n, k}+u\right)} \delta_{j+1}^{1, n}\right\|>\frac{1}{2} \exp \left(t_{n, k} \sigma\right) \quad \text { i.o. }\right) \\
& +\mathbf{P}\left(\sup _{0 \leq u \leq 2 T}\left\|\sum_{j=m\left(t_{n, k}\right)}^{m\left(t_{n, k}+u\right)} \gamma_{j+1} r_{j+1}^{n}\right\|+2 \kappa_{1} \gamma_{m\left(t_{n, k}\right)}>\frac{1}{2} \exp \left(t_{n, k} \sigma\right) \quad \text { i.o. }\right) .
\end{aligned}
$$

From Corollary 2.8, since $\sigma>\lambda$, the second term in (2.17) equals 0. Since $\epsilon \in(0,-\lambda)$ is arbitrary, in order to complete the proof of the lemma it now suffices to show that

$$
\mathbf{P}\left(\sup _{0 \leq u \leq 2 T}\left\|\sum_{j=m\left(t_{n, k}\right)}^{m\left(t_{n, k}+u\right)} \delta_{j+1}^{1, n}\right\|>\frac{1}{2} \exp \left(t_{n, k} \sigma\right) \text { i.o. }\right)=0 .
$$

Note that we can find some $\alpha_{0}>0$ and $n_{0} \in \mathbb{N}$ such that for all $n \geq n_{0}$ and all $k \leq L_{n}$,

$$
t_{n, k} \geq \frac{\tau_{n^{p}}}{2} \geq \alpha_{0} \log (n)
$$

Fix $q>\frac{1}{\epsilon \alpha_{0}} \vee 2$. Applying Lemma 2.4 and Markov's inequality, we can find $\kappa_{2}, \kappa_{3} \in(0, \infty)$ such that

$$
\begin{gathered}
\sum_{n=1}^{\infty} \sum_{k=1}^{L_{n}} \mathbf{P}\left(\sup _{0 \leq u \leq 2 T}\left\|\sum_{j=m\left(t_{n, k}\right)}^{m\left(t_{n, k}+u\right)} \delta_{j+1}^{1, n}\right\|>\frac{1}{2} \exp \left(t_{n, k} \sigma\right)\right) \\
\leq 2^{q} \kappa_{2} C(q, T) \sum_{n=1}^{\infty} \sum_{k=1}^{L_{n}} \exp \left(-q t_{n, k} \sigma\right) \exp \left(q t_{n, k} \lambda\right)=2^{q} \kappa_{2} C(q, T) \sum_{n=1}^{\infty} \sum_{k=1}^{L_{n}} \exp \left(-q t_{n, k} \epsilon\right) \\
\leq 2^{q} \kappa_{3} C(q, T) \sum_{n=1}^{\infty} \log (n) \exp \left(-q \epsilon \alpha_{0} \log (n)\right) \leq 2^{q} \kappa_{3} C(q, T) \sum_{n=1}^{\infty} \log (n) \frac{1}{n^{q \epsilon \alpha_{0}}}<\infty,
\end{gathered}
$$

where $C(q, T)$ is as in Lemma 2.4. The equation in (2.18) now follows from the Borel-Cantelli lemma and the result follows.

\subsection{Proof of Theorem 1.2}

We now complete the proof of Theorem 1.2. Fix $p \in(0,1)$. From Lemma 2.3, for every $T<\infty$, there is a $C(T) \in(0, \infty)$ such that for all $n \in \mathbb{N}$ and all $0 \leq k \leq L_{n}$,

$$
\sup _{0 \leq u \leq 2 T}\left\|\hat{\theta}^{n}\left(t_{n, k}+u\right)-\Phi_{u}\left(\hat{\theta}^{n}\left(t_{n, k}\right)\right)\right\| \leq C(T)\left[\Delta\left(n, t_{n, k}, T\right)+\gamma_{m\left(t_{n, k}\right)}\right) .
$$


Additionally, Lemma 2.9 ensures that for a.e. $\omega$, for every $\epsilon \in(0,-\lambda), T<\infty$, there is some $n_{1} \equiv n_{1}(\omega, \epsilon, T) \in \mathbb{N}$ such that for all $n \geq n_{1}$ and all $0 \leq k \leq L_{n}$,

$$
\Delta\left(n, t_{n, k}, T\right) \leq \exp \left(t_{n, k}(\lambda+\epsilon / 2)\right) .
$$

Combining this with (2.15) and (2.20), we have that for some $n_{2} \geq n_{1}$ and all $n \geq n_{2}, 0 \leq k \leq L_{n}$, we have

$$
\sup _{0 \leq u \leq 2 T}\left\|\hat{\theta}^{n}\left(t_{n, k}+u\right)-\Phi_{u}\left(\hat{\theta}^{n}\left(t_{n, k}\right)\right)\right\| \leq \exp \left((\lambda+\epsilon) t_{n, k}\right) .
$$

We have thus shown that $\left\{\hat{\theta}^{n}\right\}$ is a.s. a $\left(\lambda, \tau_{n}, p\right)$-pseudo-trajectory, so Lemma 2.2 ensures that there is some $\beta>0$ and $n_{0}=n_{0}(\omega) \in \mathbb{N}$ such that for all $n \geq n_{0}$ and $n^{p} \leq k \leq n$,

$$
\left\|\hat{\theta}^{n}\left(\tau_{k}\right)-\theta_{*}\right\| \leq \exp \left(-\beta \tau_{k}\right) .
$$

The result follows.

\section{Analysis of the noise terms in Algorithm I}

The goal of this section is to provide estimates on the error terms defined in (1.18) that will be useful for the study of the CLT. In Section 3.1 we characterize the covariance structure of the error terms $\left\{e_{k+1}^{n}\right\}$. In Section 3.2 we provide some bounds on the moments of $\left\{e_{k+1}^{n}\right\}$. Finally, in Section 3.3 we estimate the remainder terms $\left\{r_{k+1}^{n}\right\}$.

\subsection{Covariance structure of the error terms}

We first study the covariance structure of the error terms $\left\{e_{k+1}^{n}\right\}$. Consider the collection of $d \times d$ matrices $\left\{F_{\theta}(z): \theta \in \mathcal{P}\left(\Delta^{o}\right), z \in \Delta^{o}\right\}$ defined by, for $x, y \in \Delta^{o},(\theta, z) \in \mathcal{P}\left(\Delta^{o}\right) \times \Delta^{o}$,

$$
F_{\theta}(z)_{x, y} \doteq \sum_{u \in \Delta^{o}}\left(K[\theta]_{z, u} Q[\theta]_{u, x} Q[\theta]_{u, y}\right)-(K[\theta] Q[\theta])_{z, y}(K[\theta] Q[\theta])_{z, x},
$$

and let $U_{*}$ be the $d \times d$ matrix defined as

$$
U_{*} \doteq \sum_{w \in \Delta^{o}} F_{\theta_{*}}(w)\left(\theta_{*}\right)_{w}
$$

It is easily verified that $U_{*}$ is a nonnegative definite matrix. The following result gives an expression for the conditional covariance matrix of $e_{k+1}^{n}$.

Proposition 3.1. For each $n \in \mathbb{N}, 0 \leq k \leq n-1$, and $x, y \in \Delta^{o}$,

$$
\mathbf{E}\left[e_{k+1}^{n}(x) e_{k+1}^{n}(y) \mid \mathcal{F}_{k}^{n}\right]=\frac{1}{a(n)}\left(\left(U_{*}\right)_{x, y}+\left(D_{k}^{(1), n}\right)_{x, y}+\left(D_{k}^{(2), n}\right)_{x, y}\right),
$$

where the following hold:

(i) There is a $C_{1} \in(0, \infty)$ such that for all $n \in \mathbb{N}$ and $0 \leq k \leq n-1,\left\|D_{k}^{(1), n}\right\| \leq C_{1}\left\|\theta_{k}^{n}-\theta_{*}\right\|$.

(ii) There are some $C_{2}, \beta \in(0, \infty)$ such that for all $n \in \mathbb{N}$ and $1 \leq k \leq n$,

$$
\gamma_{k} \mathbf{E}\left\|\sum_{m=1}^{k} D_{m-1}^{(2), n}\right\| \leq C_{2} k^{-\beta}
$$


Proof. Fix $n \in \mathbb{N}$ and $0 \leq k \leq n-1$. Then, from (1.3), for each $x, y \in \Delta^{o}$ and $1 \leq i \leq a(n)$,

$$
\mathbf{E}\left[Q\left[\theta_{k}^{n}\right]_{X_{k+1}^{i, n}, x} \mid \mathcal{F}_{k}^{n}\right]=\left(K\left[\theta_{k}^{n}\right] Q\left[\theta_{k}^{n}\right]\right)_{X_{k}^{i, n}, x},
$$

and

$$
\mathbf{E}\left[Q\left[\theta_{k}^{n}\right]_{X_{k+1}^{i, n}, x} Q\left[\theta_{k}^{n}\right]_{X_{k+1}^{i, n}, y} \mid \mathcal{F}_{k}^{n}\right]=\sum_{w \in \Delta^{o}} K\left[\theta_{k}^{n}\right]_{X_{k}^{i, n}, w} Q\left[\theta_{k}^{n}\right]_{w, x} Q\left[\theta_{k}^{n}\right]_{w, y}
$$

Similarly, for each $x, y \in \Delta^{o}$, if $1 \leq i \neq j \leq a(n)$, then

$$
\mathbf{E}\left[Q\left[\theta_{k}^{n}\right]_{X_{k+1}^{i, n}, x} Q\left[\theta_{k}^{n}\right]_{X_{k+1}^{j, n}, y} \mid \mathcal{F}_{k}^{n}\right]=\left(K\left[\theta_{k}^{n}\right] Q\left[\theta_{k}^{n}\right]\right)_{X_{k}^{i, n}, x}\left(K\left[\theta_{k}^{n}\right] Q\left[\theta_{k}^{n}\right]\right)_{X_{k}^{j, n}, y} .
$$

Therefore, for each $x, y \in \Delta^{o}$,

$$
\begin{aligned}
& \mathbf{E}\left[e_{k+1}^{n}(x) e_{k+1}^{n}(y) \mid \mathcal{F}_{k}^{n}\right] \\
&=\frac{1}{a(n)^{2}} \sum_{i, j=1}^{a(n)} \mathbf{E}\left[Q\left[\theta_{k}^{n}\right]_{X_{k+1}^{i, n}, x} Q\left[\theta_{k}^{n}\right]_{X_{k+1}^{j, n}, y}-Q\left[\theta_{k}^{n}\right]_{X_{k+1}^{i, n}, x}\left(K\left[\theta_{k}^{n}\right] Q\left[\theta_{k}^{n}\right]\right)_{X_{k}^{j, n}, y}\right. \\
&\left.\quad-Q\left[\theta_{k}^{n}\right]_{X_{k+1}^{j, n}, y}\left(K\left[\theta_{k}^{n}\right] Q\left[\theta_{k}^{n}\right]\right)_{X_{k}^{i, n}, x}+\left(K\left[\theta_{k}^{n}\right] Q\left[\theta_{k}^{n}\right]\right)_{X_{k}^{i, n}, x}\left(K\left[\theta_{k}^{n}\right] Q\left[\theta_{k}^{n}\right]\right)_{X_{k}^{j, n}, y} \mid \mathcal{F}_{k}^{n}\right] \\
&=\frac{1}{a(n)^{2}} \sum_{i, j=1}^{a(n)} \mathbf{E}\left[Q\left[\theta_{k}^{n}\right]_{X_{k+1}^{i, n}, x} Q\left[\theta_{k}^{n}\right]_{X_{k+1}^{j, n}, y}-\left(K\left[\theta_{k}^{n}\right] Q\left[\theta_{k}^{n}\right]\right)_{X_{k}^{i, n}, x}\left(K\left[\theta_{k}^{n}\right] Q\left[\theta_{k}^{n}\right]\right)_{X_{k}^{j, n}, y} \mid \mathcal{F}_{k}^{n}\right] \\
&=\frac{1}{a(n)^{2}} \sum_{i=1}^{a(n)}\left(\sum_{w \in \Delta^{o}} K\left[\theta_{k}^{n}\right]_{X_{k}^{i, n}, w} Q\left[\theta_{k}^{n}\right]_{w, x} Q\left[\theta_{k}^{n}\right]_{w, y}-\left(K\left[\theta_{k}^{n}\right] Q\left[\theta_{k}^{n}\right]\right)_{X_{k}^{i, n}, y}\left(K\left[\theta_{k}^{n}\right] Q\left[\theta_{k}^{n}\right]\right)_{X_{k}^{i, n}, x}\right),
\end{aligned}
$$

where in the last line we have used (3.4) and (3.5). Recalling the definition of $F_{\theta}(\cdot)$ we now see that

$$
\mathbf{E}\left[e_{k+1}^{n}(x) e_{k+1}^{n}(y) \mid \mathcal{F}_{k}^{n}\right]=\frac{1}{a(n)^{2}} \sum_{i=1}^{a(n)} F_{\theta_{k}^{n}}\left(X_{k}^{i, n}\right)_{x, y}
$$

We can write

$$
F_{\theta_{k}^{n}}\left(X_{k}^{i, n}\right)_{x, y}=\left(U_{*}\right)_{x, y}+\left(D_{k}^{(1), i, n}\right)_{x, y}+\left(D_{k}^{(2), i, n}\right)_{x, y},
$$

where

$$
D_{k}^{(1), i, n} \doteq \sum_{w \in \Delta^{o}}\left(F_{\theta_{k}^{n}}(w) \pi\left(\theta_{k}^{n}\right)_{w}-F_{\theta_{*}}(w)\left(\theta_{*}\right)_{w}\right), \quad D_{k}^{(2), i, n} \doteq F_{\theta_{k}^{n}}\left(X_{k}^{i, n}\right)-\sum_{w \in \Delta^{o}} F_{\theta_{k}^{n}}(w) \pi\left(\theta_{k}^{n}\right)_{w}
$$

Letting

$$
D_{k}^{(1), n} \doteq \frac{1}{a(n)} \sum_{i=1}^{a(n)} D_{k}^{(1), i, n}=\sum_{w \in \Delta^{o}}\left(F_{\theta_{k}^{n}}(w) \pi\left(\theta_{k}^{n}\right)_{w}-F_{\theta_{*}}(w)\left(\theta_{*}\right)_{w}\right)
$$




$$
D_{k}^{(2), n} \doteq \frac{1}{a(n)} \sum_{i=1}^{a(n)} D_{k}^{(2), i, n}
$$

and using (3.7), we obtain the identity in (3.3).

Proof of Claim (i): Since $\pi\left(\theta_{*}\right)=\theta_{*}$, we have that

$$
D_{k}^{(1), n}=\sum_{w \in \Delta^{o}}\left(F_{\theta_{k}^{n}}(w) \pi\left(\theta_{k}^{n}\right)_{w}-F_{\theta_{*}}(w) \pi\left(\theta_{*}\right)_{w}\right)
$$

Since the maps $\theta \mapsto K[\theta], \theta \mapsto Q[\theta]$ are bounded and Lipschitz, it follows that $\theta \mapsto F_{\theta}(w)$ is a bounded and Lipschitz map as well for every $w \in \Delta^{o}$. Also, $\theta \mapsto \pi(\theta)$ is a bounded and Lipschitz map (see e.g. [6], Cor. 2.3). Combining these facts we see that $\theta \mapsto \sum_{w \in \Delta^{o}} F_{\theta}(w) \pi(\theta)_{w}$ is a bounded and Lipschitz map as well. The claim in (i) is now immediate from the representation of $D_{k}^{(1), n}$ in (3.8).

Proof of Claim (ii): It suffices to show that for each $(u, v) \in \Delta^{o} \times \Delta^{o}$, there are some $C_{2}, \beta>0$ such that for all $n \in \mathbb{N}$ and $1 \leq k \leq n$,

$$
\gamma_{k} \mathbf{E}\left\|\sum_{m=1}^{k}\left(D_{m-1}^{(2), n}\right)_{u, v}\right\| \leq C_{2} k^{-\beta}
$$

Now fix $(u, v) \in \Delta^{o} \times \Delta^{o}$ and, abusing notation, denote $\left(D_{m}^{(2), n}\right)_{u, v}$ once more as $D_{m}^{(2), n}$. By another abuse of notation, denote the $(u, v)$-th coordinate of $F_{\theta}(x)$, for $\theta \in \mathcal{P}\left(\Delta^{o}\right)$ and $x \in \Delta^{o}$, by $F_{\theta}(x)$ as well. For $\theta \in \mathcal{P}\left(\Delta^{o}\right)$ let $U_{\theta} \in \mathbb{R}^{d}$ be the vector whose $x$-th coordinate is given by $U_{\theta}(x) \doteq\left(Q[\theta] F_{\theta}\right)(x)$. By the Poisson equation (1.16) we have that

$$
\left[(I-K[\theta]) U_{\theta}\right](x)=F_{\theta}(x)-\sum_{w \in \Delta^{o}} F_{\theta}(w) \pi(\theta)_{w}
$$

Therefore, if we let

$$
D_{k}^{(2, a), i, n} \doteq U_{\theta_{k}^{n}}\left(X_{k+1}^{i, n}\right)-\left(K\left[\theta_{k}^{n}\right] U_{\theta_{k}^{n}}\right)\left(X_{k}^{i, n}\right), \quad D_{k}^{(2, b), i, n} \doteq U_{\theta_{k}^{n}}\left(X_{k}^{i, n}\right)-U_{\theta_{k}^{n}}\left(X_{k+1}^{i, n}\right)
$$

and

$$
D_{k}^{(2, a), n} \doteq \frac{1}{a(n)} \sum_{i=1}^{a(n)} D_{k}^{(2, a), i, n}, \quad D_{k}^{(2, b), n} \doteq \frac{1}{a(n)} \sum_{i=1}^{a(n)} D_{k}^{(2, b), i, n}
$$

then $D_{k}^{(2), n}=D_{k}^{(2, a), n}+D_{k}^{(2, b), n}$. Note that with $\mathcal{G}_{k}^{n} \doteq \mathcal{F}_{k+1}^{n}$, we have that, for each fixed $n \in \mathbb{N},\left\{D_{k}^{(2, a), n}\right\}_{k=1}^{\infty}$ is a $\mathcal{G}_{k}^{n}$-martingale increment sequence. Applying Burkholder's inequality, we see that, for some $\kappa_{1} \in(0, \infty)$, and for all $n \in \mathbb{N}$,

$$
\mathbf{E}\left[\left|\sum_{m=1}^{k} D_{m-1}^{(2, a), n}\right|^{2}\right] \leq \kappa_{1} \sum_{m=1}^{k} \mathbf{E}\left|D_{m-1}^{(2, a), n}\right|^{2} \leq \kappa_{1}\left(\frac{1}{a(n)}\right)^{2} \sum_{m=1}^{k} \sum_{i, j=1}^{a(n)} \mathbf{E}\left[D_{m-1}^{(2, a), i, n} D_{m-1}^{(2, a), j, n}\right] .
$$


For $i \neq j$, we have by a conditioning argument, and using (1.3), that

$$
\mathbf{E}\left[D_{m}^{(2, a), i, n} D_{m}^{(2, a), j, n}\right]=0
$$

Let

$$
\kappa_{2} \doteq \sup _{\theta \in \mathcal{P}\left(\Delta^{o}\right), x, y \in \Delta^{o}}\left|U_{\theta}(x)-\left(K[\theta] U_{\theta}\right)(y)\right|^{2}<\infty
$$

Then

$$
\mathbf{E}\left[\left(D_{m}^{(2, a), i, n}\right)^{2}\right]=\mathbf{E}\left[\left(U_{\theta_{m}^{n}}\left(X_{m+1}^{i, n}\right)-\left(K\left[\theta_{m}^{n}\right] U_{\theta_{m}^{n}}\right)\left(X_{m}^{i, n}\right)\right)^{2}\right] \leq \kappa_{2}
$$

Combining (3.9), (3.10), and (3.11) we see that with $\kappa_{3}=\kappa_{1} \kappa_{2}$,

$$
\mathbf{E}\left[\left|\sum_{m=1}^{k} D_{m-1}^{(2, a), n}\right|^{2}\right] \leq \kappa_{1} \sum_{m=1}^{k} \frac{1}{a(n)^{2}} \sum_{i=1}^{a(n)} \mathbf{E}\left[\left(D_{m-1}^{(2, a), i, n}\right)^{2}\right] \leq \kappa_{3} \frac{k}{a(n)}
$$

Applying the Cauchy-Schwarz inequality, we have, for some $\kappa_{4} \in(0, \infty)$, and all $n \in \mathbb{N}, 0 \leq k \leq n$,

$$
\gamma_{k} \mathbf{E}\left|\sum_{m=1}^{k} D_{m-1}^{(2, a), n}\right| \leq \kappa_{3}^{1 / 2} \gamma_{k}\left(\frac{k}{a(n)}\right)^{1 / 2}=\left(\kappa_{4} \frac{\gamma_{k}}{a(n)}\right)^{1 / 2}
$$

We now consider $\left\{D_{k}^{(2, b), n}\right\}$. Letting $\kappa_{5} \doteq \sup _{\theta \in \mathcal{P}\left(\Delta^{\circ}\right), z \in \Delta^{\circ}}\left|U_{\theta}(z)\right|<\infty$,

$$
\begin{aligned}
\left|\sum_{m=1}^{k} D_{m-1}^{(2, b), n}\right| & =\left|\sum_{m=1}^{k} \frac{1}{a(n)} \sum_{i=1}^{a(n)} D_{m-1}^{(2, b), i, n}\right| \\
& =\left|\frac{1}{a(n)} \sum_{i=1}^{a(n)} \sum_{m=1}^{k}\left(U_{\theta_{m-1}^{n}}\left(X_{m-1}^{i, n}\right)-U_{\theta_{m-1}^{n}}\left(X_{m}^{i, n}\right)\right)\right| \\
& =\left|\frac{1}{a(n)} \sum_{i=1}^{a(n)}\left(U_{\theta_{0}^{n}}\left(X_{0}^{i, n}\right)-U_{\theta_{k-1}^{n}}\left(X_{k}^{i, n}\right)+\sum_{m=1}^{k}\left(U_{\theta_{m}^{n}}\left(X_{m}^{i, n}\right)-U_{\theta_{m-1}^{n}}\left(X_{m}^{i, n}\right)\right)\right)\right| \\
& \leq 2 \kappa_{5}+\frac{1}{a(n)} \sum_{i=1}^{a(n)}\left|\sum_{m=1}^{k}\left(U_{\theta_{m}^{n}}\left(X_{m}^{i, n}\right)-U_{\theta_{m-1}^{n}}\left(X_{m}^{i, n}\right)\right)\right|
\end{aligned}
$$

Since the maps $\theta \mapsto K[\theta]$ and $\theta \mapsto Q[\theta]$ are bounded Lipschitz maps, there is a $\kappa_{6} \in(0, \infty)$ such that for all $x \in \Delta^{o}$ and $\theta, \theta^{\prime} \in \mathcal{P}\left(\Delta^{o}\right),\left|U_{\theta}(x)-U_{\theta^{\prime}}(x)\right| \leq \kappa_{6}\left\|\theta-\theta^{\prime}\right\|$. Observe that

$$
\left\|\theta_{m}^{n}-\theta_{m-1}^{n}\right\|=\gamma_{m}\left\|a(n)^{-1} \sum_{i=1}^{a(n)} \delta_{X_{m}^{i, n}}-\theta_{m-1}^{n}\right\| \leq 2 \gamma_{m},
$$


so for some $\kappa_{7} \in(0, \infty)$,

$$
\left|\sum_{m=1}^{k} D_{m-1}^{(2, b), n}\right| \leq\left(2 \kappa_{5}+\frac{\kappa_{6}}{a(n)} \sum_{i=1}^{a(n)} \sum_{m=1}^{k}\left\|\theta_{m}^{n}-\theta_{m-1}^{n}\right\|\right) \leq \kappa_{7}\left(1+\sum_{m=1}^{k} \gamma_{m}\right) .
$$

Combining (3.12) and (3.14) we see that for some $\kappa_{8} \in(0, \infty)$,

$$
\gamma_{k} \mathbf{E}\left\|\sum_{m=1}^{k} D_{m-1}^{(2), n}\right\| \leq \kappa_{8}\left[\left(\frac{\gamma_{k}}{a(n)}\right)^{1 / 2}+\gamma_{k} \sum_{m=1}^{k} \gamma_{m}\right]
$$

The result follows.

\subsection{Bounds on the moments of the error terms}

The following result gives a useful moment bound for $\left\{e_{k}^{n}\right\}$.

Proposition 3.2. There exists $C \in(0, \infty)$ such that for all $n \in \mathbb{N}$ and $0 \leq k \leq n-1$,

$$
\mathbf{E}\left\|e_{k+1}^{n}\right\|^{4} \leq \frac{C}{a(n)^{2}}
$$

Proof. Recall that

$$
e_{k+1}^{n}=\frac{1}{\gamma_{k+1}} \delta_{k+1}^{1, n}=\frac{1}{a(n)} \sum_{i=1}^{a(n)} \xi_{i}
$$

where for each $1 \leq i \leq a(n)$ and $x \in \Delta^{o}, \xi_{i}(x) \doteq Q\left[\theta_{k}^{n}\right]_{X_{k+1}^{i, n}, x}-\left(K\left[\theta_{k}^{n}\right] Q\left[\theta_{k}^{n}\right]\right)_{X_{k}^{i, n}, x}$. The result is now immediate on observing that if $1 \leq i_{1}, i_{2}, i_{3}, i_{4} \leq a(n)$ and $i_{4} \notin\left\{i_{1}, i_{2}, i_{3}\right\}$, then we have that $\mathbf{E}\left[\xi_{i_{1}}(x) \xi_{i_{2}}(x) \xi_{i_{3}}(y) \xi_{i_{4}}(y)\right]=0$, and, for $x, y \in \Delta^{o}, 1 \leq i_{1}, i_{2}, i_{3}, i_{4} \leq a(n), 0 \leq k \leq n-1$ and $n \in \mathbb{N}$,

$$
\left|\xi_{i_{1}}(x) \xi_{i_{2}}(x) \xi_{i_{3}}(y) \xi_{i_{4}}(y)\right| \leq\left(\sup _{\theta \in \mathcal{P}\left(\Delta^{\circ}\right)} \sup _{\substack{x_{1}, y_{1} \\ x_{2}, y_{2}}}\left|Q\left[\theta \Delta_{\Delta^{\circ}} \mid\right]_{x_{1}, y_{1}}-(K[\theta] Q[\theta])_{x_{2}, y_{2}}\right|\right)^{4}<\infty
$$

\subsection{Analysis of the remainder terms}

In this section we provide bounds to control the remainder terms $r_{k+1}^{n}$.

Proposition 3.3. We can write $r_{k+1}^{n}=r_{k+1}^{n, a}+r_{k+1}^{n, b}$, such that for some $C \in(0, \infty)$, and all $n \in \mathbb{N}$ and $0 \leq k \leq n-1$,
(a) $\mathbf{E}\left\|\frac{1}{\gamma_{k+1}} r_{k+1}^{n, a}\right\| \leq C$
(b) $\left\|\sum_{i=k}^{n-1} r_{i+1}^{n, b}\right\| \leq C$. 
Proof. Recall that

$$
r_{k+1}^{n} \doteq \frac{1}{\gamma_{k+1}}\left(\delta_{k+1}^{2, n}+\delta_{k+1}^{3, n}+\delta_{k+1}^{4, n}\right)=\frac{1}{a(n)} \sum_{j=1}^{a(n)}\left(\left(K\left[\theta_{k}^{n}\right] Q\left[\theta_{k}^{n}\right]\right)_{X_{k}^{j, n}, \cdot}-\left(K\left[\theta_{k}^{n}\right] Q\left[\theta_{k}^{n}\right]\right)_{X_{k+1}^{j, n}, .}\right)
$$

Rewrite this as $r_{k+1}^{n}=r_{k+1}^{n, a}+r_{k+1}^{n, b}$, where

$$
r_{k+1}^{n, a} \doteq \frac{1}{a(n)} \sum_{j=1}^{a(n)}\left(\left(K\left[\theta_{k+1}^{n}\right] Q\left[\theta_{k+1}^{n}\right]\right)_{X_{k+1}^{j, n}, \cdot}-\left(K\left[\theta_{k}^{n}\right] Q\left[\theta_{k}^{n}\right]\right)_{X_{k+1}^{j, n}, \cdot}\right)
$$

and

$$
r_{k+1}^{n, b} \doteq \frac{1}{a(n)} \sum_{j=1}^{a(n)}\left(\left(K\left[\theta_{k}^{n}\right] Q\left[\theta_{k}^{n}\right]\right)_{X_{k}^{j, n}, \cdot}-\left(K\left[\theta_{k+1}^{n}\right] Q\left[\theta_{k+1}^{n}\right]\right)_{X_{k+1}^{j, n}, \cdot}\right)
$$

Proof of Claim (a): Since $\theta \mapsto K[\theta], \theta \mapsto Q[\theta]$ are bounded Lipschitz maps, there is a $\kappa_{1} \in(0, \infty)$ such that for all $\theta, \theta^{\prime} \in \mathcal{P}\left(\Delta^{o}\right)\left\|K[\theta] Q[\theta]-K\left[\theta^{\prime}\right] Q\left[\theta^{\prime}\right]\right\| \leq \kappa_{1}\left\|\theta-\theta^{\prime}\right\|$. From this and (3.13) it follows that

$$
\left\|r_{k+1}^{n, a}\right\| \leq \frac{\kappa_{1}}{a(n)} \sum_{j=1}^{a(n)}\left\|\theta_{k+1}^{n}-\theta_{k}^{n}\right\|=\kappa_{1}\left\|\theta_{k+1}^{n}-\theta_{k}^{n}\right\| \leq 2 \kappa_{1} \gamma_{k+1}
$$

which shows that $\mathbf{E}\left\|\gamma_{k+1}^{-1} r_{k+1}^{n, a}\right\| \leq 2 \kappa_{1}$.

Proof of Claim (b): From the definition of $r_{i}^{n, b}$, Note that

$$
\sum_{i=k}^{n-1} r_{i+1}^{n, b}=\frac{1}{a(n)} \sum_{j=1}^{a(n)}\left(\left(K\left[\theta_{k}^{n}\right] Q\left[\theta_{k}^{n}\right]\right)_{X_{k}^{j, n}, \cdot}-\left(K\left[\theta_{n}^{n}\right] Q\left[\theta_{n}^{n}\right]\right)_{X_{n}^{j, n}, \cdot}\right)
$$

from which it follows that

$$
\left\|\sum_{i=k}^{n-1} r_{i+1}^{n, b}\right\| \leq \kappa_{2} \doteq 2 \sup _{\theta \in \mathcal{P}\left(\Delta^{o}\right)} \sup _{x \in \Delta^{\circ}}\left\|(K[\theta] Q[\theta])_{x, \cdot}\right\| .
$$

\section{Central limit theorem for Algorithm I}

The goal of this section is to prove Theorem 1.3. To do this we first study the linearized evolution (1.20). Then, in Section 4.1, we study the asymptotic behavior of the discrepancy (1.21). Finally, in Section 4.3 we present the proof of Theorem 1.3. 


\subsection{The linearized evolution}

Let, for $1 \leq k \leq m<\infty$,

$$
\psi_{*}(m, k) \doteq \prod_{j=k}^{m}\left(I+\gamma_{j} \nabla h\left(\theta_{*}\right)\right), \quad \psi_{*}(m, m+1) \doteq I .
$$

Then by a simple recursion we see that for $0 \leq m \leq n-1$,

$$
\mu_{m+1}^{n}=\sum_{k=1}^{m+1} \gamma_{k} \psi_{*}(m+1, k+1) e_{k}^{n}
$$

Furthermore, from Lemma 5.8 of [21], with $L$ as introduced above (1.2), for each $0<L^{\prime}<L$ there is a $C\left(L^{\prime}\right) \in$ $(0, \infty)$ such that for all $n \in \mathbb{N}$ and $1 \leq k \leq n$

$$
\left\|\psi_{*}(n, k)\right\| \leq C\left(L^{\prime}\right) \exp \left(-L^{\prime} \sum_{j=k}^{n} \gamma_{j}\right)
$$

The following proposition provides some useful bounds on $\left\{\mu_{k}^{n}\right\}$.

Proposition 4.1. The following hold:

(i) With probability one, as $n \rightarrow \infty$ we have $\mu_{n}^{n} \rightarrow 0$. Furthermore, for each $p \in(0,1)$, and a.e. $\omega$, there is some $\alpha>0$ and $n_{0}(\omega) \in \mathbb{N}$ such that if $n \geq n_{0}(\omega)$ and $n^{p} \leq k \leq n$, then $\left\|\mu_{k+1}^{n}\right\| \leq k^{-\alpha}$.

(ii) Suppose that $\gamma_{*}>(2 L)^{-1}$. Then there is some $C>0$ such that for all $n \in \mathbb{N}$ and $0 \leq k \leq n-1$,

$$
\mathbf{E}\left\|\mu_{k+1}^{n}\right\|^{2} \leq \frac{C \gamma_{k+1}}{a(n)}
$$

Proof. The proof of (i) is similar to the proof of Theorem 1.2 and is omitted for brevity.

Next, using (4.2), (4.3), and Proposition 3.2, we see that for each $L^{\prime} \in(0, L)$, there is a $\kappa_{1}\left(L^{\prime}\right) \in(0, \infty)$, such that, for $0 \leq m \leq n-1$,

$$
\mathbf{E}\left[\left\|\mu_{m+1}^{n}\right\|^{2}\right] \leq \sum_{k=1}^{m+1} \gamma_{k}^{2}\left\|\psi_{*}(m+1, k+1)\right\|^{2} \mathbf{E}\left[\left\|e_{k}^{n}\right\|^{2}\right] \leq \frac{\kappa_{1}\left(L^{\prime}\right)}{a(n)} \sum_{k=1}^{m+1} \gamma_{k}^{2} \exp \left(-2 L^{\prime} \sum_{j=k+1}^{m+1} \gamma_{j}\right)
$$

Choosing $L^{\prime} \in(0, L)$ such that $L^{\prime} \gamma_{*}>1 / 2$, and using the form of $\gamma_{k}$, we can find a $\kappa_{2} \in(0, \infty)$ such that for all $0 \leq m \leq n-1, \mathbf{E}\left\|\mu_{m+1}^{n}\right\|^{2} \leq \frac{\kappa_{2} \gamma_{m+1}}{a(n)}$. The result follows.

\subsection{Analysis of the discrepancy}

The following result is used to study the asymptotic behavior of $\left\{\rho_{n}^{n}\right\}$. Following [21], consider for $\theta \in \mathcal{P}\left(\Delta^{o}\right)$ the collection of $d \times d$ matrices $\left\{\mathbf{R}_{i}^{(n)}(\theta)\right\}_{i=1}^{d}$ defined as

$$
\mathbf{R}_{i}^{(n)}(\theta)[k, l] \doteq \int_{0}^{1} \frac{1}{2}(1-t)^{2} \frac{\partial^{2} h_{i}}{\partial \theta_{k} \partial \theta_{l}}\left(\theta+t\left(\theta-\theta_{*}\right)\right) \mathrm{d} t, \quad 1 \leq k, l \leq d .
$$


For $0 \leq j \leq n$, we denote the random matrix $\mathbf{R}_{i}^{(n)}\left(\theta_{j}^{n}\right)$ as $R_{i}^{(n, j)}$. Then, using Taylor's expansion, for $1 \leq i \leq d$ and $0 \leq j \leq n$,

$$
h_{i}\left(\theta_{j}^{n}\right)=\nabla h_{i}\left(\theta_{*}\right) \cdot\left(\theta_{j}^{n}-\theta_{*}\right)+\left(\theta_{j}^{n}-\theta_{*}\right)^{T} R_{i}^{(n, j)}\left(\theta_{j}^{n}-\theta_{*}\right) .
$$

For brevity we write the above display in a vector form as

$$
h\left(\theta_{j}^{n}\right)=\nabla h\left(\theta_{*}\right)\left(\theta_{j}^{n}-\theta_{*}\right)+\left(\theta_{j}^{n}-\theta_{*}\right)^{T} R_{\bullet}^{(n, j)}\left(\theta_{j}^{n}-\theta_{*}\right) .
$$

Corollary 4.2. Let, for $1 \leq k \leq n<\infty$,

$$
\psi(n, k) \doteq \prod_{j=k}^{n}\left(I+\gamma_{j}\left(\nabla h\left(\theta_{*}\right)+2\left(\mu_{j-1}^{n}\right)^{T} R_{\bullet}^{(n, j-1)}+\left(\rho_{j-1}^{n}\right)^{T} R_{\bullet}^{(n, j-1)}\right)\right) .
$$

Then for each $p \in(0,1), L^{\prime} \in(0, L)$, and a.e. $\omega$, there is a $C=C\left(p, L^{\prime}, \omega\right) \in(0, \infty)$ such that if $n^{p} \leq k \leq n$, then

$$
\|\psi(n, k)\| \leq C \exp \left(-L^{\prime} \sum_{j=k}^{n} \gamma_{j}\right)
$$

Proof. Let $A \doteq \nabla h\left(\theta_{*}\right)$ and

$$
\begin{aligned}
A_{j}^{n} & \doteq \nabla h\left(\theta_{*}\right)+2\left(\mu_{j-1}^{n}\right)^{T} R_{\bullet}^{(n, j-1)}+\left(\rho_{j-1}^{n}\right)^{T} R_{\bullet}^{(n, j-1)} \\
& =\nabla h\left(\theta_{*}\right)+\left(\mu_{j-1}^{n}\right)^{T} R_{\bullet}^{(n, j-1)}+\left(\theta_{j-1}^{n}-\theta_{*}\right)^{T} R_{\bullet}^{(n, j-1)},
\end{aligned}
$$

so that with $\kappa_{1} \doteq \sup _{n \in \mathbb{N}} \sup _{\theta \in \mathcal{P}\left(\Delta^{o}\right)} \max _{1 \leq i \leq d}\left\|R_{i}^{(n)}(\theta)\right\|$, we have

$$
\left\|A_{j}^{n}-A\right\|=\left\|\left(\mu_{j-1}^{n}\right)^{T} R_{\bullet}^{(n, j-1)}+\left(\theta_{j-1}^{n}-\theta_{*}\right)^{T} R_{\bullet}^{(n, j-1)}\right\| \leq \kappa_{1}\left(\left\|\mu_{j-1}^{n}\right\|+\left\|\theta_{j-1}^{n}-\theta_{*}\right\|\right) .
$$

Fix $p \in(0,1)$ and $L^{\prime} \in(0, L)$. Applying Proposition 4.1(i) and Theorem 1.2, choose $\alpha>0$ and, for a.e. $\omega, n_{1} \in \mathbb{N}$ such that if $n \geq n_{1}$, and $n^{p} \leq j \leq n$, then $\left\|\mu_{j-1}^{n}\right\| \leq j^{-\alpha}$ and $\left\|\theta_{j-1}^{n}-\theta_{*}\right\| \leq j^{-\alpha}$. Thus, for a.e. $\omega$ there is an $n_{0} \in \mathbb{N}$ such that for all $n \geq n_{0}$ and $n^{p} \leq j \leq n,\left\|A_{j}^{n}-A\right\| \leq j^{-\alpha / 2}$. The result now follows from Lemma A.1.

Recall that $\sigma_{n}=\sqrt{a(n) / \gamma_{n}}$. The next result will be used to show that $\sigma_{n} \rho_{n}^{n} \stackrel{\mathbf{P}}{\rightarrow} 0$ as $n \rightarrow \infty$.

Lemma 4.3. Suppose that $\gamma_{*}>L^{-1}$ and $a(n) / n \rightarrow 0$ as $n \rightarrow \infty$. Then, for some $\kappa, p \in(0,1)$, we have, as $n \rightarrow \infty$,

$$
\sigma_{n}^{1+\kappa}\left[\rho_{n}^{n}-\sum_{k=n^{p}}^{n} \gamma_{k} \psi(n, k+1) r_{k}^{n}\right] \stackrel{\mathbf{P}}{\rightarrow} 0
$$

and

$$
\sigma_{n}\left[\sum_{k=n^{p}}^{n} \gamma_{k} \psi(n, k+1) r_{k}^{n}\right] \stackrel{\mathbf{P}}{\rightarrow} 0
$$


Proof. Fix $L^{\prime} \in\left(\gamma_{*}^{-1}, L\right)$ and let $p \in\left(0,1-\left(L^{\prime} \gamma_{*}\right)^{-1}\right)$. Using (1.19), (1.20), (1.21), (4.5) and a recursive argument, we can write

$$
\rho_{n}^{n} \doteq \psi\left(n, n^{p}\right) \rho_{n^{p}-1}^{n}+\sum_{k=n^{p}}^{n} \gamma_{k} \psi(n, k+1)\left[r_{k}^{n}+\left(\mu_{k-1}^{n}\right)^{T} R_{\bullet}^{(n, k-1)} \mu_{k-1}^{n}\right]
$$

or equivalently,

$$
\rho_{n}^{n}-\sum_{k=n^{p}}^{n} \gamma_{k} \psi(n, k+1) r_{k}^{n}=\psi\left(n, n^{p}\right) \rho_{n^{p}-1}^{n}+\sum_{k=n^{p}}^{n} \gamma_{k} \psi(n, k+1)\left(\mu_{k-1}^{n}\right)^{T} R_{\bullet}^{(n, k-1)} \mu_{k-1}^{n} .
$$

Fix $\kappa \in\left(0, \frac{(1-p) L^{\prime} \gamma_{*}-1}{2} \wedge \frac{1}{4}\right)$. We begin by showing that

$$
\sigma_{n}^{1+\kappa} \psi\left(n, n^{p}\right) \rho_{n^{p}-1}^{n} \stackrel{\mathbf{P}}{\rightarrow} 0 .
$$

Since $\left\|\rho_{n^{p}-1}^{n}\right\|$ is a bounded sequence, it is enough to show that $\sigma_{n}^{1+\kappa} \psi\left(n, n^{p}\right)$ converges to 0 in probability. From Corollary 4.2 , for a.e. $\omega$, there is a $\kappa_{1}(\omega) \in(0, \infty)$ such that

$$
\sigma_{n}^{1+\kappa}\left\|\psi\left(n, n^{p}\right)\right\| \leq \kappa_{1}(a(n) n)^{\frac{1}{2}(1+\kappa)} \exp \left(-L^{\prime} \sum_{j=n^{p}}^{n} \gamma_{j}\right)=\kappa_{1}\left(\frac{a(n)}{n}\right)^{\frac{1}{2}(1+\kappa)} n^{1+\kappa} \exp \left(-L^{\prime} \sum_{j=n^{p}}^{n} \gamma_{j}\right)
$$

From the definition of $\gamma_{k}$ we see that for all $1 \leq k \leq n$

$$
\exp \left(-L^{\prime} \sum_{j=k}^{n} \gamma_{j}\right) \leq\left(\frac{N_{*}+k}{N_{*}+n}\right)^{L^{\prime} \gamma_{*}}
$$

From our choice of $\kappa,(p-1) L^{\prime} \gamma_{*}+1+\kappa<0$, and so we have, on applying (4.10) with $k=n^{p}$, that the expression in (4.9) converges to 0 . This completes the proof of (4.8). We now show that

$$
\sigma_{n}^{1+\kappa} \sum_{k=n^{p}}^{n} \gamma_{k} \psi(n, k+1)\left(\mu_{k-1}^{n}\right)^{T} R_{\bullet}^{(n, k-1)} \mu_{k-1}^{n} \stackrel{\mathbf{P}}{\rightarrow} 0 .
$$

Using the uniform-boundedness of $\left\{R_{\bullet}^{(n, k-1)}\right\}$ and Corollary 4.2, for a.e. $\omega$, we can find a $\kappa_{2}(\omega) \in(0, \infty)$ such that

$$
\begin{aligned}
\left\|\sum_{k=n^{p}}^{n} \gamma_{k} \psi(n, k+1)\left(\mu_{k-1}^{n}\right)^{T} R_{\bullet}^{(n, k-1)} \mu_{k-1}^{n}\right\| & \leq \sum_{k=n^{p}}^{n} \gamma_{k}\|\psi(n, k+1)\|\left\|\mu_{k-1}^{n}\right\|^{2}\left\|R_{\bullet}^{(n, k-1)}\right\| \\
& \leq \kappa_{2} \sum_{k=n^{p}}^{n} \gamma_{k} \exp \left(-L^{\prime} \sum_{j=k}^{n} \gamma_{j}\right)\left\|\mu_{k-1}^{n}\right\|^{2} .
\end{aligned}
$$

From Proposition 4.1, there is a $\kappa_{3} \in(0, \infty)$ such that for all $n \in \mathbb{N}$ and $k \leq n, \mathbf{E}\left\|\mu_{k-1}^{n}\right\|^{2} \leq \kappa_{3} \gamma_{k} / a(n)$. It follows that, for some $\kappa_{4} \in(0, \infty)$, with $\tilde{\kappa} \doteq \frac{1}{2}(1+\kappa)<1$, 


$$
\begin{aligned}
\sigma_{n}^{1+\kappa} \mathbf{E}\left(\sum_{k=n^{p}}^{n} \gamma_{k} \exp \left(-L^{\prime} \sum_{j=k}^{n} \gamma_{j}\right)\left\|\mu_{k-1}^{n}\right\|^{2}\right) \\
\quad \leq \kappa_{3} \sigma_{n}^{1+\kappa} \sum_{k=n^{p}}^{n} \gamma_{k} \exp \left(-L^{\prime} \sum_{j=k}^{n} \gamma_{j}\right) \frac{\gamma_{k}}{a(n)} \leq \kappa_{4} n^{\tilde{\kappa}} a(n)^{\tilde{\kappa}-1} \sum_{k=n^{p}}^{n} \gamma_{k}^{2} \exp \left(-L^{\prime} \sum_{j=k}^{n} \gamma_{j}\right) .
\end{aligned}
$$

Using (4.10) once more, we can find some $\kappa_{5} \in(0, \infty)$ such that the last expression is bounded above by

$$
a(n)^{\tilde{\kappa}-1}\left(n+N_{*}\right)^{\tilde{\kappa}-L^{\prime} \gamma_{*}} \sum_{k=n^{p}}^{n}\left(k+N_{*}\right)^{L^{\prime} \gamma_{*}-2}
$$

which tends to 0 as $n \rightarrow \infty$, since $\tilde{\kappa}<1$. Combining this convergence with (4.12) and (4.13), we have (4.11), which together with (4.8) proves the first statement in the lemma.

We now prove the second statement. Let $r_{i}^{n, a}, r_{i}^{n, b}$ be as in (3.15) and (3.16), respectively, so that $r_{i}^{n}=$ $r_{i}^{n, a}+r_{i}^{n, b}$. Using Corollary 4.2, we can find, for a.e. $\omega$, some $\kappa_{6} \equiv \kappa_{6}(\omega) \in(0, \infty)$ such that

$$
\left\|\sum_{k=n^{p}}^{n} \gamma_{k} \psi(n, k+1) r_{k}^{n, a}\right\| \leq \kappa_{6} \sum_{k=n^{p}}^{n} \gamma_{k}^{2} \exp \left(-L^{\prime} \sum_{j=k}^{n} \gamma_{j}\right)\left\|\frac{1}{\gamma_{k}} r_{k}^{n, a}\right\| .
$$

Using Proposition 3.3(a), we can find some $\kappa_{7} \in(0, \infty)$ such that for all $n \in \mathbb{N}$ and $1 \leq k \leq n, \mathbf{E}\left\|r_{k}^{n, a} / \gamma_{k}\right\| \leq \kappa_{7}$. Then, for some $\kappa_{8} \in(0, \infty)$,

$$
\begin{aligned}
\sigma_{n} \sum_{k=n^{p}}^{n} \gamma_{k}^{2} \exp \left(-L^{\prime} \sum_{j=k}^{n} \gamma_{j}\right) \mathbf{E}\left\|\frac{1}{\gamma_{k}} r_{k}^{n, a}\right\| & \leq \kappa_{7} \sigma_{n} \sum_{k=n^{p}}^{n} \gamma_{k}^{2} \exp \left(-L^{\prime} \sum_{j=k}^{n} \gamma_{j}\right) \\
& \leq \kappa_{8} \sqrt{\frac{a(n)}{n} n} \sum_{k=n^{p}}^{n} \gamma_{k}^{2} \exp \left(-L^{\prime} \sum_{j=k}^{n} \gamma_{j}\right)
\end{aligned}
$$

As in (4.14), the last term can be bounded above by

$$
\kappa_{8} \sqrt{\frac{a(n)}{n}} n \frac{\gamma_{*}^{2}}{\left(n+N_{*}\right)^{L^{\prime} \gamma_{*}}} \sum_{k=n^{p}}^{n}\left(k+N_{*}\right)^{L^{\prime} \gamma_{*}-2}
$$

which, since $a(n)=o(n)$, converges to 0 as $n \rightarrow \infty$. Combining (4.15) and (4.16), we have that, as $n \rightarrow \infty$,

$$
\sigma_{n}\left\|\sum_{k=n^{p}}^{n} \gamma_{k} \psi(n, k+1) r_{k}^{n, a}\right\| \stackrel{\mathbf{P}}{\rightarrow} 0 .
$$

Now, consider the term $\sigma_{n}\left\|\sum_{k=1}^{n} \gamma_{k} \psi(n, k+1) r_{k}^{n, b}\right\|$. Define for $n \in \mathbb{N}$ and $1 \leq k \leq n$

$$
\Xi_{k}^{n} \doteq \sum_{i=1}^{k} r_{i}^{n, b}, \quad H_{k}^{n} \doteq \nabla h\left(\theta_{*}\right)+2\left(\mu_{k}^{n}\right)^{T} R_{\bullet}^{(n, k)}+\left(\rho_{k}^{n}\right)^{T} R_{\bullet}^{(n, k)}
$$


and apply the summation by parts formula to see that

$$
\sum_{k=n^{p}}^{n} \gamma_{k} \psi(n, k+1) r_{k}^{n, b}=\gamma_{n} \Xi_{n}^{n}-\gamma_{n^{p}} \psi\left(n, n^{p}+1\right) \Xi_{n^{p}-1}^{n}-\sum_{k=n^{p}}^{n-1} \Xi_{k}^{n}\left(\gamma_{k+1} \psi(n, k+2)-\gamma_{k} \psi(n, k+1)\right) .
$$

Thus

$$
\begin{aligned}
& \sigma_{n} \sum_{k=n^{p}}^{n} \gamma_{k} \psi(n, k+1) r_{k}^{n, b} \\
& =\sigma_{n}\left(\gamma_{n} \Xi_{n}^{n}-\gamma_{n^{p}} \psi\left(n, n^{p}+1\right) \Xi_{n^{p}-1}^{n}\right)-\sigma_{n} \sum_{k=n^{p}}^{n-1} \Xi_{k}^{n}\left(\gamma_{k+1} \psi(n, k+2)-\gamma_{k} \psi(n, k+1)\right) \\
& =\sigma_{n}\left(\gamma_{n} \Xi_{n}^{n}-\gamma_{n^{p}} \psi\left(n, n^{p}+1\right) \Xi_{n^{p}-1}^{n}\right) \\
& \quad-\sigma_{n} \sum_{k=n^{p}}^{n-1} \Xi_{k}^{n}\left(\gamma_{k+1} \psi(n, k+2)-\gamma_{k} \psi(n, k+2)\left(I+\gamma_{k+1} H_{k}^{n}\right)\right) \\
& =\sigma_{n}\left(\gamma_{n} \Xi_{n}^{n}-\gamma_{n^{p}} \psi\left(n, n^{p}+1\right) \Xi_{n^{p}-1}^{n}\right)+\sigma_{n} \sum_{k=n^{p}}^{n-1} \gamma_{k} \gamma_{k+1} \Xi_{k}^{n} \psi(n, k+2)\left(\gamma_{*}^{-1} I+H_{k}^{n}\right) .
\end{aligned}
$$

Applying Corollary 4.2 and Proposition 3.3, for a.e. $\omega$, we can find a $\kappa_{9} \equiv \kappa_{9}(\omega) \in(0, \infty)$ such that

$$
\sigma_{n} \gamma_{n^{p}}\left\|\psi\left(n, n^{p}+1\right) \Xi_{n^{p}-1}^{n}\right\| \leq \kappa_{9} \sigma_{n} \gamma_{n^{p}} \exp \left(-L^{\prime} \sum_{j=n^{p}+1}^{n} \gamma_{j}\right)
$$

Using (4.10), the expression in the previous display can be bounded by

$$
\kappa_{10} \sqrt{n a(n)} \frac{\gamma_{*}}{n^{p}+N_{*}-1}\left(\frac{N_{*}+n^{p}+1}{N_{*}+n}\right)^{L^{\prime} \gamma_{*}}
$$

which tends to 0 as $n \rightarrow \infty$. Also, using Proposition 3.3 we see that for some $\kappa_{11} \in(0, \infty)$

$$
\sigma_{n} \gamma_{n}\left\|\Xi_{n}^{n}\right\| \leq \kappa_{11}\left(\frac{a(n)}{n}\right)^{1 / 2}
$$

which, since $a(n)=o(n)$, also goes to 0 as $n \rightarrow \infty$. Finally, note that

$$
A \doteq \sup _{n \in \mathbb{N}} \sup _{n^{p} \leq k \leq n}\left\|\left(\gamma_{*}^{-1} I+H_{k}^{n}\right)\right\|<\infty \text { a.s. }
$$

which, together with Proposition 3.3(b), ensures that for a.e. $\omega$, there is a $\kappa_{12} \equiv \kappa_{12}(\omega) \in(0, \infty)$ such that

$$
\left\|\sigma_{n} \sum_{k=n^{p}}^{n-1} \gamma_{k} \gamma_{k+1} \Xi_{k}^{n} \psi(n, k+2)\left(\gamma_{*}^{-1} I+H_{k}^{n}\right)\right\| \leq \kappa_{12} \sigma_{n} \sum_{k=n^{p}}^{n-1} \gamma_{k}^{2} \exp \left(-L^{\prime} \sum_{j=k}^{n} \gamma_{j}\right)
$$


which, as for (4.16), goes to 0 as $n \rightarrow \infty$. Upon combining (4.19), (4.20), (4.21), and (4.22), we see that, as $n \rightarrow \infty$,

$$
\sigma_{n}\left\|\sum_{k=n^{p}}^{n} \gamma_{k} \psi(n, k+1) r_{k}^{n, b}\right\| \stackrel{\mathbf{P}}{\rightarrow} 0 .
$$

This, along with (4.17), shows (4.6) and completes the proof of the lemma.

\subsection{Proof of Theorem 1.3}

In order to prove Theorem 1.3, it will be convenient to consider the array $\left\{Z_{n, k}, n \in \mathbb{N}, 1 \leq k \leq n\right\}$ defined for $n \in \mathbb{N}$ and $1 \leq k \leq n$ as

$$
Z_{n, k} \doteq \sigma_{n} \gamma_{k} \psi_{*}(n, k+1) e_{k}^{n}
$$

From (4.2) we see that

$$
\sum_{k=1}^{n} Z_{n, k}=\sigma_{n} \mu_{n}^{n}
$$

The next lemma is used to verify that a conditional Lindeberg condition holds for $\left\{Z_{n, k}\right\}$.

Lemma 4.4. Suppose that $\gamma_{*}>(2 L)^{-1}$. Then, as $n \rightarrow \infty$, we have $\sum_{k=1}^{n} \mathbf{E}\left\|Z_{n, k}\right\|^{4} \rightarrow 0$.

Proof. From Proposition 3.2, there is $\kappa_{1} \in(0, \infty)$ such that for all $n \in \mathbb{N}$ and all $1 \leq k \leq n, \mathbf{E}\left[\left\|e_{k}^{n}\right\|^{4}\right] \leq \frac{\kappa_{1}}{a(n)^{2}}$. Now, fix $L^{\prime} \in(0, L)$ such that $L^{\prime} \gamma_{*}>1 / 2$, and recall from (4.3) that for some $\kappa_{2} \in(0, \infty)$, and for all $n \in \mathbb{N}$ and $1 \leq k \leq n$,

$$
\left\|\psi_{*}(n, k+1)\right\| \leq \kappa_{2} \exp \left(-L^{\prime} \sum_{j=k}^{n} \gamma_{j}\right) .
$$

Thus, for some $\kappa_{3} \in(0, \infty)$ we have that

$$
\begin{aligned}
\sum_{k=1}^{n} \mathbf{E}\left\|Z_{n, k}\right\|^{4} \leq \sum_{k=1}^{n} \sigma_{n}^{4} \gamma_{k}^{4}\left\|\psi_{*}(n, k+1)\right\|^{4} \mathbf{E}\left\|e_{k}^{n}\right\|^{4} \leq \kappa_{3} \sum_{k=1}^{n} \sigma_{n}^{4} \gamma_{k}^{4} \exp \left(-4 L^{\prime} \sum_{j=k}^{n} \gamma_{j}\right) \frac{1}{a(n)^{2}} \\
\leq \kappa_{3} \frac{n^{2} \gamma_{*}^{4}}{\left(N_{*}+n\right)^{4 L^{\prime} \gamma_{*}}} \sum_{k=1}^{n}\left(N_{*}+k\right)^{4\left(L^{\prime} \gamma_{*}-1\right)}
\end{aligned}
$$

which tends to 0 as $n \rightarrow \infty$. The result follows.

The next lemma is used in the proof of Theorem 1.3 to establish the form of the limiting covariance matrix $V$. Recall the matrix $U_{*}$ introduced in (3.2).

Lemma 4.5. Suppose that $\gamma_{*}>(2 L)^{-1}$. Define

$$
V_{n}^{(1)} \doteq \sigma_{n}^{2} \sum_{k=1}^{n} \gamma_{k}^{2} \psi_{*}(n, k+1) \frac{U_{*}}{a(n)} \psi_{*}(n, k+1)^{T},
$$


and

$$
V_{n}^{(2)} \doteq \sigma_{n}^{2} \sum_{k=1}^{n} \gamma_{k}^{2} \psi_{*}(n, k+1)\left(\mathbf{E}\left[e_{k}^{n}\left(e_{k}^{n}\right)^{T} \mid \mathcal{F}_{k-1}^{n}\right]-\frac{U_{*}}{a(n)}\right) \psi_{*}(n, k+1)^{T}
$$

Then $V_{n}^{(1)} \stackrel{\mathbf{P}}{\rightarrow} V$ and $V_{n}^{(2)} \stackrel{\mathbf{P}}{\rightarrow} 0$, where $V$ is the matrix given as the unique solution of the Lyapunov equation

$$
U_{*}+\nabla h\left(\theta_{*}\right) V+V \nabla h\left(\theta_{*}\right)^{T}+\gamma_{*}^{-1} V=0 .
$$

Proof. We begin by noting that the Lyapunov equation in (4.26) has a unique solution. Indeed, note that $U_{*}$ is nonnegative definite and $\nabla h\left(\theta_{*}\right)+\left(2 \gamma_{*}\right)^{-1} I$ is Hurwitz, as $-L+\left(2 \gamma_{*}\right)^{-1}<0$. The unique solvability of (4.26) is now an immediate consequence of Theorem 2.2.3 in [24]. Next, noting that

$$
V_{n}^{(1)}=\gamma_{n}^{-1} \sum_{k=1}^{n} \gamma_{k}^{2} \psi_{*}(n, k+1) U_{*} \psi_{*}(n, k+1)^{T}
$$

the proof that $V_{n}^{(1)} \stackrel{\mathrm{P}}{\rightarrow} V$ as $n \rightarrow \infty$ follows from [21] (see Sect. 5.4, Limiting Variance, therein). Now, recall that with the matrices $\left\{D_{k}^{(1), n}\right\}$ and $\left\{D_{k}^{(2), n}\right\}$ introduced in Proposition 3.1, we can write

$$
\mathbf{E}\left[e_{k}^{n}\left(e_{k}^{n}\right)^{T} \mid \mathcal{F}_{k-1}^{n}\right]-\frac{U_{*}}{a(n)}=\frac{D_{k}^{(1), n}}{a(n)}+\frac{D_{k}^{(2), n}}{a(n)} .
$$

Thus, $V_{n}^{(2)}=V_{n}^{(2, a)}+V_{n}^{(2, b)}$, where

$$
V_{n}^{(2, a)} \doteq \sigma_{n}^{2} \sum_{k=1}^{n} \gamma_{k}^{2} \psi_{*}(n, k+1) \frac{D_{k-1}^{(1), n}}{a(n)} \psi_{*}(n, k+1)^{T}=\frac{1}{\gamma_{n}} \sum_{k=1}^{n} \gamma_{k}^{2} \psi_{*}(n, k+1) D_{k-1}^{(1), n} \psi_{*}(n, k+1)^{T},
$$

and

$$
V_{n}^{(2, b)} \doteq \sigma_{n}^{2} \sum_{k=1}^{n} \gamma_{k}^{2} \psi_{*}(n, k+1) \frac{D_{k-1}^{(2), n}}{a(n)} \psi_{*}(n, k+1)^{T}=\frac{1}{\gamma_{n}} \sum_{k=1}^{n} \gamma_{k}^{2} \psi_{*}(n, k+1) D_{k-1}^{(2), n} \psi_{*}(n, k+1)^{T}
$$

Using part (i) of Proposition 3.1, we can find some $\kappa_{1} \in(0, \infty)$ such that for all $n \in \mathbb{N}$ and $1 \leq k \leq n$, $\left\|D_{k-1}^{(1), n}\right\| \leq \kappa_{1}\left\|\theta_{k-1}^{n}-\theta_{*}\right\|$. Also, for each $p \in(0,1)$, from Theorem 1.2, we can find $\alpha>0$ such that for a.e. $\omega$, there is an $n_{0}(\omega) \in \mathbb{N}$ such that for all $n \geq n_{0}(\omega)$ and $n^{p} \leq k \leq n,\left\|\theta_{k-1}^{n}(\omega)-\theta_{*}\right\| \leq k^{-\alpha}$. Fix $L^{\prime} \in(0, L)$ such 
that $L^{\prime} \gamma_{*}>1 / 2$. Then, for $n \geq n_{0}(\omega)$ and some $\kappa_{2} \in(0, \infty)$, we have

$$
\begin{aligned}
\left\|V_{n}^{(2, a)}\right\| & \leq \frac{1}{\gamma_{n}} \sum_{k=1}^{n} \gamma_{k}^{2}\left\|\psi_{*}(n, k+1)\right\|^{2}\left\|D_{k}^{(1), n}\right\| \\
& \leq \kappa_{2} \frac{1}{\gamma_{n}} \sum_{k=1}^{n} \gamma_{k}^{2} \exp \left(-2 L^{\prime} \sum_{i=k}^{n} \gamma_{i}\right)\left\|\theta_{k}^{n}-\theta_{*}\right\| \\
& =\kappa_{2} \frac{1}{\gamma_{n}}\left(\sum_{k=1}^{n^{p}-1} \gamma_{k}^{2} \exp \left(-2 L^{\prime} \sum_{i=k}^{n} \gamma_{i}\right)\left\|\theta_{k}^{n}-\theta_{*}\right\|+\sum_{k=n^{p}}^{n} \gamma_{k}^{2} \exp \left(-2 L^{\prime} \sum_{i=k}^{n} \gamma_{i}\right)\left\|\theta_{k-1}^{n}-\theta_{*}\right\|\right) \\
& \leq 2 \kappa_{2} \frac{1}{\gamma_{n}}\left(\sum_{k=1}^{n^{p}-1} \gamma_{k}^{2} \exp \left(-2 L^{\prime} \sum_{i=k}^{n} \gamma_{i}\right)+\sum_{k=n^{p}}^{n} \gamma_{k}^{2} \exp \left(-2 L^{\prime} \sum_{i=k}^{n} \gamma_{i}\right) k^{-\alpha}\right) .
\end{aligned}
$$

We can find $\kappa_{3} \in(0, \infty)$ such that first term on the last line is bounded above by

$$
\kappa_{3}\left(n+N_{*}\right)^{-\left(2 L^{\prime} \gamma_{*}-1\right)}\left(n^{p}+N_{*}\right)^{2 L^{\prime} \gamma_{*}-1},
$$

and such that the second term is bounded above by

$$
\kappa_{3} \frac{\left(N_{*}+n\right)}{\left(N_{*}+n\right)^{2 L^{\prime} \gamma_{*}}} \sum_{k=n^{p}}^{n} k^{-\alpha}\left(k+N_{*}\right)^{2 L^{\prime} \gamma_{*}-2}
$$

Since $L^{\prime} \gamma_{*}>1 / 2$, both of these terms converge to 0 as $n \rightarrow \infty$ and so we have that $\left\|V_{n}^{(2, a)}\right\| \rightarrow 0$ almost surely, as $n \rightarrow \infty$. Now, let $\Xi_{k}^{n} \doteq \sum_{j=1}^{k} D_{j-1}^{(2), n}$. Using summation by parts we have

$$
V_{n}^{(2, b)}=\gamma_{n} \Xi_{n}^{n}+\frac{1}{\gamma_{n}} \sum_{k=1}^{n-1}\left(\gamma_{k}^{2} \psi_{*}(n, k+1) \Xi_{k}^{n} \psi_{*}(n, k+1)^{T}-\gamma_{k+1}^{2} \psi_{*}(n, k+2) \Xi_{k}^{n} \psi_{*}(n, k+2)^{T}\right) .
$$

From Proposition 3.1(ii) we have that for some $\kappa_{4}, \beta \in(0, \infty)$ and all $n \in \mathbb{N}, 1 \leq k \leq n, \gamma_{k} \mathbf{E}\left\|\Xi_{k}^{n}\right\| \leq \kappa_{4} k^{-\beta}$, and so

$$
\gamma_{n} \Xi_{n}^{n} \stackrel{\mathbf{P}}{\rightarrow} 0 \text { as } n \rightarrow \infty
$$

Let $\tilde{V}_{n}^{(2, b)}=V_{n}^{(2, b)}-\gamma_{n} \Xi_{n}^{n}$. Following [19], if we let $A_{1} \doteq \gamma_{k} \psi_{*}(n, k+1), A_{2} \doteq \gamma_{k+1} \psi_{*}(n, k+2)$, and $B \doteq \Xi_{k}^{n}$, then using the inequality

$$
\left\|A_{1} B A_{1}^{T}-A_{2} B A_{2}^{T}\right\|=\left\|\left(A_{1}-A_{2}\right) B A_{1}^{T}-A_{2} B\left(A_{2}^{T}-A_{1}^{T}\right)\right\| \leq\left\|A_{1}-A_{2}\right\|\|B\|\left(\left\|A_{1}\right\|+\left\|A_{2}\right\|\right),
$$

we see that

$$
\begin{aligned}
& \left\|\tilde{V}_{n}^{(2, b)}\right\| \\
& \quad \leq \frac{1}{\gamma_{n}} \sum_{k=1}^{n-1}\left(\left\|\gamma_{k} \psi_{*}(n, k+1)-\gamma_{k+1} \psi_{*}(n, k+2)\right\| \cdot\left\|\Xi_{k}^{n}\right\| \cdot\left(\left\|\gamma_{k} \psi_{*}(n, k+1)\right\|+\left\|\gamma_{k+1} \psi_{*}(n, k+2)\right\|\right)\right) .
\end{aligned}
$$


Furthermore, using the fact that $\gamma_{k+1}-\gamma_{k+2} \leq \gamma_{k+1}^{2} / \gamma_{*}$, we can find some $\kappa_{5} \in(0, \infty)$ such that

$$
\begin{aligned}
& \left\|\gamma_{k} \psi_{*}(n, k+1)-\gamma_{k+1} \psi_{*}(n, k+2)\right\|=\left\|\gamma_{k}\left(I+\gamma_{k+1} \nabla h\left(\theta_{*}\right)\right)-\gamma_{k+1} I\right\|\left\|\psi_{*}(n, k+2)\right\| \\
& \leq\left(\gamma_{k}-\gamma_{k+1}+\left\|\nabla h\left(\theta_{*}\right)\right\| \gamma_{k} \gamma_{k+1}\right)\left\|\psi_{*}(n, k+2)\right\| \leq \kappa_{5} \gamma_{k+1}^{2}\left\|\psi_{*}(n, k+2)\right\| .
\end{aligned}
$$

Additionally, there is some $\kappa_{6} \in(0, \infty)$ such that for all $n \in \mathbb{N}$ and $1 \leq k \leq n-1$,

$$
\left\|\gamma_{k} \psi_{*}(n, k+1)\right\|+\left\|\gamma_{k+1} \psi_{*}(n, k+2)\right\| \leq \kappa_{6} \gamma_{k+1}\left\|\psi_{*}(n, k+2)\right\|
$$

Using (4.30), (4.31) and (4.32) we see that

$$
\left\|\tilde{V}_{n}^{(2, b)}\right\| \leq \kappa_{5} \kappa_{6} \frac{1}{\gamma_{n}} \sum_{k=0}^{n-1} \gamma_{k+1}^{2}\left\|\psi_{*}(n, k+2)\right\|^{2} \gamma_{k+1}\left\|\Xi_{k}^{n}\right\| .
$$

Thus, from Proposition 3.1(ii), for some $\kappa_{7}, \kappa_{8} \in(0, \infty)$,

$$
\mathbf{E}\left\|\tilde{V}_{n}^{(2, b)}\right\| \leq \kappa_{7} \frac{1}{\gamma_{n}} \sum_{k=0}^{n-1} \gamma_{k+1}^{2} \exp \left(-2 L^{\prime} \sum_{j=k+2}^{n} \gamma_{j}\right) k^{-\beta} \leq \frac{\kappa_{7} \gamma_{*}}{\left(n+N_{*}\right)^{2 L^{\prime} \gamma_{*}-1}} \sum_{k=0}^{n-1}\left(k+N_{*}\right)^{2\left(L^{\prime} \gamma_{*}-1\right)} k^{-\beta}
$$

which goes to 0 as $n \rightarrow \infty$. Combining the above convergence with (4.29), it follows from (4.28) that $V_{n}^{(2, b)} \stackrel{\mathbf{P}}{\rightarrow} 0$ as $n \rightarrow \infty$. The result follows.

We now complete the proof of Theorem 1.3.

Proof of Theorem 1.3. From (1.21) we see that $\sigma_{n}\left(\theta_{n}^{n}-\theta_{*}\right)=\sigma_{n} \mu_{n}^{n}+\sigma_{n} \rho_{n}^{n}$. Also, from Lemma $4.3, \sigma_{n} \rho_{n}^{n} \stackrel{\mathbf{P}}{\rightarrow} 0$ as $n \rightarrow \infty$. Thus, it suffices to show that $\sigma_{n} \mu_{n}^{n} \stackrel{\mathcal{L}}{\rightarrow} \mathcal{N}(0, V)$ where $V$ is as in the statement of the theorem. Recall the martingale difference array $\left\{Z_{n, k}\right\}$ introduced in (4.23), and note from (4.24) that $\sigma_{n} \mu_{n}^{n}=\sum_{k=1}^{n} Z_{n, k}$. In order to complete the proof we apply ([23], Cor. 3.1). From Lemma 4.4 it follows that for each $\epsilon>0$,

$$
\sum_{k=1}^{n} \mathbf{E}\left[\left\|Z_{n, k}\right\|^{2} \mathbb{1}_{\left\|Z_{n, k}\right\| \geq \epsilon} \mid \mathcal{F}_{k-1}^{n}\right] \stackrel{\mathbf{P}}{\rightarrow} 0
$$

as $n \rightarrow \infty$. Additionally, if we let $\left\{V_{n}^{(1)}\right\},\left\{V_{n}^{(2)}\right\}$ and $V$ be as in Lemma 4.5, then we have from this lemma that

$$
\sum_{k=1}^{n} \mathbf{E}\left[Z_{n, k} Z_{n, k}^{T} \mid \mathcal{F}_{k-1}^{n}\right]=V_{n}^{(1)}+V_{n}^{(2)} \stackrel{\mathbf{P}}{\rightarrow} V
$$

as $n \rightarrow \infty$. Therefore, the conditions of $[23]^{*}$ Corollary 3.1 are satisfied, proving that $\sum_{k=1}^{n} Z_{n, k} \stackrel{\mathcal{L}}{\rightarrow} \mathcal{N}(0, V)$, as $n \rightarrow \infty$. The result follows.

\section{Convergence of Algorithm II}

In this section we prove the a.s. convergence of Algorithm II introduced in Section 1.1. Namely we provide the proof of Theorem 1.4. Recall that in this method, we initialize the scheme with a single particle and as time progresses, particles are added to the system and the total time occupation measure of all particles is used to 
update the SA estimate. The goal of the section is to prove that $\boldsymbol{\theta}_{n} \rightarrow \theta_{*}$ as $n \rightarrow \infty$, where $\boldsymbol{\theta}_{n}$ is as introduced in (1.10). The proof idea is similar to that in [6]. We introduce the continuous-time process $\{\hat{\boldsymbol{\theta}}(t)\}$ given by

$$
\hat{\boldsymbol{\theta}}\left(\tau_{n}+t\right) \doteq \boldsymbol{\theta}_{n}+t \frac{\boldsymbol{\theta}_{n+1}-\boldsymbol{\theta}_{n}}{\tau_{n+1}-\tau_{n}}, \quad t \in\left[0, \gamma_{n+1}\right), n \in \mathbb{N}_{0},
$$

where the sequence $\left\{\tau_{n}\right\}$ is defined in (2.1). As before, for each $\nu \in \mathcal{P}\left(\Delta^{o}\right)$, we denote the solution to the ODE (2.2) by $\left\{\Phi_{t}(\nu)\right\}$. We now recall the notion of an asymptotic pseudo-trajectory for a single trajectory which was introduced first in $[8]$ (see also $[4,6]$ ). Recall the space $\mathcal{C}^{0}$ from Section 1.4.

Definition 5.1. A trajectory $X \in \mathcal{C}^{0}$ is an asymptotic pseudo-trajectory of $\Phi$ if for all $T>0$,

$$
\limsup _{t \rightarrow \infty} \sup _{0 \leq u \leq T}\left\|X(t+u)-\Phi_{u}(X(t))\right\|=0 .
$$

In order to prove Theorem 1.4 we will show that $\{\hat{\boldsymbol{\theta}}\}$ is a.s. an asymptotic pseudo-trajectory of $\Phi$. For this, we begin by decomposing algorithm's noise in the following manner: for each $n \in \mathbb{N}_{0}, 1 \leq i \leq a(n+1)$, and $x \in \Delta^{o}$, let

$$
\delta_{n+1}^{\ell, i}(x) \doteq\left\{\begin{array}{lr}
\gamma_{n+1} Q\left[\boldsymbol{\theta}_{n}\right]_{\boldsymbol{X}_{n+1}^{i}, x}-\gamma_{n+1}\left(K\left[\boldsymbol{\theta}_{n}\right] Q\left[\boldsymbol{\theta}_{n}\right]\right)_{\boldsymbol{X}_{n}^{i}, x} & \ell=1 \\
\gamma_{n+1}\left(K\left[\boldsymbol{\theta}_{n}\right] Q\left[\boldsymbol{\theta}_{n}\right]\right)_{\boldsymbol{X}_{n}^{i}, x}-\gamma_{n}\left(K\left[\boldsymbol{\theta}_{n}\right] Q\left[\boldsymbol{\theta}_{n}\right]\right)_{\boldsymbol{X}_{n}^{i}, x} & \ell=2 \\
\gamma_{n}\left(K\left[\boldsymbol{\theta}_{n}\right] Q\left[\boldsymbol{\theta}_{n}\right]\right)_{\boldsymbol{X}_{n}^{i}, x}-\gamma_{n+1}\left(K\left[\boldsymbol{\theta}_{n+1}\right] Q\left[\boldsymbol{\theta}_{n+1}\right]\right)_{\boldsymbol{X}_{n+1}^{i}, x} & \ell=3 \\
\gamma_{n+1}\left(K\left[\boldsymbol{\theta}_{n+1}\right] Q\left[\boldsymbol{\theta}_{n+1}\right]\right)_{\boldsymbol{X}_{n+1}^{i}, x}-\gamma_{n+1}\left(K\left[\boldsymbol{\theta}_{n}\right] Q\left[\boldsymbol{\theta}_{n}\right]\right)_{\boldsymbol{X}_{n+1}^{i}, x} & \ell=4
\end{array}\right.
$$

where $Q(\cdot)$ denotes the solution to the Poisson equation in (1.16).

For $1 \leq \ell \leq 4$, let

$$
\delta_{n+1}^{\ell} \doteq \frac{1}{a(n+1)} \sum_{i=1}^{a(n+1)} \delta_{n+1}^{\ell, i},
$$

and observe that with $\boldsymbol{\epsilon}_{n+1}$ as in (1.9),

$$
\gamma_{n+1} \boldsymbol{\epsilon}_{n+1}=\sum_{\ell=1}^{4} \delta_{n+1}^{\ell} .
$$

We will now establish several bounds on the error terms. The following lemma provides a bound for the martingale noise term, namely the term corresponding to $\ell=1$. Recall the function $m(\cdot)$ defined in $(2.6)$.

Lemma 5.2. For each $T \in(0, \infty)$,

$$
\lim _{t \rightarrow \infty} \sup _{0 \leq u \leq T}\left\|\sum_{j=m(t)}^{m(t+u)-1} \delta_{j+1}^{1}\right\|=0 .
$$

Proof. Note that $\left\{\delta_{j}^{1}\right\}_{j=1}^{\infty}$ is adapted to $\left\{\mathcal{F}_{j}\right\}_{j=1}^{\infty}$, and $\mathbf{E}\left[\delta_{j+1}^{1} \mid \mathcal{F}_{j}\right]=0$, where $\mathcal{F}_{j}$ is as introduced above (1.8). Thus, $\left\{\delta_{j}^{1}\right\}_{j=1}^{\infty}$ is a martingale difference sequence. Furthermore, there is some $\kappa_{1} \in(0, \infty)$ such that $\left\|\delta_{j+1}^{1}\right\| \leq$ $\kappa_{1} \gamma_{j+1}$ for each $j \in \mathbb{N}_{0}$. The result now follows by standard martingale estimates (see e.g. the proof of Prop. 4.4 in [4]). 
The next result provides bounds for the remaining error terms.

Lemma 5.3. For $\ell=2,3,4$ and $T>0$, we have that

$$
\lim _{t \rightarrow \infty} \sup _{0 \leq u \leq T}\left\|\sum_{j=m(t)}^{m(t+u)-1} \delta_{j+1}^{\ell}\right\|=0
$$

Proof. The proofs for the cases when $\ell=2$ and $\ell=4$ are similar to the proofs of Lemma 2.5 and Lemma 2.7, respectively, and are omitted. We now consider the case when $\ell=3$. Recall the sequence $\left\{b(n), n \in \mathbb{N}_{0}\right\}$ defined in (1.7). Note that for an array $\left\{\alpha_{j}^{i}, i, j \in \mathbb{N}\right\}$, we have, for $n \in \mathbb{N}$,

$$
\begin{gathered}
\sum_{j=0}^{n-1} \sum_{i=1}^{a(j+1)} \alpha_{j+1}^{i}=\sum_{j=0}^{n-1} \sum_{i=1}^{a(n)} \alpha_{j+1}^{i} \mathbb{1}_{\{1 \leq i \leq a(j+1)\}}=\sum_{i=1}^{a(n)} \sum_{j=0}^{n-1} \alpha_{j+1}^{i} \mathbb{1}_{\{1 \leq i \leq a(j+1)\}} \\
=\sum_{j=0}^{n-1} \alpha_{j+1}^{1}+\sum_{i=2}^{a(n)} \sum_{j=0}^{n-1} \alpha_{j+1}^{i} \mathbb{1}_{\{1 \leq i \leq a(j+1)\}}=\sum_{j=0}^{n-1} \alpha_{j+1}^{1}+\sum_{i=2}^{a(n)} \sum_{j=0}^{n-1} \alpha_{j+1}^{i} \mathbb{1}_{\{0 \leq b(i) \leq j+1\}} \\
=\sum_{j=0}^{n-1} \alpha_{j+1}^{1}+\sum_{i=2}^{a(n)} \sum_{j=b(i)-1}^{n-1} \alpha_{j+1}^{i} .
\end{gathered}
$$

Let, for $j \in \mathbb{N}_{0}, \beta_{j}^{i} \doteq \gamma_{j}\left(K\left[\theta_{j}\right] Q\left[\theta_{j}\right]\right)_{\boldsymbol{X}_{j}^{i}}$, so that,

$$
\delta_{j+1}^{3}=\frac{1}{a(j+1)} \sum_{i=1}^{a(j+1)} \delta_{j+1}^{3, i}=\sum_{i=1}^{a(j+1)} \frac{\beta_{j}^{i}-\beta_{j+1}^{i}}{a(j+1)} .
$$

Then, using (5.5),

$$
\begin{aligned}
\sum_{j=0}^{n-1} \delta_{j+1}^{3} & =\sum_{j=0}^{n-1} \frac{\beta_{j}^{1}-\beta_{j+1}^{1}}{a(j+1)}+\sum_{i=2}^{a(n)} \sum_{j=b(i)-1}^{n-1} \frac{\beta_{j}^{i}-\beta_{j+1}^{i}}{a(j+1)} \\
& =\sum_{j=0}^{n-1} \frac{\beta_{j}^{1}-\beta_{j+1}^{1}}{a(j+1)}+\sum_{i=2}^{a(n)}\left[\frac{\beta_{b(i)-1}^{i}}{a(b(i))}-\frac{\beta_{n}^{i}}{a(n)}+\sum_{j=b(i)}^{n-1}\left(\frac{\beta_{j}^{i}}{a(j+1)}-\frac{\beta_{j}^{i}}{a(j)}\right)\right]
\end{aligned}
$$

For $t, u>0$, let

$$
n \doteq n(t, u) \doteq m(t+u), \quad m \doteq m(t)
$$


where $m(t)$ is given by (2.6). Observe that

$$
\begin{aligned}
\sum_{j=m}^{n-1} \delta_{j+1}^{3}= & \sum_{j=0}^{n-1} \delta_{j+1}^{3}-\sum_{j=0}^{m-1} \delta_{j+1}^{3} \\
= & \sum_{j=m}^{n-1} \frac{\beta_{j}^{1}-\beta_{j+1}^{1}}{a(j+1)}+\sum_{i=a(m)+1}^{a(n)} \frac{\beta_{b(i)-1}^{i}}{a(b(i))}+\sum_{i=2}^{a(m)} \frac{\beta_{m}^{i}}{a(m)}-\sum_{i=2}^{a(n)} \frac{\beta_{n}^{i}}{a(n)} \\
& +\sum_{i=2}^{a(n)} \sum_{j=b(i)}^{n-1}\left(\frac{\beta_{j}^{i}}{a(j+1)}-\frac{\beta_{j}^{i}}{a(j)}\right)-\sum_{i=2}^{a(m)} \sum_{j=b(i)}^{m-1}\left(\frac{\beta_{j}^{i}}{a(j+1)}-\frac{\beta_{j}^{i}}{a(j)}\right) .
\end{aligned}
$$

The last expression can be rewritten as

$$
\begin{aligned}
& \sum_{j=m}^{n-1} \frac{\beta_{j}^{1}-\beta_{j+1}^{1}}{a(j+1)}+\sum_{i=a(m)+1}^{a(n)} \frac{\beta_{b(i)-1}^{i}}{a(b(i))}+\sum_{i=2}^{a(m)} \frac{\beta_{m}^{i}}{a(m)}-\sum_{i=2}^{a(n)} \frac{\beta_{n}^{i}}{a(n)} \\
& \quad+\sum_{i=2}^{a(m)} \sum_{j=m}^{n-1}\left(\frac{\beta_{j}^{i}}{a(j+1)}-\frac{\beta_{j}^{i}}{a(j)}\right)+\sum_{i=a(m)+1}^{a(n)} \sum_{j=b(i)}^{n-1}\left(\frac{\beta_{j}^{i}}{a(j+1)}-\frac{\beta_{j}^{i}}{a(j)}\right) .
\end{aligned}
$$

Define

$$
\begin{aligned}
\eta_{0}(n, m) \doteq \sum_{j=m}^{n-1} \frac{\beta_{j}^{1}-\beta_{j+1}^{1}}{a(j+1)}, \eta_{1}(n, m) & \doteq \sum_{i=a(m)+1}^{a(n)} \frac{\beta_{b(i)-1}^{i}}{a(b(i))}+\sum_{i=2}^{a(m)} \frac{\beta_{m}^{i}}{a(m)}-\sum_{i=2}^{a(n)} \frac{\beta_{n}^{i}}{a(n)}, \\
\eta_{2}(n, m) \doteq \sum_{i=2}^{a(m)} \sum_{j=m}^{n-1}\left(\frac{\beta_{j}^{i}}{a(j+1)}-\frac{\beta_{j}^{i}}{a(j)}\right), \eta_{3}(n, m) & \doteq \sum_{i=a(m)+1}^{a(n)} \sum_{j=b(i)}^{n-1}\left(\frac{\beta_{j}^{i}}{a(j+1)}-\frac{\beta_{j}^{i}}{a(j)}\right) .
\end{aligned}
$$

Then

$$
\sum_{j=m}^{n-1} \delta_{j+1}^{3}=\sum_{\ell=0}^{3} \eta_{\ell}(n, m)
$$

We begin by considering $\eta_{3}(n, m)$. Let $\kappa_{1} \doteq \sup _{\theta \in \mathcal{P}\left(\Delta^{o}\right)}\left\|K[\theta] Q[\theta]_{x},\right\|$, so that $\left\|\beta_{j}^{i}\right\| \leq \kappa_{1} \gamma_{j}$. Note that there is some $\kappa_{2} \in(0, \infty)$ such that $\frac{\gamma_{j}}{a(j)}-\frac{\gamma_{j}}{a(j+1)} \leq \kappa_{2} \frac{\gamma_{j}^{x}}{a(j)^{2}}$. Using the last two estimates, the form of $\gamma_{j}$, and the fact that $a(j) \sim j^{\zeta}$, we can find some $\kappa_{3} \in(0, \infty)$ such that

$$
\left\|\frac{\beta_{j}^{i}}{a(j+1)}-\frac{\beta_{j}^{i}}{a(j)}\right\| \leq \frac{\kappa_{3}}{j^{1+2 \zeta}} .
$$

Note that if $1 \leq i \leq a(n)$, then $b(i) \leq n$, so $b(i)^{-2 \zeta} \geq n^{-2 \zeta}$. It follows that there is some $\kappa_{4} \in(0, \infty)$ such that

$$
\left\|\eta_{3}(n, m)\right\| \leq \kappa_{3} \sum_{i=a(m)+1}^{a(n)} \sum_{j=b(i)}^{n} \frac{1}{j^{1+2 \zeta}} \leq \kappa_{4} \sum_{i=a(m)+1}^{a(n)}\left(\frac{1}{(b(i)-1)^{2 \zeta}}-\frac{1}{n^{2 \zeta}}\right) \leq \kappa_{4} \frac{a(n)}{b(a(m))^{2 \zeta}} .
$$


Note that there are some $c_{1}, c_{2} \in(0, \infty)$ and some $t_{1} \in(0, \infty)$ such that if $t \geq t_{1}$, then $c_{1} m(t)^{\zeta} \leq a(m(t)) \leq$ $c_{2} m(t)^{\zeta}$. From the definition of $a(\cdot)$ and $b(\cdot)$, we see that $b(a(m)) \sim m$. Fix $\epsilon \in(0,1)$. Then, there is a $t_{2} \in\left(t_{1}, \infty\right)$ such that if $t \geq t_{2}$, then $b(a(m(t))) \geq(1-\epsilon) m(t)$. It follows that if $t \geq t_{2}$, then

$$
\frac{a(n(t, u))}{b\left(a(m(t))^{2 \zeta}\right.} \leq \frac{c_{2} n(t, u)^{\zeta}}{(1-\epsilon)^{2 \zeta} m(t)^{2 \zeta}}
$$

Recall that $\tau_{k}=\sum_{j=1}^{k} \gamma_{j} \sim \gamma_{*} \log (k)$. From this and the definition of $m(\cdot)$ it follows that, with $\alpha=1 / \gamma_{*}$, for some $t_{3} \in\left(t_{2}, \infty\right)$ and $c_{3}, c_{4} \in(0, \infty)$, and all $t \geq t_{3}$,

$$
c_{3} \exp (4 \alpha t / 5) \leq m(t) \leq c_{4} \exp (3 \alpha t / 2)
$$

Combining the previous display and (5.10), we see that if $t \geq t_{3}$, then

$$
\frac{a(n(t, u))}{b\left(a(m(t))^{2 \zeta}\right.} \leq \frac{c_{2} n(t, u)^{\zeta}}{(1-\epsilon)^{2 \zeta} m(t)^{2 \zeta}} \leq \frac{c_{2} c_{4}^{\zeta} e^{3 \zeta \alpha(t+u) / 2}}{c_{3}^{2 \zeta}(1-\epsilon)^{2 \zeta} e^{8 \zeta \alpha t / 5}} \leq \frac{c_{2} c_{4}^{\zeta} e^{2 \zeta \alpha u}}{c_{3}^{2 \zeta}(1-\epsilon)^{2 \zeta} e^{\zeta \alpha t / 10}}
$$

Let $\kappa_{6} \doteq \kappa_{6}(T) \doteq \frac{c_{2} c_{4}^{\zeta} e^{2 \zeta \alpha T}}{c_{3}^{\zeta \zeta}(1-\epsilon)^{2 \zeta}}$. Then combining (5.9), (5.10), and (5.11), we see that if $t \geq t_{3}$, then $\sup _{0 \leq u \leq T}\left\|\eta_{3}(n(t, u), m(t))\right\| \leq \frac{\kappa_{6}}{e^{\zeta \alpha t / 10}}$, and so, as $t \rightarrow \infty$,

$$
\sup _{0 \leq u \leq T}\left\|\eta_{3}(n(t, u), m(t))\right\| \rightarrow 0
$$

We now consider $\eta_{2}(n, m)$. From (5.8), there is some $\kappa_{7} \in(0, \infty)$ such that

$$
\left\|\eta_{2}(n, m)\right\| \leq \kappa_{3} \sum_{i=2}^{a(m)} \sum_{j=m}^{n-1} \frac{1}{j^{1+2 \zeta}} \leq \kappa_{7} \sum_{i=1}^{a(m)} \frac{1}{m^{2 \zeta}}=\kappa_{7} a(m) \frac{1}{m^{2 \zeta}}
$$

which shows that as $t \rightarrow \infty$,

$$
\sup _{0 \leq u \leq T}\left\|\eta_{2}(n(t, u), m(t))\right\| \rightarrow 0
$$

We now consider $\eta_{1}(n, m)$. We can find some $\kappa_{8} \in(0, \infty)$ such that

$$
\left\|\eta_{1}(n, m)\right\| \leq \kappa_{8}\left[\sum_{i=a(m)+1}^{a(n)} \frac{\gamma_{b(i)-1}}{a(b(i))}+\sum_{i=1}^{a(n)} \frac{\gamma_{n}}{a(n)}+\sum_{i=1}^{a(m)} \frac{\gamma_{m}}{a(m)}\right]=\kappa_{8}\left[\gamma_{m}+\gamma_{n}+\sum_{i=a(m)+1}^{a(n)} \frac{\gamma_{b(i)-1}}{a(b(i))}\right] .
$$

Note that $a(b(i))=i$, so there is some $\kappa_{9} \in(0, \infty)$ such that

$$
\sum_{i=a(m)+1}^{a(n)} \frac{\gamma_{b(i)-1}}{a(b(i))} \leq \kappa_{9} \sum_{i=a(m)+1}^{a(n)} \frac{1}{i b(i)}
$$


Additionally, $b(i) \sim i^{1 / \zeta}$, so we can find some $t_{4} \in(0, \infty)$ such that if $t \geq t_{4}$ and $i \geq a(m(t))$, then $\frac{1}{b(i)} \leq$ $(1+\epsilon) \frac{1}{i^{1 / \zeta}}$. It follows that there is some $\kappa_{10} \in(0, \infty)$ such that if $t \geq t_{4}$,

$$
\sum_{i=a(m)+1}^{a(n)} \frac{\gamma_{b(i)-1}}{a(b(i))} \leq \kappa_{9}(1+\epsilon) \sum_{i=a(m)}^{a(n)} \frac{1}{i^{1+1 / \zeta}} \leq \kappa_{10} \frac{1}{a(m)^{1 / \zeta}}
$$

Combining (5.15) and (5.16), we see that

$$
\sup _{0 \leq u \leq T}\left\|\eta_{1}(n(t, u), m(t))\right\| \rightarrow 0
$$

as $t \rightarrow \infty$. Finally, consider $\eta_{0}(n, m)$. We have, for some $\kappa_{11} \in(0, \infty)$, that $\left\|\eta_{0}(n, m)\right\| \leq \kappa_{11} \sum_{j=m}^{n} \frac{1}{j^{1+\zeta}}$, so it follows that as $t \rightarrow \infty$.

$$
\sup _{0 \leq u \leq T}\left\|\eta_{0}(n(t, u), m(t))\right\| \rightarrow 0
$$

Combining (5.7), (5.12), (5.14), (5.17), and (5.18) we see that the convergence in (5.4) holds with $\ell=3$. The result follows.

Define the continuous-time process $\{\bar{\epsilon}(t), t \geq 0\}$ by

$$
\bar{\epsilon}\left(\tau_{n}+t\right) \doteq \boldsymbol{\epsilon}_{n+1}, \quad t \in\left[0, \gamma_{k+1}\right), n \in \mathbb{N}_{0},
$$

and define

$$
\Delta(t, T) \doteq \sup _{0 \leq u \leq T}\left\|\int_{t}^{t+u} \bar{\epsilon}(s) \mathrm{d} s\right\|, t, T \geq 0 .
$$

We now complete the proof of Theorem 1.4.

Proof of Theorem 1.4: Fix $T \in(0, \infty)$. Then, for some $\kappa_{1} \in(0, \infty)$, and all $t>0$.

$$
\begin{aligned}
\Delta(t, T) & \leq \sup _{0 \leq u \leq T}\left\|\int_{\tau_{m(t)}}^{\tau_{m(t+u)}} \bar{\epsilon}(s) \mathrm{d} s\right\|+\sup _{0 \leq u \leq T}\left\|\int_{\tau_{m(t)}}^{t} \bar{\epsilon}(s) \mathrm{d} s\right\|+\sup _{0 \leq u \leq T}\left\|\int_{\tau_{m(t+u)}}^{t+u} \bar{\epsilon}(s) \mathrm{d} s\right\| \\
& \leq \sum_{k=1}^{4} \sup _{0 \leq u \leq T}\left\|\sum_{j=m(t)}^{m(t+u)-1} \delta_{j+1}^{k}\right\|+2 \kappa_{1} \gamma_{m(t)} .
\end{aligned}
$$

From Lemma 5.2 and Lemma 5.3 we now have that $\lim _{t \rightarrow \infty} \Delta(t, T)=0$. From Proposition 4.1 of [3] it follows that $\{\hat{\boldsymbol{\theta}}(t)\}$ is an asymptotic pseudo-trajectory. The result now follows exactly as in the proof of $[6]^{*}$ Theorem 1.2 .

\section{Central limit theorem for Algorithm II}

In this section we provide the proof of Theorem 1.5. In Section 6.1, we characterize the covariance structure of the error sequence $\left\{\boldsymbol{e}_{n}\right\}$. In Section 6.2 we present some results for the linearized evolution sequence $\left\{\boldsymbol{\mu}_{n}\right\}$, and in Section 6.3 we characterize the asymptotic behavior of the discrepancy sequence $\left\{\boldsymbol{\rho}_{n}\right\}$. The proof of Theorem 1.5 is completed in Section 6.4.

We begin by studying the covariance structure of the error terms. 


\subsection{Covariance structure of the error terms}

Recall the collection of matrices $\left\{F_{\theta}(z): \theta \in \mathcal{P}\left(\Delta^{o}\right), z \in \Delta^{o}\right\}$ defined by (3.1) and let $U_{*}$ be the $d \times d$ matrix introduced in (3.2). The following result gives an expression for the conditional covariance matrix of $\left\{\boldsymbol{e}_{n+1}\right\}$ introduced in (1.22). The proof is similar to the proof of Proposition 3.1.

Proposition 6.1. For each $n \in \mathbb{N}_{0}$ and $x, y \in \Delta^{o}$,

$$
\mathbf{E}\left[\boldsymbol{e}_{n+1}(x) \boldsymbol{e}_{n+1}(y) \mid \mathcal{F}_{n}\right]=\frac{1}{a(n+1)}\left(\left(U_{*}\right)_{x, y}+\left(D_{n}^{(1)}\right)_{x, y}+\left(D_{n}^{(2)}\right)_{x, y}\right),
$$

where $D_{n}^{(1)}$ and $D_{n}^{(2)}$ are $d \times d$ random matrices satisfying the following:

(i) for some $C_{1} \in(0, \infty)$ and all $n \in \mathbb{N},\left\|D_{n}^{(1)}\right\| \leq C_{1}\left\|\boldsymbol{\theta}_{n}-\theta_{*}\right\|$.

(ii) for some $C_{2}, \beta \in(0, \infty)$ and for all $n \in \mathbb{N}$,

$$
\gamma_{n+1} \mathbf{E}\left\|\sum_{m=1}^{n} D_{m-1}^{(2)}\right\| \leq C n^{-\beta}
$$

Proof. By a similar argument as in the proof of Proposition 3.1, we have that

$$
\mathbf{E}\left[\boldsymbol{e}_{n+1}(x) \boldsymbol{e}_{n+1}(y) \mid \mathcal{F}_{n}\right]=a(n+1)^{-2} \sum_{i=1}^{a(n+1)} F_{\boldsymbol{\theta}_{n}}\left(\boldsymbol{X}_{n}^{i}\right)_{x, y}
$$

We can write

$$
F_{\boldsymbol{\theta}_{n}}\left(\boldsymbol{X}_{n}^{i}\right)_{x, y}=\left(U_{*}\right)_{x, y}+\left(D_{n}^{(1), i}\right)_{x, y}+\left(D_{n}^{(2), i}\right)_{x, y}
$$

where

$$
D_{n}^{(1), i} \doteq \sum_{w \in \Delta^{o}}\left(F_{\boldsymbol{\theta}_{n}}(w) \pi\left(\boldsymbol{\theta}_{n}\right)_{w}-F_{\theta_{*}}(w)\left(\theta_{*}\right)_{w}\right) \text { and } D_{n}^{(2), i} \doteq F_{\boldsymbol{\theta}_{n}}\left(\boldsymbol{X}_{n}^{i}\right)-\sum_{w \in \Delta^{o}} F_{\boldsymbol{\theta}_{n}}(w) \pi\left(\boldsymbol{\theta}_{n}\right)_{w}
$$

The identity in (6.1) is obtained by defining

$$
D_{n}^{(1)} \doteq \frac{1}{a(n+1)} \sum_{i=1}^{a(n+1)} D_{n}^{(1), i}=\sum_{w \in \Delta^{o}}\left(F_{\boldsymbol{\theta}_{n}}(w) \pi\left(\boldsymbol{\theta}_{n}\right)_{w}-F_{\theta_{*}}(w)\left(\theta_{*}\right)_{w}\right),
$$

and

$$
D_{n}^{(2)} \doteq \frac{1}{a(n+1)} \sum_{i=1}^{a(n+1)} D_{n}^{(2), i}
$$

and using the identity in (6.2). The proof of (i) is similar to the proof of part (i) of Proposition 3.1 and is omitted. We now show that (ii) holds as well. 
Proof of (ii): As in the proof of part (ii) of Proposition 3.1, it suffices to show that there is some $C_{2}, \beta>0$ such that for each $(u, v) \in \Delta^{o} \times \Delta^{o}$ and all $n \in \mathbb{N}$,

$$
\gamma_{n+1} \mathbf{E}\left\|\sum_{m=1}^{n}\left(D_{m}^{(2)}\right)_{u, v}\right\| \leq C_{2}(n+1)^{-\beta}
$$

Fix $(u, v) \in \Delta^{o} \times \Delta^{o}$ and, once more abusing notation, denote $\left(D_{m}^{(2)}\right)_{u, v}$ as $D_{m}^{(2)}$. Using the Poisson equation (1.16) we have, as in the proof of Proposition 3.1, with

$$
D_{n}^{(2, a), i} \doteq U_{\boldsymbol{\theta}_{n}}\left(\boldsymbol{X}_{n+1}^{i}\right)-\left(K\left[\boldsymbol{\theta}_{n}\right] U_{\boldsymbol{\theta}_{n}}\right)\left(\boldsymbol{X}_{n}^{i}\right), \quad D_{n}^{(2, b), i} \doteq U_{\boldsymbol{\theta}_{n}}\left(\boldsymbol{X}_{n}^{i}\right)-U_{\boldsymbol{\theta}_{n}}\left(\boldsymbol{X}_{n+1}^{i}\right),
$$

that $D_{n}^{(2), i}=D_{n}^{(2, a), i}+D_{n}^{(2, b), i}$. Now, let

$$
D_{n}^{(2, a)} \doteq \frac{1}{a(n+1)} \sum_{i=1}^{a(n+1)} D_{n}^{(2, a), i}, \quad D_{n}^{(2, b)} \doteq \frac{1}{a(n+1)} \sum_{i=1}^{a(n+1)} D_{n}^{(2, b), i}
$$

so that $D_{n}^{(2)}=D_{n}^{(2, a)}+D_{n}^{(2, b)}$. Note that with $\mathcal{G}_{n} \doteq \mathcal{F}_{n+1},\left\{D_{n}^{(2, a)}\right\}_{n=1}^{\infty}$ is a $\left\{\mathcal{G}_{n}\right\}_{n=1}^{\infty}$-martingale increment sequence. Consequently, we can apply Burkholder's inequality and use a conditioning argument to show that, for some $\kappa_{1} \in(0, \infty)$, and all $n \in \mathbb{N}$,

$$
\gamma_{n+1} \mathbf{E}\left|\sum_{m=1}^{n} D_{m-1}^{(2, a)}\right| \leq \kappa_{1} \gamma_{n+1}\left(\sum_{m=1}^{n} \frac{1}{a(m)}\right)^{1 / 2} .
$$

We now consider $\left\{D_{n}^{(2, b)}\right\}$. Observe that

$$
\begin{aligned}
\left|\sum_{m=1}^{n} D_{m-1}^{(2, b)}\right| \leq & \left|\frac{1}{a(1)} \sum_{i=1}^{a(1)} U_{\theta_{1}}\left(\boldsymbol{X}_{1}^{i}\right)-\frac{1}{a(n)} \sum_{i=1}^{a(n)} U_{\boldsymbol{\theta}_{n}}\left(\boldsymbol{X}_{n+1}^{i}\right)\right| \\
& +\mid \sum_{m=2}^{n}\left[\frac{1}{a(m)} \sum_{i=1}^{a(m)} U_{\theta_{m}}\left(\boldsymbol{X}_{m}^{i}\right)-\frac{1}{a(m-1)} \sum_{i=1}^{a(m-1)} U_{\theta_{m-1}}\left(\boldsymbol{X}_{m}^{i}\right)\right],
\end{aligned}
$$

and

$$
\begin{aligned}
& \left|\sum_{m=2}^{n}\left[\frac{1}{a(m)} \sum_{i=1}^{a(m)} U_{\theta_{m}}\left(\boldsymbol{X}_{m}^{i}\right)-\frac{1}{a(m-1)} \sum_{i=1}^{a(m-1)} U_{\theta_{m-1}}\left(\boldsymbol{X}_{m}^{i}\right)\right]\right| \\
& \leq\left|\sum_{m=2}^{n} \frac{1}{a(m)} \sum_{i=a(m-1)+1}^{a(m)} U_{\theta_{m}}\left(\boldsymbol{X}_{m}^{i}\right)\right|+\left|\sum_{m=2}^{n} \sum_{i=1}^{a(m-1)}\left[\frac{1}{a(m)} U_{\theta_{m}}\left(\boldsymbol{X}_{m}^{i}\right)-\frac{1}{a(m-1)} U_{\theta_{m-1}}\left(\boldsymbol{X}_{m}^{i}\right)\right]\right| .
\end{aligned}
$$

Letting $\kappa_{2} \doteq \sup _{\theta \in \mathcal{P}\left(\Delta^{\circ}\right), z \in \Delta^{\circ}}\left|U_{\theta}(z)\right|<\infty$, and noting that $0 \leq a(m)-a(m-1) \leq 1$ for all $m \in \mathbb{N}$, we see that

$$
\left|\sum_{m=2}^{n} \frac{1}{a(m)} \sum_{i=a(m-1)+1}^{a(m)} U_{\theta_{m}}\left(\boldsymbol{X}_{m}^{i}\right)\right| \leq \kappa_{2} \sum_{m=2}^{n} \frac{1}{a(m)} .
$$


Since the maps $\theta \mapsto K(\theta)$ and $\theta \mapsto Q(\theta)$ are bounded Lipschitz maps, there is a $\kappa_{3} \in(0, \infty)$ such that for all $x \in \Delta^{o}$ and $\theta, \theta^{\prime} \in \mathcal{P}\left(\Delta^{o}\right),\left|U_{\theta}(x)-U_{\theta^{\prime}}(x)\right| \leq \kappa_{3}\left\|\theta-\theta^{\prime}\right\|$, so there is some $\kappa_{4} \in(0, \infty)$ such that

$$
\begin{aligned}
& \left|\sum_{m=2}^{n} \sum_{i=1}^{a(m-1)}\left[\frac{1}{a(m)} U_{\theta_{m}}\left(\boldsymbol{X}_{m}^{i}\right)-\frac{1}{a(m-1)} U_{\theta_{m-1}}\left(\boldsymbol{X}_{m}^{i}\right)\right]\right| \\
& \leq \sum_{m=2}^{n} \sum_{i=1}^{a(m-1)}\left[\left|\frac{1}{a(m)} U_{\theta_{m}}\left(\boldsymbol{X}_{m}^{i}\right)-\frac{1}{a(m-1)} U_{\theta_{m}}\left(\boldsymbol{X}_{m}^{i}\right)\right|+\left|\frac{1}{a(m-1)}\left(U_{\theta_{m}}\left(\boldsymbol{X}_{m}^{i}\right)-U_{\theta_{m-1}}\left(\boldsymbol{X}_{m}^{i}\right)\right)\right|\right] \\
& \leq \sum_{m=2}^{n} \sum_{i=1}^{a(m-1)}\left[\kappa_{2} \frac{1}{a(m-1) a(m)}+2 \kappa_{3} \gamma_{m} \frac{1}{a(m-1)}\right] \leq \kappa_{4} \sum_{m=1}^{n}\left[\frac{1}{a(m)}+\gamma_{m}\right] .
\end{aligned}
$$

Additionally,

$$
\left|\frac{1}{a(1)} \sum_{i=1}^{a(1)} U_{\theta_{1}}\left(\boldsymbol{X}_{1}^{i}\right)-\frac{1}{a(n)} \sum_{i=1}^{a(n)} U_{\boldsymbol{\theta}_{n}}\left(\boldsymbol{X}_{n+1}^{i}\right)\right| \leq 2 \kappa_{2} .
$$

Combining (6.4),(6.5), (6.6), (6.7), and (6.8) we see that there is some $\kappa_{5} \in(0, \infty)$ such that

$$
\gamma_{n+1}\left\|\sum_{m=1}^{n} D_{m-1}^{(2, b)}\right\| \leq \kappa_{5} \gamma_{n+1}\left(1+\sum_{m=1}^{n}\left[\frac{1}{a(m)}+\gamma_{m}\right]\right) .
$$

The result follows on combining (6.3) and (6.9).

The next result provides a useful bound for the moments of the error sequence $\left\{\boldsymbol{e}_{n}\right\}$. The proof is similar to the proof of Proposition 3.2 and is omitted.

Proposition 6.2. There is some $C>0$ such that for all $n \in \mathbb{N}, \mathbf{E}\left\|\boldsymbol{e}_{n}\right\|^{4} \leq C / a(n)^{2}$.

\subsection{The linearized evolution sequence}

The goal of this section is to study the linearized evolution sequence given in (1.25). The following lemma says that $\boldsymbol{\mu}_{n}$ given by the linearized evolution in (1.25) converges a.s. to 0 . The proof is similar to the proof of Proposition 5.1 in [21] and Proposition 4.1, and is therefore omitted.

Lemma 6.3. As $n \rightarrow \infty$ we have $\boldsymbol{\mu}_{n} \rightarrow 0$ a.s.

The next result is used in the proof of Proposition 6.6. It provides a useful bound on the moments of the linearized evolution sequence. Recall the quantity $\sigma_{n}=\sqrt{a(n) / \gamma_{n}}$ defined in (1.11).

Proposition 6.4. Suppose that $\gamma_{*}>L^{-1}$. Then there is some $C \in(0, \infty)$ such that for all $n \in \mathbb{N}_{0}, \mathbf{E}\left\|\boldsymbol{\mu}_{n+1}\right\|^{2} \leq$ $C / \sigma_{n+1}^{2}$.

Proof. Recall the collection of matrices $\left\{\psi_{*}(n, k), n \in \mathbb{N}, k \leq n+1\right\}$ defined in (4.1). A simple recursive argument shows that

$$
\boldsymbol{\mu}_{n+1}=\sum_{k=1}^{n+1} \gamma_{k} \psi_{*}(n+1, k+1) \boldsymbol{e}_{k}
$$


Proposition 6.2 ensures that there is some $\kappa_{1} \in(0, \infty)$ such that $\mathbf{E}\left\|\boldsymbol{e}_{k}\right\|^{2} \leq \kappa_{1} / a(k)$ for all $k \in \mathbb{N}$. Fix $L^{\prime} \in(0, L)$ such that $L^{\prime} \gamma_{*}>1$, and use (4.3) and (6.10) to find some $\kappa_{2}\left(L^{\prime}\right) \in(0, \infty)$ such that

$$
\mathbf{E}\left\|\boldsymbol{\mu}_{n+1}\right\|^{2} \leq \kappa_{2}\left(L^{\prime}\right) \sum_{k=1}^{n+1} \gamma_{k}^{2} \exp \left(-2 L^{\prime} \sum_{j=k+1}^{n+1} \gamma_{j}\right) \frac{1}{a(k)} .
$$

Note that there is some $\kappa_{3} \in(0, \infty)$ such that

$$
\sigma_{n}^{2} \leq \kappa_{3} n^{1+\zeta}, \quad n \in \mathbb{N}
$$

so there is some $\kappa_{4} \in(0, \infty)$ such that

$$
\sigma_{n+1}^{2} \mathbf{E}\left\|\boldsymbol{\mu}_{n+1}\right\|^{2} \leq \kappa_{4} n^{1+\zeta} \sum_{k=1}^{n+1} \gamma_{k}^{2+\zeta} \exp \left(-2 L^{\prime} \sum_{j=k+1}^{n+1} \gamma_{j}\right) .
$$

The right side is bounded since $\gamma_{*} L^{\prime}>1$. The result follows.

\subsection{Analysis of the discrepancy sequence}

The goal of this section is to show that the discrepancy sequence $\left\{\boldsymbol{\rho}_{n}\right\}$ converges to 0 in probability under the central limit scaling. As in Section 4.2, for each $n \in \mathbb{N}$, we let $R_{\bullet}^{(n)}$ denote the tensor $R_{i}^{(n)} \doteq \mathbf{R}_{i}^{(n)}\left(\boldsymbol{\theta}_{n}\right)$, $1 \leq i \leq d$, where $\mathbf{R}_{i}^{(n)}(\theta)$, for $\theta \in \mathcal{P}\left(\Delta^{o}\right)$, is defined as in (4.4). Note that this tensor satisfies

$$
h\left(\boldsymbol{\theta}_{n}\right)=\nabla h\left(\theta_{*}\right)\left(\boldsymbol{\theta}_{n}-\theta_{*}\right)+\left(\boldsymbol{\theta}_{n}-\theta_{*}\right)^{T} R_{\bullet}^{(n)}\left(\boldsymbol{\theta}_{n}-\theta_{*}\right) .
$$

For each $1 \leq k \leq n$, define the matrix $\psi(n, k)$ by

$$
\psi(n, k) \doteq \prod_{j=k}^{n}\left(I+\gamma_{j}\left(\nabla h\left(\theta_{*}\right)+2 \boldsymbol{\mu}_{j-1}^{T} R_{\bullet}^{(j-1)}+\boldsymbol{\rho}_{j-1}^{T} R_{\bullet}^{(j-1)}\right)\right),
$$

and let $\psi(n, n+1) \doteq I$. The next proposition provides a useful bound on $\psi(n, k)$.

Proposition 6.5. For each $L^{\prime} \in(0, L)$, and a.e. $\omega$, there is a $C=C\left(L^{\prime}, \omega\right) \in(0, \infty)$ such that if $1 \leq k \leq n$, then

$$
\|\psi(n, k)\| \leq C \exp \left(-L^{\prime} \sum_{j=k}^{n} \gamma_{j}\right)
$$

Proof. Let $A \doteq \nabla h\left(\theta_{*}\right)$ and

$$
A_{n} \doteq \nabla h\left(\theta_{*}\right)+2 \boldsymbol{\mu}_{j-1}^{T} R_{\bullet}^{(j-1)}+\boldsymbol{\rho}_{j-1}^{T} R_{\bullet}^{(j-1)}=\nabla h\left(\theta_{*}\right)+\boldsymbol{\mu}_{j-1}^{T} R_{\bullet}^{(j-1)}+\left(\theta_{j-1}-\theta_{*}\right)^{T} R_{\bullet}^{(j-1)} .
$$

From Theorem 1.4 and Lemma 6.3, $\left\|A_{n}-A\right\| \rightarrow 0$ a.s. as $n \rightarrow \infty$. The result now follows from Lemma 5.8 of [21].

The next result will be used to show that $\left\{\boldsymbol{\rho}_{n}\right\}$ tends to 0 in probability under the central limit scaling. 
Proposition 6.6. Suppose $\gamma_{*}>L^{-1}$. For some $\kappa \in(0,1)$, we have, as $n \rightarrow \infty$,

$$
\sigma_{n}^{1+\kappa}\left(\boldsymbol{\rho}_{n}-\sum_{k=1}^{n} \gamma_{k} \psi(n, k+1) \boldsymbol{r}_{k}\right) \stackrel{\mathbf{P}}{\rightarrow} 0
$$

Proof. Fix $L^{\prime} \in\left(\gamma_{*}^{-1}, L\right)$ and $\kappa \in\left(0, \frac{1}{2} \wedge\left[2 L^{\prime} \gamma_{*}(1+\zeta)^{-1}-1\right]\right)$. Using (1.24), (1.25), (1.26), (6.12), and a recursive argument similar to the one used for obtaining (4.7), we have

$$
\boldsymbol{\rho}_{n}-\sum_{k=1}^{n} \gamma_{k} \psi(n, k+1) \boldsymbol{r}_{k}=\psi(n, 1) \boldsymbol{\rho}_{0}+\sum_{k=1}^{n} \gamma_{k} \psi(n, k+1)\left(\boldsymbol{\mu}_{k-1}^{T} R_{\bullet}^{(k-1)} \boldsymbol{\mu}_{k-1}\right) \text {. }
$$

We begin by showing that as $n \rightarrow \infty$,

$$
\sigma_{n}^{1+\kappa} \psi(n, 1) \boldsymbol{\rho}_{0} \stackrel{\mathbf{P}}{\rightarrow} 0
$$

Since $\left\|\boldsymbol{\rho}_{0}\right\|$ is bounded, it suffices to show that $\sigma_{n}^{1+\kappa} \psi(n, 1)$ tends to 0 in probability. Using Proposition 6.5 , for a.e. $\omega$, there is some $\kappa_{1}(\omega) \in(0, \infty)$ such that

$$
\|\psi(n, 1)\| \leq \kappa_{1} \exp \left(-L^{\prime} \sum_{j=1}^{n} \gamma_{j}\right)
$$

Additionally, we can find some $\kappa_{2} \in(0, \infty)$ such that

$$
\exp \left(-L^{\prime} \sum_{j=1}^{n} \gamma_{j}\right) \leq \kappa_{2} n^{-L^{\prime} \gamma_{*}}, \quad \sigma_{n}^{1+\kappa} \leq \kappa_{2} n^{\frac{1}{2}(1+\kappa)(1+\zeta)} .
$$

Combining (6.16) and (6.17), we obtain, for a.e. $\omega$, some $\kappa_{3}(\omega) \in(0, \infty)$ such that

$$
\sigma_{n}^{1+\kappa}\|\psi(n, 1)\| \leq \kappa_{3} n^{\frac{1}{2}(1+\kappa)(1+\zeta)} n^{-L^{\prime} \gamma_{*}} .
$$

Since $\kappa \in\left(0, \frac{1}{2} \wedge\left[2 L^{\prime} \gamma_{*}(1+\zeta)^{-1}-1\right]\right)$, we have that $\frac{1}{2}(1+\kappa)(1+\zeta)-L^{\prime} \gamma_{*}<0$, which ensures that the expression in (6.18) converges to 0 . Therefore, (6.15) holds. We now show that as $n \rightarrow \infty$,

$$
\sigma_{n}^{1+\kappa} \sum_{k=1}^{n} \gamma_{k} \psi(n, k+1)\left(\boldsymbol{\mu}_{k-1}^{T} R_{\bullet}^{(k-1)} \boldsymbol{\mu}_{k-1}\right) \stackrel{\mathbf{P}}{\rightarrow} 0
$$

Note that $R \doteq \sup _{k}\left\|R_{\bullet}^{(k-1)}\right\|<\infty$ a.s., so using Proposition 6.5, for a.e. $\omega$, we can find some $\kappa_{4}(\omega) \in(0, \infty)$ such that

$$
\sigma_{n}^{1+\kappa}\left\|\sum_{k=1}^{n} \gamma_{k} \psi(n, k+1)\left(\boldsymbol{\mu}_{k-1}^{T} R_{\bullet}^{(k-1)} \boldsymbol{\mu}_{k-1}\right)\right\| \leq \kappa_{4} \sigma_{n}^{1+\kappa} \sum_{k=1}^{n} \gamma_{k} \exp \left(-L^{\prime} \sum_{j=k}^{n} \gamma_{j}\right)\left\|\boldsymbol{\mu}_{k-1}\right\|^{2}
$$


Using Proposition 6.4, we can find some $\kappa_{5} \in(0, \infty)$ such that for all $k \in \mathbb{N}, \mathbf{E}\left\|\boldsymbol{\mu}_{k-1}\right\|^{2} \leq \kappa_{5} \sigma_{k-1}^{2}$, so

$$
\sigma_{n}^{1+\kappa} \mathbf{E}\left[\sum_{k=1}^{n} \gamma_{k} \exp \left(-L^{\prime} \sum_{j=k}^{n} \gamma_{j}\right)\left\|\boldsymbol{\mu}_{k-1}\right\|^{2}\right] \leq \kappa_{5} \sigma_{n}^{1+\kappa} \sum_{k=1}^{n} \gamma_{k} \exp \left(-L^{\prime} \sum_{j=k}^{n} \gamma_{j}\right) \sigma_{k-1}^{2}
$$

From (6.17), we can bound the last term in (6.21) above by

$$
\kappa_{6} n^{\frac{1}{2}(1+\kappa)(1+\zeta)} \sum_{k=1}^{n} \gamma_{k} \exp \left(-L^{\prime} \sum_{j=k}^{n} \gamma_{j}\right)(k-1)^{-(1+\zeta)}
$$

for some $\kappa_{6} \in(0, \infty)$. Recalling that $\kappa<1 / 2$ we see that the expression in (6.22) converges to 0 as $n \rightarrow \infty$. Combining this observation with (6.20) and (6.21) we obtain (6.19). The result now follows on combining (6.15) and (6.19).

The next result will be used to prove Corollary 6.8 .

Proposition 6.7. Suppose that $\gamma_{*}>L^{-1}$. Then, as $n \rightarrow \infty$,

$$
\sigma_{n}\left[\sum_{k=1}^{n} \gamma_{k} \psi(n, k+1) \boldsymbol{r}_{k}\right] \stackrel{\mathbf{P}}{\rightarrow} 0
$$

Proof. Fix $L^{\prime} \in\left(\gamma_{*}^{-1}, L\right)$, and define

$$
\boldsymbol{r}_{n+1}^{(a)} \doteq \frac{1}{a(n+1)} \sum_{i=1}^{a(n+1)}\left(\left(K\left[\boldsymbol{\theta}_{n+1}\right] Q\left[\boldsymbol{\theta}_{n+1}\right]\right)_{\boldsymbol{X}_{n+1}^{i}, \cdot}-\left(K\left[\boldsymbol{\theta}_{n}\right] Q\left[\boldsymbol{\theta}_{n}\right]\right)_{\boldsymbol{X}_{n+1}^{i},}\right)
$$

and

$$
\boldsymbol{r}_{n+1}^{(b)} \doteq \frac{1}{a(n+1)} \sum_{i=1}^{a(n+1)}\left(\left(K\left[\boldsymbol{\theta}_{n}\right] Q\left[\boldsymbol{\theta}_{n}\right]\right)_{\boldsymbol{X}_{n}^{i}, \cdot}-\left(K\left[\boldsymbol{\theta}_{n+1}\right] Q\left[\boldsymbol{\theta}_{n+1}\right]\right)_{\boldsymbol{X}_{n+1}^{i}, .}\right)
$$

so that $\boldsymbol{r}_{n+1}=\boldsymbol{r}_{n+1}^{(a)}+\boldsymbol{r}_{n+1}^{(b)}$. Using Proposition 6.5 , we can find, for a.e. $\omega$, some $\kappa_{1}(\omega) \in(0, \infty)$ such that

$$
\left\|\sum_{k=1}^{n} \gamma_{k} \psi(n, k+1) \boldsymbol{r}_{k}^{(a)}\right\| \leq \kappa_{1} \sum_{k=1}^{n} \gamma_{k}^{2} \exp \left(L^{\prime} \sum_{j=k}^{n} \gamma_{j}\right)\left\|\frac{1}{\gamma_{k}} \boldsymbol{r}_{k}^{(a)}\right\|
$$

Using the fact that $\theta \rightarrow K[\theta], \theta \rightarrow Q[\theta]$ are bounded Lipschitz maps, we can find some $\kappa_{2} \in(0, \infty)$ such that for $k \in \mathbb{N}, \mathbf{E}\left\|\frac{1}{\gamma_{k}} \boldsymbol{r}_{k}^{(a)}\right\| \leq \kappa_{2}$. From this and (6.11), we can find some $\kappa_{3} \in(0, \infty)$ such that

$$
\sigma_{n} \sum_{k=1}^{n} \gamma_{k}^{2} \exp \left(-L^{\prime} \sum_{j=k}^{n} \gamma_{j}\right) \mathbf{E}\left\|\frac{1}{\gamma_{k}} \boldsymbol{r}_{k}^{(a)}\right\| \leq \kappa_{3} n^{\frac{1}{2}(1+\zeta)-L^{\prime} \gamma_{*}} \sum_{k=1}^{n} k^{L^{\prime} \gamma_{*}-2} .
$$


Since $\zeta<1$, the final term in (6.25) tends to 0 as $n \rightarrow \infty$. Combining this observation with (6.24) and (6.25), we have that, as $n \rightarrow \infty$,

$$
\sigma_{n}\left\|\sum_{k=1}^{n} \gamma_{k} \psi(n, k+1) \boldsymbol{r}_{k}^{(a)}\right\| \stackrel{\mathbf{P}}{\rightarrow} 0
$$

Next, for $n \in \mathbb{N}$ define

$$
\Xi_{n} \doteq \sum_{k=1}^{n} \boldsymbol{r}_{k}^{(b)}, \quad H_{n} \doteq \nabla h\left(\theta_{*}\right)+2 \boldsymbol{\mu}_{n}^{T} R_{\bullet}^{(n)}+\boldsymbol{\rho}_{n}^{T} R_{\bullet}^{(n)},
$$

and apply the summation by parts formula as in (4.18)-(4.19) to obtain

$$
\begin{aligned}
\sum_{k=1}^{n} \gamma_{k} \psi(n, k+1) \boldsymbol{r}_{k}^{(b)} & =\gamma_{n} \Xi_{n}-\sum_{k=1}^{n-1} \Xi_{k}\left(\gamma_{k+1} \psi(n, k+2)-\gamma_{k} \psi(n, k+1)\right) \\
& =\gamma_{n} \Xi_{n}+\sum_{k=1}^{n-1} \gamma_{k} \gamma_{k+1} \Xi_{k} \psi(n, k+2)\left(\gamma_{*}^{-1} I+H_{k}\right)
\end{aligned}
$$

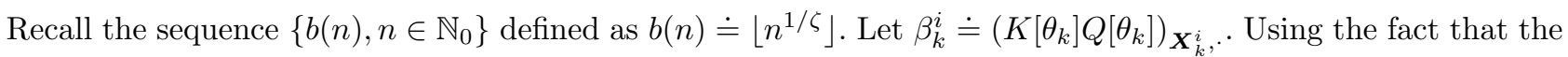
map $\theta \rightarrow\|K[\theta] Q[\theta]\|$ is bounded, we can find some $\kappa_{5} \in(0, \infty)$ such that

$$
\begin{array}{r}
\left\|\Xi_{n}\right\|=\left\|\sum_{k=1}^{n} \frac{1}{a(k)} \sum_{i=1}^{a(k)}\left(\beta_{k-1}^{i}-\beta_{k}^{i}\right)\right\|=\left\|\sum_{k=1}^{n} \sum_{i=2}^{a(n)} \frac{\beta_{k-1}^{i}-\beta_{k}^{i}}{a(k)} \mathbb{1}_{\{2 \leq i \leq a(k)\}}\right\|+\left\|\sum_{k=1}^{n} \frac{\beta_{k-1}^{1}-\beta_{k}^{1}}{a(k)}\right\| \\
\leq\left\|\sum_{k=1}^{n} \sum_{i=2}^{a(n)} \frac{\beta_{k-1}^{i}-\beta_{k}^{i}}{a(k)} \mathbb{1}_{\{2 \leq i \leq a(k)\}}\right\|+\left\|\frac{\beta_{0}^{1}}{a(1)}-\frac{\beta_{n}^{1}}{a(n)}\right\|+\left\|\sum_{k=1}^{n-1}\left(\frac{\beta_{k}^{1}}{a(k+1)}-\frac{\beta_{k}^{1}}{a(k)}\right)\right\| \\
\leq\left\|\sum_{k=1}^{n} \sum_{i=2}^{a(n)} \frac{\beta_{k-1}^{i}-\beta_{k}^{i}}{a(k)} \mathbb{1}_{\{2 \leq i \leq a(k)\}}\right\|+\kappa_{5} .
\end{array}
$$

Furthermore, there is a $\kappa_{6} \in(0, \infty)$ such that

$$
\begin{aligned}
&\left\|\sum_{i=2}^{a(n)} \sum_{k=1}^{n} \frac{\beta_{k-1}^{i}-\beta_{k}^{i}}{a(k)} \mathbb{1}_{\{2 \leq i \leq a(k)\}}\right\|=\left\|\sum_{i=2}^{a(n)} \sum_{k=b(i)}^{n} \frac{\beta_{k-1}^{i}-\beta_{k}^{i}}{a(k)}\right\| \\
&=\left\|\sum_{i=2}^{a(n)}\left[\frac{\beta_{b(i)-1}^{i}}{a(b(i))}+\sum_{k=b(i)}^{n-1}\left(\frac{\beta_{k}^{i}}{a(k+1)}-\frac{\beta_{k}^{i}}{a(k)}\right)-\frac{\beta_{n}^{i}}{a(n)}\right]\right\| \\
& \leq \kappa_{6} \sum_{i=2}^{a(n)}\left[\frac{1}{a(b(i))}+\sum_{k=b(i)}^{n-1}\left(\frac{1}{a(k)}-\frac{1}{a(k+1)}\right)+\frac{1}{a(n)}\right] \\
& \quad=\kappa_{6} \sum_{i=2}^{a(n)}\left[\frac{1}{a(b(i))}+\left(\frac{1}{a(b(i))}-\frac{1}{a(n)}\right)+\frac{1}{a(n)}\right]=2 \kappa_{6} \sum_{i=1}^{a(n)} \frac{1}{i} .
\end{aligned}
$$


Combining (6.28) and (6.29) we see that there is some $\kappa_{7} \in(0, \infty)$ such that for each $n \in \mathbb{N}$,

$$
\left\|\Xi_{n}\right\| \leq \kappa_{7} \log n
$$

Using (6.11) and (6.30), we can find some $\kappa_{8} \in(0, \infty)$ such that

$$
\sigma_{n} \gamma_{n}\left\|\Xi_{n}\right\| \leq \kappa_{8}(\log n) n^{-\frac{1}{2}(1-\zeta)}
$$

which tends to 0 as $n \rightarrow \infty$, since $\zeta<1$. Note that $A \doteq \sup _{n \in \mathbb{N}}\left\|\gamma_{*}^{-1} I+H_{n}\right\|<\infty$ a.s., which, along with (6.30) and Proposition 6.5, ensures that for a.e. $\omega$, there is some $\kappa_{9}(\omega) \in(0, \infty)$ such that

$$
\sigma_{n}\left\|\sum_{k=1}^{n-1} \gamma_{k} \gamma_{k+1} \Xi_{k} \psi(n, k+2)\left(\gamma_{*}^{-1} I+H_{k}\right)\right\| \leq \kappa_{9} \sigma_{n} \sum_{k=1}^{n-1} \gamma_{k}^{2} \exp \left(-L^{\prime} \sum_{j=k}^{n} \gamma_{j}\right) \log k
$$

The last term in $(6.32)$ can be bounded above by $\kappa_{10}(\log n) n^{-\frac{1}{2}(1-\zeta)}$, for some $\kappa_{10} \in(0, \infty)$ and hence the expression in (6.32) tends to 0 as $n \rightarrow \infty$. Combining this with (6.27) and (6.31), we see that as $n \rightarrow \infty$,

$$
\sigma_{n}\left\|\sum_{k=1}^{n} \gamma_{k} \psi(n, k+1) \boldsymbol{r}_{k}^{(b)}\right\| \stackrel{\mathbf{P}}{\rightarrow} 0
$$

The result follows on combining (6.26) and (6.33).

The following corollary says that the discrepancy sequence $\left\{\boldsymbol{\rho}_{n}\right\}$ tends to 0 in probability under the central limit scaling.

Corollary 6.8. Suppose that $\gamma_{*}>L^{-1}$. Then, as $n \rightarrow \infty, \sigma_{n} \boldsymbol{\rho}_{n} \stackrel{\mathbf{P}}{\rightarrow} 0$.

Proof. The result is immediate from Proposition 6.6 and Proposition 6.7.

\subsection{Proof of Theorem 1.5}

Consider the array $\left\{Z_{n, k}, n \in \mathbb{N}, 1 \leq k \leq n\right\}$ given by

$$
Z_{n, k} \doteq \sigma_{n} \gamma_{k} \psi_{*}(n, k+1) \boldsymbol{e}_{k}, \quad 1 \leq k \leq n, n \in \mathbb{N}
$$

Note that

$$
\sigma_{n} \boldsymbol{\mu}_{n}=\sum_{k=1}^{n} Z_{n, k}
$$

We will apply Corollary 3.1 of [23] to complete the proof of Theorem 1.5. The conditions for this result are verified in Lemma 6.9 and Lemma 6.10 given below.

Lemma 6.9. Suppose that $\gamma_{*}>L^{-1}$. Then, as $n \rightarrow \infty, \sum_{k=1}^{n+1} \mathbf{E}\left\|Z_{n+1, k}\right\|^{4} \rightarrow 0$.

Proof. Fix $L^{\prime} \in\left(\gamma_{*}^{-1}, L\right)$. Using (4.3), Proposition 6.2, and (6.11), we can find some $\kappa_{1} \in(0, \infty)$ such that

$$
\sum_{k=1}^{n} \mathbf{E}\left\|Z_{n, k}\right\|^{4}=\sum_{k=1}^{n} \mathbf{E}\left\|\sigma_{n} \gamma_{k} \psi_{*}(n, k+1) \boldsymbol{e}_{k}\right\|^{4} \leq \sigma_{n}^{4} \sum_{k=1}^{n} \gamma_{k}^{4}\left\|\psi_{*}(n, k+1)\right\|^{4} \mathbf{E}\left\|\boldsymbol{e}_{k}\right\|^{4}
$$




$$
\leq \kappa_{1} n^{2(1+\zeta)} \sum_{k=1}^{n} \gamma_{k}^{4} \exp \left(-4 L^{\prime} \sum_{j=k}^{n} \gamma_{j}\right) \frac{1}{a(k)^{2}}
$$

Thus, for some $\kappa_{2} \in(0, \infty)$ we have that

$$
\sum_{k=1}^{n} \mathbf{E}\left\|Z_{n, k}\right\|^{4} \leq \kappa_{2} n^{2(1+\zeta)-4 L^{\prime} \gamma_{*}} \sum_{k=1}^{n} k^{4\left(L^{\prime} \gamma_{*}-1\right)} k^{-2 \zeta},
$$

which tends to 0 as $n \rightarrow \infty$. The result follows.

The next lemma is used to characterize the limiting covariance matrix in Theorem 1.5. Recall the matrix $U_{*}$ defined in (3.2).

Lemma 6.10. Suppose that $\gamma_{*}>L^{-1}$. Define

$$
V_{n}^{(1)} \doteq \sigma_{n}^{2} \sum_{k=1}^{n} \gamma_{k}^{2} \psi_{*}(n, k+1) \frac{U_{*}}{a(k)} \psi_{*}(n, k+1)^{T},
$$

and

$$
V_{n}^{(2)} \doteq \sigma_{n}^{2} \sum_{k=1}^{n} \gamma_{k}^{2} \psi_{*}(n, k+1)\left[\mathbf{E}\left[\boldsymbol{e}_{k} \boldsymbol{e}_{k}^{T} \mid \mathcal{F}_{k-1}\right]-\frac{U_{*}}{a(k)}\right] \psi_{*}(n, k+1)^{T}
$$

As $n \rightarrow \infty, V_{n}^{(2)} \stackrel{\mathbf{P}}{\rightarrow} 0$ and $V_{n}^{(1)} \stackrel{P}{\rightarrow} \boldsymbol{V}$, where $\boldsymbol{V}$ is the unique solution of the Lyapunov equation

$$
U_{*}+(1+\zeta) \gamma_{*}^{-1} \boldsymbol{V}+\nabla h\left(\theta_{*}\right) \boldsymbol{V}+\boldsymbol{V} \nabla h\left(\theta_{*}\right)^{T}=0
$$

Proof. As in the proof of Lemma 4.5, the Lyapunov equation (6.37) has a unique solution, as $U_{*}$ is nonnegative definite, and the matrix

$$
\tilde{H} \doteq \nabla h\left(\theta_{*}\right)+(1+\zeta)\left(2 \gamma_{*}\right)^{-1} I
$$

is Hurwitz since $-L+(1+\zeta)\left(2 \gamma_{*}\right)^{-1}<-L+\gamma_{*}^{-1}<0$. We now consider $V_{n}^{(1)}$. Define $\tilde{\sigma}_{n}^{2} \doteq n^{-\zeta} \gamma_{n}$. Since $\tilde{\sigma}_{n} / \sigma_{n} \rightarrow 1$ as $n \rightarrow \infty$, it suffices to prove that

$$
\frac{\tilde{\sigma}_{n}^{2}}{\sigma_{n}^{2}} V_{n}^{(1)} \doteq \tilde{V}_{n}^{(1)} \stackrel{P}{\rightarrow} \boldsymbol{V}
$$

Observe that

$$
\frac{\tilde{\sigma}_{n+1}^{2}}{\tilde{\sigma}_{n}^{2}}-1=(1+\zeta) \frac{\gamma_{n}}{\gamma_{*}}+o\left(\gamma_{n}\right), \quad \frac{\tilde{\sigma}_{n}^{2}}{a(n)} \gamma_{n}^{2}=\gamma_{n}+o\left(\gamma_{n}\right)
$$

and therefore

$$
\frac{\tilde{\sigma}_{n+1}^{2}}{\tilde{\sigma}_{n}^{2}} \gamma_{n+1}^{2}=\gamma_{n+1}^{2}+o\left(\gamma_{n}^{2}\right), \quad \frac{\tilde{\sigma}_{n+1}^{2}}{\tilde{\sigma}_{n}^{2}} \gamma_{n+1}=\gamma_{n+1}+o\left(\gamma_{n}\right)
$$


From (4.1) it follows that

$$
\psi_{*}(n+1, k+1)=\left(I+\gamma_{n+1} \nabla h\left(\theta_{*}\right)\right) \psi_{*}(n, k+1)
$$

so we have that

$$
\begin{gathered}
\tilde{V}_{n+1}^{(1)}=\frac{\tilde{\sigma}_{n+1}^{2}}{a(n+1)} \gamma_{n+1}^{2} U_{*}+\frac{\tilde{\sigma}_{n+1}^{2}}{\tilde{\sigma}_{n}^{2}}\left(I+\gamma_{n+1} \nabla h\left(\theta_{*}\right)\right) \tilde{V}_{n}^{(1)}\left(I+\gamma_{n+1} \nabla h\left(\theta_{*}\right)\right)^{T} \\
=\frac{\tilde{\sigma}_{n+1}^{2}}{a(n+1)} \gamma_{n+1}^{2} U_{*}+\frac{\tilde{\sigma}_{n+1}^{2}}{\tilde{\sigma}_{n}^{2}}\left(\tilde{V}_{n}^{(1)}+\gamma_{n+1}\left[\nabla h\left(\theta_{*}\right) \tilde{V}_{n}^{(1)}+\tilde{V}_{n}^{(1)} \nabla h\left(\theta_{*}\right)^{T}\right]\right. \\
\left.+\gamma_{n+1}^{2} \nabla h\left(\theta_{*}\right) \tilde{V}_{n}^{(1)} \nabla h\left(\theta_{*}\right)^{T}\right) .
\end{gathered}
$$

Using the second identity in (6.40),

$$
\frac{\tilde{\sigma}_{n+1}^{2}}{a(n+1)} \gamma_{n+1}^{2} U_{*}=\gamma_{n+1} U_{*}+o\left(\gamma_{n}\right)
$$

Fix $L^{\prime} \in\left(\gamma_{*}^{-1}, L\right)$. Then, from (4.1) there is some $\kappa_{1} \in(0, \infty)$ such that

$$
\left\|\tilde{V}_{n}^{(1)}\right\| \leq \kappa_{1} n^{1+\zeta} \sum_{k=1}^{n} \gamma_{k}^{2} \exp \left(-2 L^{\prime} \sum_{j=k+1}^{n} \gamma_{j}\right) k^{-\zeta},
$$

and so we can find some $\kappa_{2} \in(0, \infty)$ such that

$$
\left\|\tilde{V}_{n}^{(1)}\right\| \leq \kappa_{2}, \quad n \in \mathbb{N}
$$

Combining the first identity in (6.40) with (6.44) we see that

$$
\frac{\tilde{\sigma}_{n+1}^{2}}{\tilde{\sigma}_{n}^{2}} \tilde{V}_{n}^{(1)}-\tilde{V}_{n}^{(1)}=\left(\frac{\tilde{\sigma}_{n+1}^{2}}{\tilde{\sigma}_{n}^{2}}-1\right) \tilde{V}_{n}^{(1)}=(1+\zeta) \frac{\gamma_{n}}{\gamma_{*}} \tilde{V}_{n}^{(1)}+o\left(\gamma_{n}\right) .
$$

Additionally, from (6.41) and (6.44) we see that

$$
\frac{\tilde{\sigma}_{n+1}^{2}}{\tilde{\sigma}_{n}^{2}} \gamma_{n+1}^{2} \nabla h\left(\theta_{*}\right) \tilde{V}_{n}^{(1)} \nabla h\left(\theta_{*}\right)^{T}=o\left(\gamma_{n}\right) .
$$

Finally, noting that $\gamma_{n+1}=\gamma_{n}+o\left(\gamma_{n}\right)$, and combining (6.41), (6.43), (6.45), and (6.46) with (6.42) we see that

$$
\tilde{V}_{n+1}^{(1)}=\tilde{V}_{n}^{(1)}+\gamma_{n}\left[U_{*}+\gamma_{*}^{-1}(1+\zeta) \tilde{V}_{n}^{(1)}+\nabla h\left(\theta_{*}\right) \tilde{V}_{n}^{(1)}+\tilde{V}_{n}^{(1)} \nabla h\left(\theta_{*}\right)^{T}\right]+o\left(\gamma_{n}\right) .
$$

Let $\boldsymbol{V}$ denote the aforementioned unique solution to (6.37). Recall from (6.37) that the matrix $\tilde{H}$ defined in (6.38) satisfies $U_{*}+\tilde{H} \boldsymbol{V}+\boldsymbol{V} \tilde{H}^{T}=0$. Thus, (6.47) can be rewritten as

$$
\tilde{V}_{n+1}^{(1)}-\boldsymbol{V}=\tilde{V}_{n}^{(1)}-\boldsymbol{V}+\gamma_{n}\left[\tilde{H}\left(\tilde{V}_{n}^{(1)}-\boldsymbol{V}\right)+\left(\tilde{V}_{n}^{(1)}-\boldsymbol{V}\right) \tilde{H}^{T}\right]+o\left(\gamma_{n}\right) .
$$


Recalling once more that $\tilde{H}$ is Hurwitz, it follows from (6.48) and the proof of Lemma 5.11 in [21] that $\tilde{V}_{n}^{(1)} \stackrel{\mathbf{P}}{\rightarrow} \boldsymbol{V}$ as $n \rightarrow \infty$. This proves (6.39) and, as noted previously, shows that $V_{n}^{(1)} \stackrel{\mathbf{P}}{\rightarrow} \boldsymbol{V}$ as $n \rightarrow \infty$. The proof that $V_{n}^{(2)} \stackrel{\mathbf{P}}{\rightarrow} 0$ as $n \rightarrow \infty$ is similar to the analogous result in Lemma 4.5, and is omitted.

Proof of Theorem 1.5. From (1.26) we see that $\sigma_{n}\left(\boldsymbol{\theta}_{n}-\theta_{*}\right)=\sigma_{n} \boldsymbol{\mu}_{n}+\sigma_{n} \boldsymbol{\rho}_{n}$. Also, from Corollary $6.8, \sigma_{n} \boldsymbol{\rho}_{n} \stackrel{\text { P }}{\rightarrow} 0$ as $n \rightarrow \infty$. Thus, it suffices to show that $\sigma_{n} \boldsymbol{\mu}_{n} \stackrel{\mathcal{L}}{\rightarrow} \mathcal{N}(0, \boldsymbol{V})$ where $V$ is as in the statement of the theorem. Recall the martingale difference array $\left\{Z_{n, k}\right\}$ introduced in (6.34), and note from (6.35) that $\sigma_{n} \boldsymbol{\mu}_{n}=\sum_{k=1}^{n} Z_{n, k}$. In order to complete the proof we apply Corollary 3.1 to [23]. From Lemma 4.4 it follows that for each $\epsilon>0$,

$$
\sum_{k=1}^{n} \mathbf{E}\left[\left\|Z_{n, k}\right\|^{2} \mathbb{1}_{\left\|Z_{n, k}\right\| \geq \epsilon} \mid \mathcal{F}_{k-1}\right] \stackrel{\mathbf{P}}{\rightarrow} 0,
$$

as $n \rightarrow \infty$. Additionally, if we let $\left\{V_{n}^{(1)}\right\},\left\{V_{n}^{(2)}\right\}$, and $\boldsymbol{V}$ be as in Lemma 6.10 , then we have that

$$
\sum_{k=1}^{n} \mathbf{E}\left[Z_{n, k} Z_{n, k}^{T} \mid \mathcal{F}_{k-1}\right]=V_{n}^{(1)}+V_{n}^{(2)}
$$

and from Lemma $6.10, \sum_{k=1}^{n} \mathbf{E}\left[Z_{n, k} Z_{n, k}^{T} \mid \mathcal{F}_{k-1}\right] \stackrel{\mathbf{P}}{\rightarrow} \boldsymbol{V}$, as $n \rightarrow \infty$. Therefore, the conditions of Corollary $3.1 \mathrm{in}$ [23] are satisfied, proving that $\sum_{k=1}^{n} Z_{n, k} \stackrel{\mathcal{L}}{\rightarrow} \mathcal{N}(0, \boldsymbol{V})$, as $n \rightarrow \infty$. The result follows.

\section{NumERICAL EXPERIMENTS}

In this section we present results from some numerical experiments. We compare five simulation based methods for computing the QSD of a finite state Markov chain. The first four methods can be viewed as stochastic approximation algorithms and are described in terms of a sequence of step sizes given as

$$
\gamma_{n+1} \doteq \frac{\gamma_{*}}{n+N_{*}}, \quad n \in \mathbb{N}_{0},
$$

where $\gamma_{*} \in(0, \infty)$ and $N_{*} \doteq\left\lfloor\gamma_{*}\right\rfloor+1$. In order to ensure that the results from the various methods are comparable, we measure the run-time of each method by the total number of particle transitions.

The first estimation method, which we refer to as the Single scheme, is the algorithm given in equation (7) of [6]. In order to obtain an estimate for the QSD using this scheme, we run the algorithm for

$$
\xi(n) \doteq n a(n)
$$

time steps. Since there is a single particle, and it moves once at each time step, this means that there are a total of $\xi(n)$ particle transitions. The second scheme, which we refer to as the Independent scheme, is given by evolving $a(n)$ Single schemes independently of one another. Each of these independent schemes runs for $n$ time steps, and the estimate for the QSD is then given by the average of the $a(n)$ estimates. At each time instant, there are $a(n)$ particle transitions, so the total number of particle transitions is $\xi(n)$. The third scheme, which we refer to as the Interacting scheme, is the algorithm defined in (1.4). In the notation of this work, our final estimate for the QSD is then given by $\theta_{n}^{n}$. As with the Independent scheme, since $a(n)$ particles move at each time instant, there are $\xi(n)$ particle transitions in total, and $\xi(n)$ is again given by (7.1). The fourth scheme is the Branching scheme, which is described in (1.8). Note that for this scheme, by time instant $k$ there are a total of $\sum_{i=1}^{k} a(i+1)$ particle transitions. Consequently, we run this scheme for $\xi_{B}(n)$ time steps, where 


$$
\xi_{B}(n) \doteq \inf \left\{k: \sum_{i=1}^{k} a(i+1) \geq n a(n)\right\}
$$

The final method is the Fleming-Viot approximation. A description of this method and some important results regarding its convergence properties can be found in [22]. In order to estimate the QSD using the FlemingViot approximation, we consider a collection of $v(n)$ particles that evolve according to the dynamics described in [22]. At each time instant a particle is chosen uniformly at random to move, so after $n v(n)$ time steps, there have been $n v(n)$ particle transitions. The final estimate for the QSD is given by the empirical measure of the $v(n)$ particles at the $n v(n)$-th time instant. In light of the convergence results regarding the FlemingViot approximation established in e.g., [22], we consider situations in which the number of particles grows exponentially with time, namely the setting where the Fleming-Viot approximation method runs for $\xi_{F V}(n)$ time steps, where $\xi_{F V}(n) \approx\lfloor\exp (n)\rfloor n$.

Our numerical results are exploratory in nature, and are intended as a starting point for a more detailed comparison of the performance of the aforementioned approximation methods. We also explore the impact of several choices that affect the performance of each algorithm, such as the initial distribution of the particles in the system and the relationship between the number of particles and the algorithms' overall performance. The experimental results suggest that the methods all converge rapidly when the dynamics of the underlying Markov chain are relatively simple. For example, for the Markov chain whose transition matrix is of the form

$$
P=\left[\begin{array}{ccc}
1 & 0 & 0 \\
1-\epsilon_{1}-\epsilon_{2} & \epsilon_{1} & \epsilon_{2} \\
1-\epsilon_{3}-\epsilon_{4} & \epsilon_{3} & \epsilon_{4}
\end{array}\right]
$$

where $\epsilon_{1}, \epsilon_{2}, \epsilon_{3}, \epsilon_{4} \in(0,1)$ satisfy $\epsilon_{1}+\epsilon_{2}<1$ and $\epsilon_{3}+\epsilon_{4}<1$, the rates of convergence of the various methods were comparable regardless of the distribution of the initial states of the particles in the systems.

However, more significant differences begin to emerge when there are several points in the state space at which the underlying Markov chain is expected to spend a relatively long time. In our experiments, systems with several of these metastable states tended to favor approximation methods consisting of many (interacting) particles. This is consistent with our intuition, as the presence of multiple metastable states may lead to individual particles becoming trapped for long periods of time. For a Markov chain $\left\{Y_{n}\right\}$ on $\Delta$, we refer to a point $x \in \Delta^{o}$ as a basin if $\mathbf{E}_{x}\left(Y_{1}\right)=x$.

We now consider two examples of Markov chains that have several basins. The first chain is the Markov chain $\left\{Y_{n}\right\}$ on $\Delta \doteq\{0,1, \ldots, 9\}$ with transition probability matrix $P_{1}$ given below in (7.2). Note that $\left\{Y_{n}\right\}$ has three basins, namely, 2,5, and 8. Applying Corollary 2.3 of [6] we see that $\gamma_{*} \approx 4.17$ satisfies $\gamma_{*}>L^{-1}$, where $L$ is as in Section 1.1. The second chain is the Markov chain $\left\{Z_{n}\right\}$ on $\Delta \doteq\{0,1, \ldots, 7\}$ with transition probability matrix $P_{2}$ given in (7.2). Here $\left\{Z_{n}\right\}$ has three basins, namely, 2, 4, and 6 . As with $P_{1}$ above, we applied Corollary 2.3 of [6] to see that $\gamma_{*} \approx 2.54$ satisfies $\gamma_{*}>L^{-1}$, where $L$ is as in Section 1.1. While the chain with transitions matrix $P_{1}$ and the chain with transitions matrix $P_{2}$ both have three basins, one should expect that approximation methods will generally converge more quickly when estimating the QSD of $P_{2}$, due to the fact that the state space of the underlying chain is smaller, and therefore that the particles may traverse the state space more quickly.

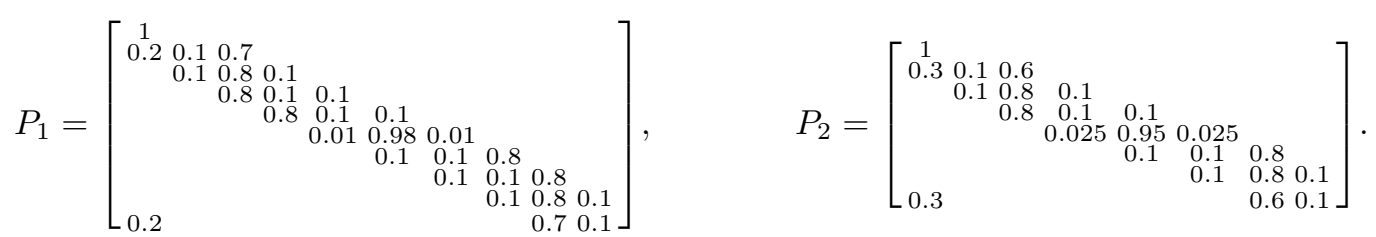




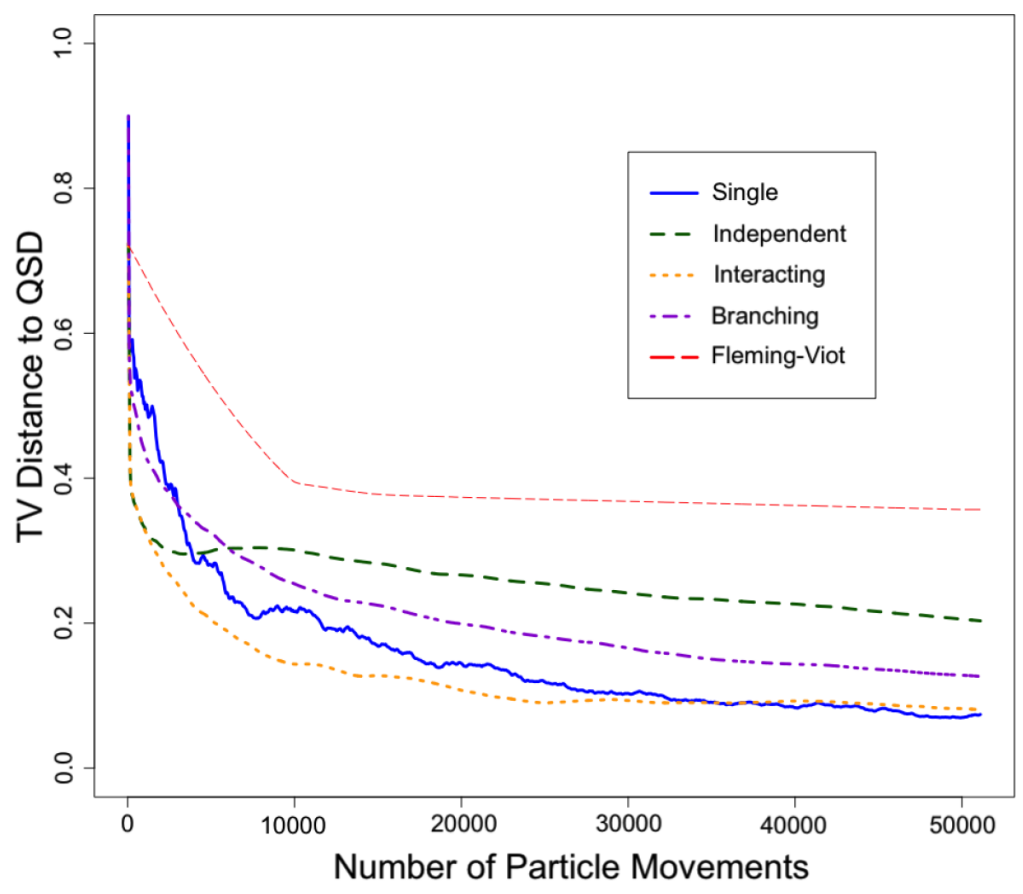

Figure 1. Experimental results for the $P_{1}$ chain. Here $a(n)=\left\lfloor n^{0.75}\right\rfloor$ and $n \doteq 1000$.

The remainder of Section 7 is organized as follows. In Section 7.1 we compare the performance of the five approximation methods when approximating the QSD of the Markov chain with transition matrix $P_{1}$. These results suggest that the Interacting Scheme may outperform the Independent, Single, and Branching Schemes when the underlying chain contains several basins at which the particles tend to spend long periods of time. In Section 7.2 we compare the Interacting Scheme and the Fleming-Viot Approximation method by investigating each algorithm's performance in several settings. Similarly, in Section 7.3 we compare the Interacting Scheme and the Single Scheme, and in Section 7.4 we compare the Interacting Scheme and the Independent Scheme. Finally, in Section 7.5 we investigate the impact of the choice of the sequence $a(n)$ on the Interacting Scheme.

\subsection{Comparing the algorithms}

Recall the transition matrix $P_{1}$ defined in (7.2). For the experimental setup (A) we take $a(n)=\left\lfloor n^{0.75}\right\rfloor$ and $n \doteq 1000$. We repeated the experiment for each scheme $R=300$ times and averaged the results. For the Independent, Interacting, and Fleming-Viot schemes, the initial states of the $a(1000)=177$ particles were chosen uniformly at random from $\{4,5,6\}$. This same set of initial states was used in each of the 300 repetitions of the simulation. Since the Single and Branching schemes are initialized with only a single particle, we chose the initial states of the 300 repetitions so that they would be proportionate to the initial states used for the schemes that start with $a(n)$ particles. In Figure 1 we plot the total variation distance between the estimate of the QSD given by each scheme and the true QSD as a function of the number of particle transitions. The results are plotted for the first 70,000 particle transitions.

Note that the Interacting scheme converges most quickly to the QSD in this experiment. The Fleming-Viot algorithm appears to have a significant asymptotic bias, which is a consequence of the fact that the number of particles is not sufficiently large for the time asymptotic behavior of the Fleming-Viot processes to effectively approximate the QSD. The experimental results when the initial states of the particles were chosen uniformly at random from $\Delta^{o}$ were similar. 
TABLE 1. Total variation distance from the interacting and Fleming-Viot approximations to the QSD of $P_{1}$. For the interacting scheme, $n \doteq 500$, and $a(n)$ varies. For the Fleming-Viot approximation, we allowed for different choices of $n$, and each system had $n v(n)$ particle transitions. Initial states were chosen uniformly over the described sets.

\begin{tabular}{|c|c|c|c|c|c|c|c|c|c|}
\hline \multicolumn{2}{|c|}{ Particle transitions } & 5500 & 10500 & 15500 & 20500 & 5500 & 10500 & 15500 & 20500 \\
\hline \multirow{2}{*}{\multicolumn{2}{|c|}{$\begin{array}{l}a(n) \text { or } v(n) \\
\text { Method }\end{array}$}} & 11 & 21 & 31 & 41 & 819 & 1443 & 2034 & 2606 \\
\hline & & \multicolumn{4}{|c|}{ Interacting } & \multicolumn{4}{|c|}{ Fleming-Viot } \\
\hline \multirow{3}{*}{ Initial states } & 4 & 0.0823 & 0.0619 & 0.0508 & 0.0517 & 0.4707 & 0.4542 & 0.4435 & 0.4380 \\
\hline & $\{3,4$, $\}$ & 0.0963 & 0.0714 & 0.0496 & 0.0511 & 0.0917 & 0.1247 & 0.0735 & 0.1885 \\
\hline & all 2 & 0.0988 & 0.0643 & 0.0622 & 0.0491 & 0.0552 & 0.2059 & 0.0773 & 0.1228 \\
\hline
\end{tabular}

TABLE 2. Total variation distance from the interacting and Fleming-Viot approximations to the QSD of $P_{2}$. For the interacting scheme, $n \doteq 1000$, and $a(n)$ varies. For the Fleming-Viot approximation, we allowed for different choices of $n$, and each system had $n v(n)$ particle transitions. Initial states were chosen uniformly over the described sets.

\begin{tabular}{|c|c|c|c|c|c|c|c|c|c|c|c|}
\hline \multicolumn{2}{|c|}{ Particle transitions } & 6000 & 11000 & 16000 & 21000 & 26000 & 6000 & 11000 & 16000 & 21000 & 26000 \\
\hline \multicolumn{2}{|l|}{$a(n)$ or $v(n)$} & 6 & 11 & 16 & 21 & 26 & 884 & 1503 & 2092 & 2662 & 3219 \\
\hline \multirow{2}{*}{\multicolumn{2}{|c|}{ Method }} & \multicolumn{5}{|c|}{ Interacting } & \multicolumn{5}{|c|}{ Fleming-Viot } \\
\hline & & 0.2748 & 0.2453 & 0.2058 & 0.1670 & 0.1326 & 0.4973 & 0.4936 & 0.4958 & 0.4939 & 0.4960 \\
\hline Initial states & $\{4,5,6\}$ & 0.2753 & 0.1478 & 0.1605 & 0.1225 & 0.1117 & 0.5469 & 0.3798 & 0.3598 & 0.3522 & 0.3556 \\
\hline & all $\Delta^{o}$ & 0.2767 & 0.1578 & 0.1307 & 0.1322 & 0.1243 & 0.0922 & 0.2118 & 0.1650 & 0.1409 & 0.3255 \\
\hline
\end{tabular}

\subsection{Interacting scheme and Fleming-Viot approximation}

In order to compare the performance of the Interacting Scheme and the Fleming-Viot Approximation, we used both methods to approximate the QSD of $P_{1}$ and $P_{2}$. To ensure the Fleming-Viot approximation was initialized with sufficiently many particles to obtain an accurate approximation, in each of our experiments we let the number of particles driving the approximation grow exponentially with time $(n)$. The number of particles driving the Fleming-Viot approximation is denoted by $v(n)$. To obtain the comparisons presented in this section, we measured the algorithms' performance for several different choices of total particle transitions. Recall that (for a particular choice of the $a(n)$ sequence) $\xi(n)$ denotes the number of particle transitions in the Interacting (and Independent) Scheme in $n$ time instants, and that $\xi_{F V}(n)$ denotes the number of particle movements in the Fleming-Viot particle system in $n$ time instants, where the number of particles scales exponentially with time.

The results of these experiments are presented in Tables 1 and 2. The entries in the tables are as follows: the first row gives the total number of particle transitions in each system, namely $\xi_{F V}(n)=\xi(n)$. The next row describes the number of particles driving the dynamics of the Interacting Scheme, namely $a(n)$, and the Fleming-Viot method, $v(n)$, for each choice of total particle transitions. The next three rows describes the total variation distance of the Interacting Scheme and the Fleming-Viot approximations to the QSD after the aforementioned number of particle transitions.

While these comparisons are exploratory in nature, several key trends became apparent. The first is that even though both the Interacting Scheme and the Fleming-Viot Approximation are sensitive to the choice of initial conditions, the Fleming-Viot Approximation may be even more so. This coincides with our expectations, as the Fleming-Viot Approximation's dynamics are driven by a large number of particles, so if the particles all start near a highly-attractive basin of the underlying Markov chain, then the time for the particles to thoroughly explore the state space may tend to be quite large. A second point is that for both approximation methods, when the number of time steps is fixed, if the number of particles in each system is increased, the resultant approximation does not always improve (although for the Interacting Scheme it typically does). 
TABLE 3. Total variation distance from the Interacting and Single Schemes to the QSD of $P_{1}$. For the interacting scheme, $n \doteq 300$, and $a(n)$ varies. The single scheme is ran for $n a(n)$ particle transitions. The initial state of the single particle was 4 , and the interacting particles were started uniformly in $\Delta^{o}$.

\begin{tabular}{lccccccc}
\hline Particle transitions & 5000 & 9000 & 13000 & 17000 & 21000 & 25000 & 29000 \\
\hline$a(n)$ & 17 & 30 & 44 & 57 & 70 & 84 & 97 \\
Interacting & 0.1713 & 0.1551 & 0.1450 & 0.1060 & 0.1048 & 0.0680 & 0.0580 \\
Single & 0.2701 & 0.2516 & 0.1820 & 0.2139 & 0.1322 & 0.1090 & 0.1412 \\
\hline
\end{tabular}

TABLE 4. The total variation distance from the Interacting Scheme and the Single Scheme to the QSD of $P_{2}$. For the interacting scheme, $n \doteq 2000$, and $a(n)$ varies. For each choice of $a(n)$, the single scheme is ran for $n a(n)$ particle transitions. For these experiments, the initial state of the particle in the single scheme was state 5, and the particles in the Interacting Scheme started at state 5 .

\begin{tabular}{lccccc}
\hline Particle transitions & 5000 & 21000 & 37000 & 57000 & 85000 \\
\hline$a(n)$ & 3 & 11 & 19 & 29 & 43 \\
Interacting & 0.2615 & 0.1215 & 0.0782 & 0.0752 & 0.0460 \\
Single & 0.3352 & 0.1996 & 0.1034 & 0.0909 & 0.0722 \\
\hline
\end{tabular}

\subsection{Interacting scheme and single scheme}

Our next set of experiments compares the performance of the Interacting Scheme and the Single Scheme. For these comparisons, the two approximation methods were both run for $\xi(n)$ particle movements. The results appear in Tables 3 and 4. Our experiments yielded two key observations regarding the performance of these two approximation methods. The first is that, in line with our expectations, the Interacting Scheme may be more well-suited for approximating the QSD of Markov chains with several basins, as the particle driving the Single Scheme's approximation may become trapped near such points for long periods of time. In contrast with this, even if some of the particles driving the Interacting Scheme's approximation become trapped near basins of the underlying chain, the other particles driving the approximation can still explore the state space. The interactions between the particles then allow even the particles which are trapped near basins to continue updating their dynamics according to the other particles, which may be moving more freely throughout the system. Our second key observation is that in state spaces with multiple basins, we generally expect that it will continue to be advantageous to distribute the particles driving the Interacting Scheme's approximation throughout the system, which, of course, is not possible with the Single Scheme.

\subsection{Interacting scheme and independent scheme}

Unlike the Fleming-Viot approximation and the Single Scheme, the Independent Scheme is directly comparable to the Interacting Scheme in terms of the number of particles in the system and the impact of the initial distribution of the particles. Accordingly, the comparison of the two algorithms is relatively straightforward. The results are in Tables 5 and 6, and again suggest that for systems in which there are multiple basins at which particles may become stuck for long periods of time, the Interacting Scheme may provide a more accurate approximation. Our intuition behind this performance disparity is that even if some of the particles driving the Independent Scheme become trapped around basins, they can continue to update their dynamics according to the other particles' positions. While the difference in performance between these two methods tended to be relatively minor, we expect that in more complicated settings (e.g., larger state spaces with more basins), that the Interacting Scheme would continue to outperform the Independent Scheme. 
TABLE 5. Total variation distance for Interacting and Independent schemes to the QSD of $P_{1}$. In both schemes, $n \doteq 2000$, and $a(n)$ varies. All particles started from state 5 .

\begin{tabular}{lccccccc}
\hline Particle transitions & 4000 & 8000 & 16000 & 24000 & 32000 & 40000 & 48000 \\
\hline$a(n)$ & 2 & 4 & 8 & 12 & 16 & 20 & 24 \\
Interacting & 0.3191 & 0.2032 & 0.1079 & 0.1081 & 0.0880 & 0.0845 & 0.0841 \\
Independent & 0.2450 & 0.2067 & 0.1363 & 0.1654 & 0.1375 & 0.1120 & 0.1018 \\
\hline
\end{tabular}

TABLE 6. Total variation distance for Interacting and Independent schemes to the QSD of $P_{2}$. In both schemes, $n \doteq 2000$, and $a(n)$ varies.

\begin{tabular}{|c|c|c|c|c|c|c|c|c|c|c|}
\hline \multicolumn{3}{|c|}{ Particle transitions } & 15000 & 25000 & 35000 & 45000 & 55000 & 65000 & 75000 & 85000 \\
\hline \multicolumn{3}{|c|}{$a(n)$} & 8 & 13 & 18 & 23 & 28 & 33 & 38 & 43 \\
\hline \multirow{2}{*}{ Interacting } & \multirow{4}{*}{ Initial states } & 5 & 0.1231 & 0.1041 & 0.1027 & 0.0568 & 0.0835 & 0.0738 & 0.0469 & 0.0568 \\
\hline & & $4,5,6\}$ & 0.1482 & 0.0824 & 0.0913 & 0.0817 & 0.0627 & 0.0665 & 0.0642 & 0.0571 \\
\hline \multirow{2}{*}{ Independent } & & & 0.1251 & 0.1162 & 0.1117 & 0.1117 & 0.1035 & 0.1054 & 0.1050 & 0.1056 \\
\hline & & $\{4,5,6\}$ & 0.1382 & 0.1287 & 0.1355 & 0.1288 & 0.1063 & 0.1270 & 0.1069 & 0.1133 \\
\hline
\end{tabular}

TABLE 7. Total variation distance from the Interacting Scheme to the QSD of $P_{2}$ after 10000 particle movements for various choices of $a(n)$.

\begin{tabular}{|c|c|c|c|c|c|c|c|c|c|c|}
\hline \multirow{3}{*}{$\begin{array}{l}n \\
a(n)\end{array}$} & & 100 & 200 & 300 & 400 & 500 & 800 & 1300 & 2000 & 10000 \\
\hline & & 100 & 50 & 34 & 25 & 20 & 13 & 8 & 5 & 1 \\
\hline & 5 & 0.2144 & 0.1359 & 0.1138 & 0.1210 & 0.1412 & 0.1335 & 0.1543 & 0.1578 & 0.1810 \\
\hline \multirow[t]{2}{*}{ Initial states } & $\{4,5,6\}$ & 0.0965 & 0.1052 & 0.1221 & 0.1106 & 0.1389 & 0.1499 & 0.1585 & 0.1578 & 0.2417 \\
\hline & & 0.0852 & 0.0981 & 0.1096 & 0.1213 & 0.1572 & 0.1627 & 0.1684 & 0.2218 & 0.2103 \\
\hline
\end{tabular}

\subsection{Selecting the number of particles in the interacting scheme}

The experiments presented here are intended to explore the impact that the choice of the sequence $a(n)$ has on the performance of the Interacting Scheme. To study the effect of $a(n)$, we fixed a total number of particle movements $\xi(n)$ and compared the performance for various choices of $a(n)$. The results are presented in Table 7. The experiments suggested that, at least up to a point, it may be advantageous to increase the number of particles in the system, rather than solely increasing amount of time.

\section{Appendix A. A matrix estimate}

The following lemma is similar to Lemma 5.8 of [21].

Lemma A.1. Let $A$ be a $d \times d$ Hurwitz matrix such that the real part of all of its eigenvalues is bounded above by $-L$ where $L \in(0, \infty)$. Fix $p \in(0,1)$. Let $\left\{A_{k}^{n}\right\}_{n, k=1}^{\infty}$ be an array of matrices such that $\sup _{n^{p} \leq k \leq n}\left\|A_{k}^{n}-A\right\| \rightarrow 0$, where $\|\cdot\|$ denotes the Frobenius norm on the space of $d \times d$ matrices. For each $L^{\prime} \in(0, L)$, there is a constant $C>0$ such that if $n^{p} \leq k \leq n$, then

$$
\left\|\prod_{j=k}^{n}\left(I+\gamma_{j} A_{j}^{n}\right)\right\| \leq C \exp \left(-L^{\prime} \sum_{j=k}^{n} \gamma_{j}\right)
$$

Proof. Let $\left\{\lambda_{i}\right\}_{i=1}^{d}$ denote the eigenvalues of $A$, and use the Jordan decomposition of $A$ to write $A=S J S^{-1}$, where $S$ is invertible and $J$ is a Jordan matrix. Let

$$
D_{t} \doteq \operatorname{diag}\left(t, t^{2}, \ldots, t^{d}\right), \quad \Lambda \doteq \operatorname{diag}\left(\lambda_{1}, \lambda_{2}, \ldots, \lambda_{d}\right)
$$


Then, following [21], we have that $A=\left(S D_{t}\right)\left(\Lambda+R_{t}\right)\left(S D_{t}\right)^{-1}$, where $\lim _{t \rightarrow 0}\left\|R_{t}\right\|=0$. Write

$$
\left(S D_{t}\right)^{-1}\left(I+\gamma_{j} A_{j}^{n}\right)\left(S D_{t}\right)=I+\gamma_{j} \Lambda+\gamma_{j} R_{t}+\gamma_{j}\left(S D_{t}\right)^{-1}\left(A_{j}^{n}-A\right)\left(S D_{t}\right) .
$$

For $n^{p} \leq k \leq n$, we have

$$
\left\|\left(S D_{t}\right)^{-1}\left(I+\gamma_{k} A_{k}^{n}\right)\left(S D_{t}\right)\right\| \leq\left\|I+\gamma_{k} \Lambda\right\|+\gamma_{k}\left\|R_{t}\right\|+\gamma_{k}\left\|A_{k}^{n}-A\right\|\left\|S D_{t}\right\|\left\|\left(S D_{t}\right)^{-1}\right\| .
$$

Fix $0<L^{\prime}<L^{\prime \prime}<L$, and note that there is some $t_{0}>0$ such that if $0 \leq t \leq t_{0}$, then $\left\|R_{t}\right\| \leq\left(L^{\prime \prime}-L^{\prime}\right) / 2$. Also, there is some $n_{0}$ such that if $n \geq n_{0}, n^{p} \leq k \leq n$, then

$$
\left\|I+\gamma_{k} \Lambda\right\| \leq 1-\gamma_{k} L^{\prime \prime}, \quad\left\|A_{k}^{n}-A\right\|\left\|S D_{t}\right\|\left\|\left(S D_{t}\right)^{-1}\right\| \leq\left(L^{\prime \prime}-L^{\prime}\right) / 2 .
$$

Combining (A.1) and (A.2), we see that if $n^{p} \leq k \leq n, t \leq t_{0}$, and $n \geq n_{0}$,

$$
\left\|\left(S D_{t}\right)^{-1}\left(I+\gamma_{k} A_{k}^{n}\right)\left(S D_{t}\right)\right\| \leq 1-\gamma_{k} L^{\prime}
$$

It follows that

$$
\begin{aligned}
\left\|\prod_{j=k}^{n}\left(I+\gamma_{j} A_{j}^{n}\right)\right\| & =\left\|\left(S D_{t}\right)\left[\prod_{j=k}^{n}\left(S D_{t}\right)^{-1}\left(I+\gamma_{j} A_{j}^{n}\right)\left(S D_{t}\right)\right]\left(S D_{t}\right)^{-1}\right\| \\
& \leq\left\|S D_{t}\right\| \prod_{j=k}^{n}\left\|\left(S D_{t}\right)^{-1}\left(I+\gamma_{j} A_{j}^{n}\right)\left(S D_{t}\right)\right\|\left\|\left(S D_{t}\right)^{-1}\right\| \\
& \leq\left\|S D_{t}\right\|\left\|\left(S D_{t}\right)^{-1}\right\| \exp \left(-L^{\prime} \sum_{j=k}^{n} \gamma_{j}\right) .
\end{aligned}
$$

Acknowledgements. Research of AB is supported in part by the National Science Foundation (DMS-1814894 and DMS1853968). AB is grateful for the support from Nelder Fellowship from Imperial College, London, where part of this research was completed.

\section{REFERENCES}

[1] D. Aldous, B. Flannery and J.L. Palacios, Two applications of urn processes: the fringe analysis of search trees and the simulation of quasi-stationary distributions of Markov chains. Prob. Eng. Inform. Sci. 2 (1988) 293-307.

[2] M.S. Bartlett, Methuen's monographs on applied probability and statistics. Methuen (1960).

[3] M. Benaïm, Vertex-reinforced random walks and a conjecture of Pemantle. Ann. Probab. 25 (1997) 361-392.

[4] M. Benaïm, Dynamics of stochastic approximation algorithms. Séminaire de probabilités, XXXIII 1709 (1999) 1-68.

[5] M. Benaïm, N. Champagnat and D. Villemonais, Stochastic approximation of quasi-stationary distributions for diffusion processes in a bounded domain. Ann. l'Inst. Henri Poincaré, Prob. Stat. 57 (2021) 726-739.

[6] M. Benaïm and B. Cloez, A stochastic approximation approach to quasi-stationary distributions on finite spaces. Electron. Commun. Probab. 20 (2015) 1-13.

[7] M. Benaïm, B. Cloez and F. Panloup, Stochastic approximation of quasi-stationary distributions on compact spaces and applications. Ann. Appl. Prob. 28 (2016).

[8] M. Benaïm and M. Hirsch, Asymptotic pseudotrajectories and chain recurrent flows, with applications. J. Dyn. Differ. Equ. 8 (1996) $141-176$.

[9] A. Benveniste, M. Métivier and P. Priouret, Vol. 22 of Adaptive Algorithms and Stochastic Approximations. Springer Science \& Business Media (2012).

[10] J. Blanchet, P. Glynn and S. Zheng, Analysis of a stochastic approximation algorithm for computing quasi-stationary distributions. Adv. Appl. Prob. 48 (2016) 792-811.

[11] V.S. Borkar, Vol. 48 of Stochastic Approximation: A Dynamical Systems Viewpoint. Springer (2009). 
[12] K. Burdzy, R. Holyst and P. March, A Fleming-Viot particle representation of the Dirichlet Laplacian. Commun. Math. Phys. 214 (2000) 679-703.

[13] F. Cérou, B. Delyon, A. Guyader and M. Rousset, A central limit theorem for Fleming-Viot particle systems. Ann. l'Inst. Henri Poincaré, Prob. Stat. 56 (2020) 637-666.

[14] P. Collet, S. Martinez and J.S. Martin, Quasi-stationary distributions. Markov chains, diffusions and dynamical systems (2013).

[15] P. Del Moral and L. Miclo, Particle approximations of Lyapunov exponents connected to Schrödinger operators and FeynmanKac semigroups. ESAIM: PS $\mathbf{7}$ (2003) 171-208.

[16] P. Del Moral and L. Miclo, A Moran particle system approximation of Feynman-Kac formulae. Stoch. Process. Appl. 86 (2000) 193-216.

[17] P. Del Moral and L. Miclo, On convergence of chains with occupational self-interactions. Proc. Royal Soc. Lond. Ser. A: Math. Phys. Eng. Sci. 460 (2004) 325-346.

[18] P. Del Moral and L. Miclo, Self-interacting Markov chains. Stoch. Anal. Appl. 24 (2006) 615-660.

[19] B. Delyon, Stochastic approximation with decreasing gain: convergence and asymptotic theory. Tech. report, IRISA (2000), Publication interne 952.

[20] W.H. Fleming and M. Viot, Some measure-valued Markov processes in population genetics theory. Indiana Univ. Math. J. 28 (1979) 817-843.

[21] G. Fort, Central limit theorems for stochastic approximation with controlled Markov chain dynamics. ESAIM: PS 19 (2013).

[22] P. Groisman and M. Jonckheere, Simulation of quasi-stationary distributions on countable spaces. Markov Process. Related Fields 19 (2012).

[23] P. Hall and C. Heyde, Martingale Limit Theory and its Applications. Academic Press (1980).

[24] R.A. Horn and C.R. Johnson, Topics in Matrix Analysis. Cambridge University Press, New York, New York (1991).

[25] J. Kiefer and J. Wolfowitz, Stochastic estimation of the maximum of a regression function. Ann. Math. Stat. 23 (1952) $462-466$.

[26] A.N. Kolmogorov, On the solution of a problem in biology. Izv. NII Matem. Mekh. Tomskogo Univ. 2 (1938) 7-12.

[27] H. Kushner and G. George Yin, Vol. 35 of Stochastic Approximation and Recursive Algorithms and Applications. Springer Science \& Business Media (2003).

[28] T. Lelievre, L. Pillaud-Vivien and J. Reygner, Central limit theorem for stationary Fleming-Viot particle systems in finite spaces. ALEA 15 (2018) 1163-1182.

[29] S. Méléard and D. Villemonais, Quasi-stationary distributions and population processes. Prob. Surv. 9 (2012) $340-410$.

[30] P.K. Pollett, Quasi-stationary distributions: a bibliography. http://www.maths.uq.edu.au/pkp/papers/qsds/qsds.pdf (2008).

[31] H. Robbins and S. Monro, A stochastic approximation method. Ann. Math. Stat. (1951) 400-407.

[32] B.A. Sevast'yanov, The theory of branching random processes. Uspekhi Mat. Nauk. 6 (1951) 47-99.

[33] N.G. Van Kampen, Stochastic Processes in Physics and Chemistry. Elsevier, North-Holland, Amsterdam (1992).

[34] D. Villemonais, General approximation method for the distribution of Markov processes conditioned not to be killed. ESAIM: $P S 18$ (2014) 441-467.

[35] A.Q. Wang, G.O. Roberts and D. Steinsaltz, An approximation scheme for quasi-stationary distributions of killed diffusions. Stoch. Process. Appl. 130 (2020) 3193-3219.

[36] A.M. Yaglom, Certain limit theorems of the theory of branching processes. Dokl. Acad. Nauk. SSSR 56 (1947) $795-798$.

\section{Subscribe to Open (S2O)}

\section{A fair and sustainable open access model}

This journal is currently published in open access under a Subscribe-to-Open model (S2O). S2O is a transformative model that aims to move subscription journals to open access. Open access is the free, immediate, online availability of research articles combined with the rights to use these articles fully in the digital environment. We are thankful to our subscribers and sponsors for making it possible to publish this journal in open access, free of charge for authors.

\section{Please help to maintain this journal in open access!}

Check that your library subscribes to the journal, or make a personal donation to the S2O programme, by contacting subscribers@edpsciences.org

More information, including a list of sponsors and a financial transparency report, available at: https://www.edpsciences.org/en/maths-s2o-programme 